\title{
Minimally Empirical Double Hybrid Functionals Trained Against the GMTKN55 Database: revDSD-PBEP86-D4, revDOD-PBE-D4, and DOD-SCAN-D4
}

Golokesh Santra, Nitai Sylvetsky, Gershom Martin

Submitted date: 22/05/2019 - Posted date: 22/05/2019

Licence: CC BY-NC-ND 4.0

Citation information: Santra, Golokesh; Sylvetsky, Nitai; Martin, Gershom (2019): Minimally Empirical Double Hybrid Functionals Trained Against the GMTKN55 Database: revDSD-PBEP86-D4, revDOD-PBE-D4, and DOD-SCAN-D4. ChemRxiv. Preprint.

We present a family of minimally empirical double-hybrid DFT functionals parametrized against the very large and diverse GMTKN55 benchmark. The very recently proposed wB97M(2) empirical double hybrid (with 16 empirical parameters) has the lowest WTMAD2 (weighted mean absolute deviation over GMTKN55) ever reported at $2.19 \mathrm{kcal} / \mathrm{mol}$. However, our xrevDSD-PBEP86-D4 functional reaches a statistically equivalent WTMAD2=2.22 kcal/mol, using just a handful of empirical parameters, and the xrevDOD-PBEP86-D4 functional reaches $2.25 \mathrm{kcal} / \mathrm{mol}$ with just opposite-spin MP2 correlation, making it amenable to reduced-scaling algorithms. In general, the D4 empirical dispersion correction is clearly superior to D3BJ. If one eschews dispersion corrections of any kind, noDispSD-SCAN offers a viable alternative. Parametrization over the entire GMTKN55 dataset yields substantial improvement over the small training set previously employed in the DSD papers.

File list (2)

revDSD-PBEP86-D4 revision resubmission.pdf (762.64 KiB)

view on ChemRxiv - download file 


\title{
Minimally Empirical Double Hybrid Functionals Trained Against the GMTKN55 Database: revDSD-PBEP86-D4, revDOD-PBE-D4, and DOD-SCAN-D4
}

\author{
Golokesh Santra, Nitai Sylvetsky, and Jan M.L. Martin \\ Department of Organic Chemistry, Weizmann Institute of Science, 7610001 Rehovot, Israel. \\ Email: gershom@weizmann.ac.il
}

\section{Abstract}

We present a family of minimally empirical double-hybrid DFT functionals parametrized against the very large and diverse GMTKN55 benchmark. The very recently proposed $\omega \mathrm{B} 97 \mathrm{M}(2)$ empirical double hybrid (with 16 adjustable parameters) has the lowest WTMAD2 (weighted mean absolute deviation over GMTKN55) ever reported at $2.19 \mathrm{kcal} / \mathrm{mol}$. However, refits of the DSD-BLYP and DSD-PBEP86 spin-component-scaled, dispersion-corrected double hybrids can achieve WTMAD2 values as low as 2.33 with the very recent D4 dispersion correction, $(2.42$ $\mathrm{kcal} / \mathrm{mol}$ with the D3(BJ) dispersion term) using just a handful of adjustable parameters. If we use full DFT correlation in the initial orbital evaluation, the xrevDSD-PBEP86-D4 functional reaches WTMAD2=2.23 kcal/mol, statistically indistinguishable from $\omega \mathrm{B} 97 \mathrm{M}(2)$ but using just four non-arbitrary adjustable parameters (and three semi-arbitrary ones). The changes from the original DSD parametrizations are primarily due to reaction and isomerization energies for large systems, which were undersampled in the original parametrization set. With the new parametrization, same-spin correlation can be eliminated at minimal cost in performance, which permits revDOD-PBEP86-D4 and revDOD-PBE-D4 functionals that scale as $\mathrm{N}^{4}$ or even $\mathrm{N}^{3}$ with the size of the system. Dependence of WTMAD2 for DSD functionals on the percentage of HF exchange is roughly quadratic; it is sufficiently weak that any reasonable value in the $64 \%$ to $72 \%$ range can be chosen semi-arbitrarily. DSD-SCAN and DOD-SCAN double hybrids involving the SCAN non-empirical meta-GGA as the semi-local component have also been considered, and offer a good alternative if one wishes to eliminate either the empirical dispersion correction or the same-spin correlation component. noDispSD-SCAN66 achieves WTMAD2=3.0 $\mathrm{kcal} / \mathrm{mol}$, compared to $2.7 \mathrm{kcal} / \mathrm{mol}$ for DOD-SCAN66-D4. However, the best performance without dispersion corrections $(\mathrm{WTMAD} 2=2.8 \mathrm{kcal} / \mathrm{mol}$ ) is reached by rev $\omega \mathrm{B} 97 \mathrm{X}-2$, a slight reparametrization of the Chai-Head-Gordon range-separated double hybrid. Finally, in the context of double-hybrid functionals, the very recent D4 dispersion correction is clearly superior over D3(BJ).

\section{Introduction}

Large and chemically diverse standardized reference datasets play a crucial role in the validation of new approximate computational chemistry methods (not just density functional methods, but also semi-empirical molecular orbital methods, e.g., ${ }^{1}$, composite wavefunction ab initio schemes, ${ }^{2-6}$ and machine learning-assisted approaches ${ }^{7}$ ).

If these approaches are devoid of empirical parameters (such as the "non-empirical" DFT functionals PBE, ${ }^{8}$ TPSS, ${ }^{9}$ and SCAN, ${ }^{10}$ as well as the ccCA, ${ }^{11} \mathrm{~W} 1,5,12$ and $\mathrm{W} 1-\mathrm{F} 12^{6}$ approaches), then the purpose of these datasets is only validation. If the methods include empirical parameters, 
however (such as in "empirical" DFT functionals, e.g., B97-1, ${ }^{13} \mathrm{HCTH},{ }^{14} \mathrm{BMK},{ }^{15} \mathrm{M} 06,{ }^{16}$ MN15, ${ }^{17}$ and many others), then such datasets take on the additional role of "training sets" or parametrization sets. In the earliest days, small sets of experimental data were used for this purpose, e.g. for B3LYP ${ }^{18}$ and EDF-1; ${ }^{19}$ as the practical limitations of this approach became apparent, Handy ${ }^{14,20}$ pioneered the use of high-level wavefunction ab initio data for the same purpose. This approach has perhaps been taken furthest by the Head-Gordon group in their combinatorially optimized $\omega \mathrm{B} 97 \mathrm{X}-\mathrm{V},{ }^{21} \mathrm{~B} 97 \mathrm{M}-\mathrm{V},{ }^{22} \omega \mathrm{B} 97 \mathrm{M}-\mathrm{V},{ }^{23}$ and $\omega \mathrm{B} 97 \mathrm{M}(2)^{24}$ functionals.

In Perdew's "Jacob's Ladder" metaphor, ${ }^{25}$ Hartree theory represents the "Earthly vale of tears" and the introduction of each new type of information one more rung on the "Jacob's Ladder" ascending to the Heaven of chemical accuracy. The first rung corresponds to the local density approximation, where the XC (exchange-correlation) functional only depends on the density $\rho$. The reduced density gradient is introduced on the second rung, leading to the various GGA (generalized gradient approximation) functionals. The introduction of the Laplacian (or the kinetic energy density, which contains similar information) creates the third rung, the metaGGAs. The fourth rung introduces dependence on the occupied orbitals: ${ }^{26}$ the most important special case are the different types of hybrid functionals. Finally, the fifth rung corresponds to dependence on virtual orbitals, such as double-hybrid functionals. The term "doubly hybrid" was first coined to denote the linear combination of GGA correlation and MP2 correlation from HF orbitals, ${ }^{27}$ but since the landmark paper of Grimme, ${ }^{28}$ the term "double hybrid" has come to refer exclusively to the admixture of (meta)GGA DFT correlation with GLPT2 (2 ${ }^{\text {nd }}-$ order GörlingLevy $^{29}$ perturbation theory) correlation from hybrid (meta)GGA DFT orbitals.

In the first step of a double-hybrid calculation, a Kohn-Sham calculation is carried out for a given semilocal exchange-correlation (XC) functional with a fraction $\mathrm{c}^{\prime}{ }_{\mathrm{x}}$ of Hartree-Fock exchange and $\left(1-\mathrm{c}_{\mathrm{x}}^{\prime}\right)$ of XC exchange, plus XC correlation damped by a factor $\mathrm{c}^{\prime} \mathrm{C}, \mathrm{DFA}$. With the converged Kohn-Sham orbitals at the end, the total energy is then evaluated in the second step as:

$$
E=E_{N 1 e}+c_{x} E_{x, H F}+\left(1-c_{x}\right) E_{x, X C}+c_{c, X C} E_{c, X C}+c_{2 a b} E_{2 a b}+c_{2 s s} E_{2 s s}+E_{d i s p}
$$

where $E_{\mathrm{N} 1 \mathrm{e}}$ stands for the nuclear repulsion and one-electron energy term; $\mathrm{E}_{\mathrm{x}, \mathrm{HF}}$ is the Hartree-Fock exchange energy and $c_{x}$ the fraction of Hartree-Fock-like exchange energy; $\mathrm{E}_{\mathrm{x}, \mathrm{XC}}$ and $\mathrm{E}_{\mathrm{c}, \mathrm{XC}}$ are the exchange and correlation energies, respectively, for the given semilocal XC functional with the converged density from the first step, and $\mathrm{c}_{\mathrm{c}, \mathrm{XC}}$ the fraction of DFT correlation energy; $\mathrm{E}_{2 \mathrm{ab}}$ and $\mathrm{E}_{2 \mathrm{ss}}$ are the opposite-spin and same-spin MP2-like energies obtained in the basis of Kohn-Sham like orbitals from the first step, and $c_{2 a b}$ and $c_{2 s s}$ are the linear coefficients for the same; and, finally, $\mathrm{E}_{\text {disp }}$ is the dispersion energy obtained from a given empirical dispersion model (e.g., D2, ${ }^{30} \mathrm{D} 3(0),{ }^{31} \mathrm{D} 3(\mathrm{BJ}),{ }^{31,32}$ or very recently, $\left.{ }^{33,34} \mathrm{D} 4\right)$ or nonlocal dispersion functional (such as $\mathrm{VV} 10^{35}$ ), which can itself be dependent on scaling or shaping parameters. In much of the present work, $\mathrm{E}_{\mathrm{disp}}$ is proportional to a pre-factor $\mathrm{s} 6$.

In the original Grimme approach, ${ }^{28} \mathrm{c}_{\mathrm{x}}{ }_{\mathrm{x}}=\mathrm{c}_{\mathrm{x}}, \mathrm{c}_{\mathrm{c}, \mathrm{XC}}=\mathrm{c}_{\mathrm{c}, \mathrm{XC}}$, and $\mathrm{c}_{2 \mathrm{ab}}=\mathrm{c}_{2 \mathrm{ss}}$; the latter constraint in practice restricts ${ }^{36,37}$ the choice of correlation functionals to LYP. In the DSD (dispersion-corrected, spin-component-scaled double hybrid ${ }^{38}$ ) functionals of Kozuch and Martin, the constraint $\mathrm{c}_{2 \mathrm{ab}}=\mathrm{c}_{2 \mathrm{ss}}$ is relaxed: this was found $\mathrm{d}^{36,37}$ to enable a broader variety of exchange-correlation functionals, with DSD-PBEP86 the best performer at that point. In the GMTKN55 benchmark paper, the best two performers were DSD-PBEP86 and DSD-BLYP, followed by B2GP-PLYP. ${ }^{39}$ 
In the XYG3 approach of Goddard, Xu, and coworkers, ${ }^{40} \mathrm{c}_{\mathrm{c}, \mathrm{XC}}=1 \neq \mathrm{c}_{\mathrm{c}, \mathrm{XC}}$ and $\mathrm{c}_{\mathrm{X}}{ }_{\mathrm{x}}$ may differ from $c_{x}$ : the implication of this choice have been discussed at length elsewhere. ${ }^{41-43}$ With two exceptions (namely, $\omega \mathrm{B} 97 \mathrm{M}(2)^{24}$ and xrevDSD-PBEP86-D4, see below), functionals of this form are not discussed in this paper. The special case $c^{\prime}{ }_{x}=c_{x}$ in a DSD context has been denoted $\mathrm{xDSD}^{42}$

Double hybrid DFT has been reviewed by Goerigk and Grimme, ${ }^{44}$ by Sancho-Garcia and Adamo, ${ }^{45}$ and by $\mathrm{Xu}$ and coworkers. ${ }^{40,46}$ An extensive comparative study between nonempirical ${ }^{47}$ and semiempirical (e.g., Refs. ${ }^{28,36,37,39,48-50}$ ) double hybrids was recently made by Goerigk and coworkers ${ }^{51}$, who found semiempirical functionals (at present) to be more accurate and more robust. For some recent perspectives on "the functional zoo" (Perdew's term), see, e.g., Refs. ${ }^{52-54}$

Perhaps the two most extensive and chemically diverse validation datasets around are GMTKN55 (General Main-group Thermochemistry, Kinetics, and Noncovalent interactions, 55 problem subsets) of Goerigk, Grimme, and coworkers ${ }^{55}$ — which has about 1,500 nonredundant reaction energies and barrier heights - and the even larger MGCDB84 (Main Group Chemistry DataBase, 84 problem subsets) of Mardirossian and Head-Gordon, ${ }^{53}$ which has close to 5,000 such nonredundant energy differences. These databases themselves incorporate and extend upon earlier work by these authors themselves (e.g., ${ }^{41,56}$ ), by the Minnesota group (Refs. ${ }^{54,57}$ and references therein), by the Hobza group (particularly for noncovalent interactions ${ }^{58-61}$ ) and by the present research team (e.g., ${ }^{39,62-73}$.)

Such large and unwieldy reference datasets have themselves inspired the statistical search for representative subsamples that would recover most of the variation in the underlying dataset yet be much easier to handle, particularly as training sets where re-evaluation of the whole dataset at every parameter combination would quickly become unwieldy. To the authors' knowledge, the first such study was Ref. ${ }^{74}$; the two most recent ones are MG8 by Chan ${ }^{75}$, a 60 reaction subset of MGCDB84 obtained through lasso regularization, and "Diet-GMTKN55" by Gould $^{76}$, the latter of which proposes 30-, 100-, and 150-reaction "Diet" versions of GMTKN55. Aside from "rapid prototyping", these could in principle serve as training sets for empirical functionals, with the full dataset then used for validation purposes.

Our explorations on the suitability of such reduced training sets for functional development will be discussed elsewhere. In the present paper, we focus instead on the full GMTKN55 benchmark as being sufficiently large and chemically varied that parametrization and validation against it is largely immune to sample bias. To the best of our knowledge, the present work is the first paper in which the full GMTKN55 dataset is used as a training set for DFT functionals, although we are also validating some new functionals not covered in the original GMTKN55 paper (for technical reasons).

We will show below that:

(a) the most accurate functional that does not entail the fifth rung of Perdew's "Jacob's Ladder" 25 is the combinatorially optimized, range-separated, hybrid meta-GGA $\omega$ B97M$\mathrm{V}$, again by the Berkeley group ${ }^{23}$

(b) if the search is widened to fifth-rung options, the combinatorially optimized, rangeseparated, double hybrid $\omega \mathrm{B} 97 \mathrm{M}(2)$ by Mardirossian and Head-Gordon ${ }^{24}$ is at present the most accurate functional available for general main-group chemistry;

(c) this having been said, reparametrized versions of DSD-BLYP-D3(BJ) ${ }^{38}$ and DODPBEP86-D3(BJ) ${ }^{36,37}$ fitted to GMTKN55 come quite close in performance with just onethird the number of empirical parameters; 
(d) replacing the $\mathrm{D} 3(\mathrm{BJ})$ dispersion correction by the more modern, partial-charge dependent D4 model significantly enhances performance;

(e) the xrevDSD-PBEP86-D4 model affords a statistically equivalent WTMAD2 to $\omega \mathrm{B} 97 \mathrm{M}(2)$, as does its xrevDOD-PBEP86-D4 variant, which is amenable to reducedscaling MP2 implementations;

(f) if one eschews empirical dispersion corrections, then the noDispSD-SCAN63

functional proposed in the present work offers the best performance;

(g) while performance over GMTKN55 is markedly improved from the original versus

the refitted DSD functionals, performance for small-molecule atomization energies and barrier heights is barely affected - the improvements are seen in large-molecule isomerization and reaction energies where there is an important dispersion component; (h) this presents a cautionary tale about "overfitting" to small and insufficiently diverse reference samples.

\section{Computational Methods}

\section{Reference data}

As our primary parametrization and validation set, we used the comprehensive GMTKN55 benchmark ${ }^{55}$ of Goerigk, Grimme, and coworkers. This set, itself a further expansion and update of earlier GMTKN ${ }^{56}$ and GMTKN30 $0^{41}$ datasets, is a composite of fifty-five chemical problem types, ranging from small-molecule thermochemistry and barrier heights to large-molecule isomerization energies, noncovalent interactions, conformational equilibria, self-interaction errors, heavy p-block chemistry, ion chemistry,... intending to cover all aspects of main-group chemistry. Their reference data had been compiled from high-level ab initio benchmark studies in the literature, supplemented by some new benchmark calculations of their own. A detailed breakdown of the 55 subsets (and full source information for the original reference data) can be found in Table S1 in the electronic supporting information (ESI): suffice to say a full evaluation entails 2,459 electronic structure calculations for 1,499 chemical energy differences.

The reference geometries, charge and multiplicity information, and reference data were extracted from the ACCDB database of Morgante and Peverati ${ }^{77}$. While initial runs were made with the help of the Snakemake ${ }^{78}$ workflows defined as part of ACCDB, once we had a full set of input files we elected to use our own scripting. Data analysis was carried out using a Fortran program developed in-house and available on request from the authors.

The primary metric and "objective function" employed is the WTMAD2 (weighted mean absolute deviation, type 2) as defined by Goerigk et al. ${ }^{55}$ It seeks to compensate both for the different energy scales various properties are on and for the different sizes of the various subsets:

$$
\text { WTMAD2 }=\frac{1}{\sum_{i}^{55} N_{i}} \cdot \sum_{i}^{55} N_{i} \cdot \frac{56.84 \mathrm{kcal} / \mathrm{mol}}{{\overline{|\Delta E|_{i}}}_{i}} \cdot \mathrm{MAD}_{i}
$$

in which $|\Delta \mathrm{E}|_{i}$ is the mean absolute value of all the reference energies for subset $i, \mathrm{~N}_{i}$ the number of systems in the subset, and $\mathrm{MAD}_{i}$ represents the mean absolute difference between calculated and reference reaction energies for subset $i$. We note that MAD is a more "robust statistic" than the root mean square deviation, in the statistical sense that MAD is less prone to 
hypersensitivity to one or a few "outlier" points than the RMSD (root mean square deviation), even as the latter is more useful for spotting "troublemakers" for the exact same reason.

\section{Electronic structure details}

Reference geometries were used "as is" and not optimized further. The Weigend-Ahlrichs def2QZVPP basis set ${ }^{80}$ was used for most systems, except for the subsets WATER27, RG18, IL16, G21EA, and AHB21 where we used the diffuse-function augmented def2-QZVPPD instead, ${ }^{81}$ and the large-molecule isomerization subsets C60ISO and UPU23, where we compromised on the def2-TZVPP basis set for reasons of computational cost. ${ }^{80}$

All calculations were carried out using Q-CHEM 5.1.1 ${ }^{82}$ running on the ChemFarm HPC cluster of the Weizmann Institute Faculty of Chemistry. For GGAs and double hybrids derived from them, initial calculations employed the SG-2 integration grid, ${ }^{83}$ which is a pruned $(75,302)$ grid roughly comparable to the (Grid=Fine) in Gaussian; the notation stands for the direct product of a 75-point Euler-Maclaurin radial quadrature ${ }^{84,85}$ and a 302-point Lebedev angular grid (see Ref. ${ }^{86}$ and references therein). For meta-GGAs and double hybrids derived from them, we employed the larger SG-3 grid, which is a pruned $(99,590)$ grid roughly comparable to (Grid=UltraFine) in Gaussian; ultimately, we also recalculated the GGAs and double-hybrid GGAs from them. As can be seen in the Electronic supporting information (Table S12), the switch to an SG-3 grid makes a major difference for the RG18 rare gas complexes subset, and a minor but nontrivial one for the anionic subsets. For the SCAN (strongly constrained and appropriately normed ${ }^{10}$ ) meta-GGA — which exhibits a well-known ${ }^{87}$ integration grid hypersensitivity - after some experimentation we decided on an unpruned $(150,590)$ grid, which for a subset we checked for convergence against an even larger $(200,974)$ grid.

The combinatorially optimized range-separated hybrid (RSH) GGA $\omega B 97 \mathrm{X}-\mathrm{V},{ }^{21}$ its RSH meta-GGA successor $\omega B$ 97M-V ${ }^{23}$ (and its meta-GGA ancestor B97M-V ${ }^{22}$ ), and finally its very recent double hybrid spinoff $\omega \mathrm{B} 97 \mathrm{M}(2)$ were evaluated using their respective implementations in Q-CHEM, ${ }^{24}$ as was the older range-separated double hybrid GGA $\omega$ B97X2(TQ) of Chai and Head-Gordon. ${ }^{88}$

In the GMTKN55 paper, all electrons were correlated in the MP2-like steps of the double hybrids. While the def2-QZVPP basis set used there and in the present work is not really suitable for core-valence correlation, we have calculated statistics both with and without frozen innershell orbitals. In both cases, however, we have elected to correlate the subvalence electrons of the metal and metalloid atoms in subsets MB16-43, HEAVY28, HEAVYSB11, ALK8, and ALKBDE10 sets, as the core-valence gaps with default frozen orbital settings are smaller than 1 hartree. Indeed, for alkali and alkali earth oxides and halides, subvalence (n-1)p orbitals may otherwise intrude into the valence band, and thus result in nonsensical dissociation energies with standard frozen-core settings, as discussed at length in e.g., Refs. ${ }^{89,90}$

Initially we unfroze all subvalence electrons in HEAVY28 and HAL59. (The importance of subvalence correlation for halogen-bonded species has been shown previously ${ }^{63}$ for the $\mathrm{X} 40 \times 10$ dataset $^{60}$ ) However, while the added binding energy from (1s) orbitals of CNOF elements, on the order of $0.3 \mathrm{kcal} / \mathrm{mol}$, might be negligible compared to covalent bond dissociation energies, it represents a nontrivial percentage of the small reaction energies in these sets, which owing to the weighting of subsets by inverse mean absolute reaction energies in WTMAD2 causes an increase of up to $0.15 \mathrm{kcal} / \mathrm{mol}$. (As shown in Ref. ${ }^{38}$, double hybrid parameters optimized with core correlation have slightly different coefficients to compensate for 
the extra correlation energy.) We have hence, in these systems, manually unfrozen the (n-1)spd orbitals of the heavy elements but not any deeper core orbitals such as the (1s) of first-row elements.

For the sake of comparison and completeness, we also evaluated WTMAD2 in the exact same manner for some additional functionals on rungs $2-4$. They were the GGAs PBE, ${ }^{8}$ revPBE, ${ }^{91}$ and B97-D3(BJ); ${ }^{31}$ the meta-GGAs TPSS, ${ }^{9}$ revTPSS, ${ }^{92} \mathrm{SCAN},{ }^{10}$ the global hybrid GGAs B3LYP, ${ }^{18,93}$ PBE0,${ }^{94}$ and its analog revPBE0 $;{ }^{94}$ the global hybrid meta-GGAs PW6B95, ${ }^{95}$ M06, ${ }^{96}$ M06-2X, ${ }^{96}$ SCAN0, ${ }^{97}$ and MN15. ${ }^{17}$ With the exception of M06 and M06-2X, all these functionals were used in conjunction with $\mathrm{D} 3(\mathrm{BJ})$ corrections: parameters published in Ref. ${ }^{55}$ were used throughout. For M06, D3(0) was used instead as recommended in Ref. ${ }^{41}$, where it was found that D3(BJ) parameter optimizations for M06 and M06-2X diverge; for M06, we employed D3(0) instead as per their recommendation, while for M06-2X, we found that D3(0) in fact yields slightly worse WTMAD2 than the uncorrected functional. While the difference is statistically insignificant, we used the uncorrected M06-2X instead according to lex parsimoniae (a.k.a. Occam's Razor).

\section{Optimization details}

The BOBYQA (Bound Optimization BY Quadratic Approximation) derivative-free constrained optimizer $^{98}$ by Powell was used as the core of a computer program and collection of shell scripts developed in-house.

A DSD double hybrid, if fully optimized, has six empirical parameters:

(a) the fraction of HF exchange $\mathrm{c}_{\mathrm{X}, \mathrm{HF}}$. (The fraction of semilocal DFT exchange is always $\mathrm{c}_{\mathrm{X}, \mathrm{DFT}}=1-\mathrm{c}_{\mathrm{X}, \mathrm{HF}}$.)

(b) the fraction of semilocal DFT correlation $\mathrm{c}_{\mathrm{C}, \mathrm{DFT}}$;

(c) the fraction of opposite-spin $2^{\text {nd }}$-order GLPT correlation energy $c_{2 a b}$;

(d) the fraction of same-spin $2^{\text {nd }}$-order GLPT correlation energy $\mathrm{c}_{2 \mathrm{ss}}=\mathrm{c}_{2 \mathrm{aa}+\mathrm{bb}}$;

(e) the prefactor $\mathrm{s}_{6}$ for the $\mathrm{D} 3(\mathrm{BJ})$ empirical dispersion correction ${ }^{31,32,99}$;

(f) the length scale parameter $\mathrm{a}_{2}$ for the D3(BJ) damping function. (As in Refs. ${ }^{36,37}$, we are setting $\mathrm{a}_{1}=0$; we are also setting $\mathrm{s}_{8}=0$ as in Refs. ${ }^{36,37}$, and in the SCAN-D3(BJ) ${ }^{87}$ paper.) For a given pair of values for $(a, b)$, it is possible to obtain the optimal group of (c-f) parameters without re-evaluating any electronic structure calculations, simply by extracting individual energy components from the electronic structure calculations, evaluating total energies and hence WTMAD2 for a given combination of $\left\{\mathrm{c}_{2 \mathrm{ab}}, \mathrm{c}_{2 \mathrm{ss}}, \mathrm{s}_{6}, \mathrm{a}_{2}\right\}$, and minimizing WTMAD2 with respect to these four parameters using BOBYQA. This could then constitute an inner "microiteration"

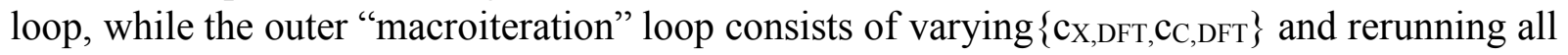
2,459 calculations with the new parameters. We considered, however, placing one or both variables in the microiteration loop, with the optimum values from the microiterations to be used in the macroiterations, and so forth until "self-consistency" has been reached. While the coupling between (a) and (c,d) proved to strong for this to be viable for $\mathrm{c}_{X, D F T}$,we found that for a fixed value of $c_{X, D F T}$, convergence of $c_{C, D F T}$ to two decimal places or better typically does not require more than two macroiterations. Hence, we have adopted the practice of microiterating $\left\{\mathrm{c}_{\mathrm{C}, \mathrm{DFT}}, \mathrm{c}_{2 \mathrm{ab}}, \mathrm{c}_{2 \mathrm{ss}}, \mathrm{s}_{6}, \mathrm{a}_{2}\right\}$ at every macroiteration by means of BOBYQA.

The D3(BJ) corrections were computed for a finely spaced grid in a 2 using the standalone DFTD3 program by Grimme and coworkers (https://www.chemie.uni-bonn.de/pctc/mullikencenter/software/dft-d3/). Values for intermediate $\mathrm{a}_{2}$ were obtained by interpolation. We found, however (see below) that if $\mathrm{s}_{6}$ is part of the microiterations, then the WTMAD2 surface is 
sufficiently flat in $a_{2}$ that fixing $a_{2}$ at semi-arbitrary values both stabilizes the optimization and has negligible effect on the final WTMAD2.

For a DOD double hybrid, $\mathrm{c}_{2 \mathrm{ss}}=0$, leaving just four parameters $\left.\mathrm{c}_{\mathrm{C}, \mathrm{DFT}}, \mathrm{c}_{2 \mathrm{ab}}, \mathrm{s}_{6}, \mathrm{a}_{2}\right\}$ for the microiterations, while for a DSD-noDisp, $\mathrm{s}_{6}=0$ and $\mathrm{a}_{2}$ is irrelevant, leaving just three parameters $\left\{\mathrm{c}_{\mathrm{C}, \mathrm{DFT}}, \mathrm{c}_{2 \mathrm{ab}}, \mathrm{c}_{2 \mathrm{ss}}\right\}$ in the inner loop.

\section{Results and Discussion}

WTMAD2 performance metrics over the GMTKN55 dataset are given in Table 1. Results for a large number of GGA, meta-GGAs, and hybrid functionals have been given in the GMTKN55 paper $^{55}$ and will not be repeated here. We have repeated the calculations for B3LYP, PBE0, and $\omega \mathrm{B} 97 \mathrm{X}-\mathrm{V}$ as a sanity check; in addition, we have evaluated the B97M-V and $\omega \mathrm{B} 97 \mathrm{M}-\mathrm{V}$ functionals, which were not considered in the original GMTKN55 paper but were covered in a recent follow-up study by Najibi and Goerigk )NG), ${ }^{100}$ and finally the $\omega \mathrm{B} 97 \mathrm{M}(2)$ double hybrid, for which no GMTKN55 statistics have yet been reported.

As very recently found by $\mathrm{NG}$, the switch from a combinatorially optimized rangeseparated hybrid GGA (coRSH GGA) in $\omega$ B97X-V to a coRSH meta-GGA in $\omega$ B97M-V represents a clear improvement over $\omega \mathrm{B} 97 \mathrm{X}-\mathrm{V}$; we find that WTMAD2 goes down from 3.96 $\mathrm{kcal} / \mathrm{mol}$ to $3.29 \mathrm{kcal} / \mathrm{mol}$. This latter figure is the lowest WTMAD2 reported thus far for a hybrid functional: $\omega \mathrm{B} 97 \mathrm{X}-\mathrm{V}$ was the previous best contender in the original GMTKN55 paper. Breakdown by components (Table S11) reveals conspicuous accuracy gains for the pericyclic reaction barriers (BHPERI), for bond separation reactions of saturated hydrocarbons (BSR36), and large system reaction energies more generally. By way of data reduction, we may in fact consider the sums of WTMAD2 contributions for each of the five major subcategories in GMTKN55: Thermochemistry, Intermolecular interactions, Conformers, Barrier heights, and Reaction energies for large systems.

Like NG earlier, we find B97M-V and $\omega$ B97M-V to be "best in class" on Rungs 3 and 4, respectively, of the Jacob's Ladder.

It then becomes apparent (Table 2) that the chief gain for $\omega \mathrm{B} 97 \mathrm{M}-\mathrm{V}$ over $\omega \mathrm{B} 97 \mathrm{X}-\mathrm{V}$ is in fact for thermochemistry and large system reaction energies. There is a small improvement for barrier heights, but no change for intermolecular interactions and in fact a slight deterioration for conformers; upon further inspection, the latter can be attributed primarily to the tri-and tetrapeptide conformers (PCONF21). The already excellent statistic for $\omega \mathrm{B} 97 \mathrm{M}-\mathrm{V}$ can be brought down even further to $2.19 \mathrm{kcal} / \mathrm{mol}$ with the coRSH mGGA double hybrid $\omega \mathrm{B} 97 \mathrm{M}(2)$. This improvement is actually seen for all subcategories across the board. The WTMAD2 value for $\omega \mathrm{B} 97 \mathrm{M}(2)$ is, by some distance, the lowest reported for any functional thus far: the best three performers from the original GMTKN55 paper, ${ }^{55}$ DSD-BLYP-D3(0), DSD-BLYP-D3(BJ), and DSD-PBEP86-D3(BJ), clocked in at 3.00, 3.08, and $3.14 \mathrm{kcal} / \mathrm{mol}$, respectively, while in the followup paper, NG found DSD-PBEP86-NL to be even better at $2.84 \mathrm{kcal} / \mathrm{mol}$. In that same paper, the runners-up were $\omega$ B97X-2(TQ)-D3(BJ) and DSD-BLYP-NL at 2.97 and 3.05 $\mathrm{kcal} / \mathrm{mol}$, respectively. In the present paper, we found 3.06 for $\omega \mathrm{B} 97 \mathrm{X}-2(\mathrm{TQ})-\mathrm{D} 3(\mathrm{BJ})$ : detailed analysis revealed that essentially the whole difference can be attributed to the larger def2QZVPPD basis set used in RG18, which has a disproportionately large weight factor owing to the small average reaction energy in the denominator. (Note that using grids smaller than SG-3 for RG18 causes errors in WTMAD as large as the first decimal place!) 
Table1: WTMAD2 values (kcal/mol) for various functionals using the full GMTKN55 database.

\begin{tabular}{|c|c|c|c|c|c|}
\hline \multirow{3}{*}{ Functionals } & \multicolumn{5}{|c|}{ WTMAD2(kcal/mol) } \\
\hline & \multirow[b]{2}{*}{ Standard/Original } & \multicolumn{4}{|c|}{ With Refitted $a_{2}$} \\
\hline & & DSD & $\begin{array}{c}\text { DOD } \\
\left(c_{2 s s}=0\right)\end{array}$ & noDispSD $\left(s_{6}=0\right)$ & $\begin{array}{l}\text { noDispOD } \\
\left(c_{2 s s}==_{6}=0\right)\end{array}$ \\
\hline PBE-D3(BJ) & 10.44 & & & & \\
\hline TPSS-D3(BJ) & 9.14 & & & & \\
\hline B97-D3(BJ) & 8.61 & & & & \\
\hline revTPSS-D3(BJ) & 8.42 & & & & \\
\hline revPBE-D3(BJ) & 8.34 & & & & \\
\hline SCAN-D3(BJ) & 7.94 & & & & \\
\hline M06-D3(0) & 7.75 & & & & \\
\hline SCAN0 & 7.69 & & & & \\
\hline PBE0-D3(BJ) & 6.55 & & & & \\
\hline B3LYP-D3(BJ) & 6.50 & & & & \\
\hline B97M-rV & 6.38 & & & & \\
\hline B97M-V & 6.37 & & & & \\
\hline SCAN0-D3(BJ) $^{\mathrm{d}}$ & 6.23 & & & & \\
\hline MN15-D3(BJ) & 5.77 & & & & \\
\hline PW6B95-D3(BJ) & 5.49 & & & & \\
\hline revPBE0-D3(BJ) & 5.43 & & & & \\
\hline M062X & 4.78 & & & & \\
\hline$\omega B 97 X-V$ & 3.96 & & & & \\
\hline B2GP-PLYP & 3.33 & & & & \\
\hline$\omega \mathrm{B} 97 \mathrm{M}-\mathrm{V}$ & 3.29 & & & & \\
\hline \multicolumn{6}{|c|}{ Double hybrids without inner-shell correlation } \\
\hline$\omega \mathrm{B} 97 \mathrm{M}(2)$ & 2.19 & & & & \\
\hline$\omega \mathrm{B} 97 \mathrm{X}-2(\mathrm{TQ})-\mathrm{D} 3(\mathrm{BJ})^{88}$ & 3.06 & 2.80 & 3.71 & 2.80 & \\
\hline DSD-BLYP-D3(BJ) & 3.34 & 2.48 & 2.66 & 4.19 & 4.89 \\
\hline DSD-PBEP86-D3(BJ) & 3.10 & 2.43 & 2.45 & 3.56 & 4.07 \\
\hline DSD-PBE-D3(BJ) & 3.17 & 2.74 & 2.75 & 4.31 & \\
\hline DSD-TPSS-D3(BJ) & 3.13 & 2.95 & 3.07 & 4.27 & \\
\hline DSD-PBEB95-D3(BJ) & 3.33 & 2.85 & 2.91 & 3.53 & 3.92 \\
\hline DSD-SCAN0-2-D3(BJ) & $4.15^{\mathrm{a}}$ & 2.80 & 2.90 & 2.97 & 3.29 \\
\hline DSD-SCAN $_{74-D 3(B J)}$ & & 2.69 & 2.75 & 2.94 & 3.26 \\
\hline DSD-SCAN $_{69}-\mathrm{D} 3(\mathrm{BJ})$ & $4.53^{b}$ & 2.66 & 2.68 & 2.98 & 3.23 \\
\hline DSD-SCAN $_{66}-\mathrm{D} 3(\mathrm{BJ})$ & & 2.66 & 2.67 & 3.06 & 3.41 \\
\hline DSD-SCAN $_{63}-\mathrm{D} 3(\mathrm{BJ})$ & & 2.69 & 2.69 & 3.17 & 3.51 \\
\hline DSD-SCAN $_{58}-\mathrm{D} 3(\mathrm{BJ})$ & & 2.79 & 2.79 & 3.39 & 3.69 \\
\hline DSD-SCAN $_{55}$-D3(BJ) & & 2.89 & 2.89 & 3.58 & 3.86 \\
\hline DSD-SCAN $_{50}$-D3(BJ) & $6.11^{\mathrm{c}}$ & 3.10 & 3.10 & 3.91 & 4.14 \\
\hline \multicolumn{6}{|c|}{ Double hybrids, parameters optimized with inner-shell correlation } \\
\hline SCAN0-2 [standard] & 4.69 & & & & \\
\hline$\omega \mathrm{B} 97 \mathrm{M}(2)[$ standard] & 2.35 & & & & \\
\hline DSD-revPBEP86 & & 2.66 & & & \\
\hline DSD-BLYP-D3(BJ) & & 2.47 & 2.59 & 3.88 & \\
\hline DSD-PBEP86-D3(BJ) & & 2.46 & 2.47 & 3.35 & \\
\hline DSD-PBE-D3(BJ) & & 2.72 & 2.72 & 4.01 & \\
\hline DSD-PBEB95-D3(BJ) & & 2.87 & 2.88 & 3.38 & \\
\hline DSD-SCAN0-2-D3(BJ) & & 2.88 & 2.97 & 2.93 & \\
\hline DSD-SCAN $_{74-D 3(B J)}$ & & 2.73 & 2.77 & 2.83 & \\
\hline DSD-SCAN $_{69}-\mathrm{D} 3(\mathrm{BJ})$ & & 2.66 & 2.68 & 2.83 & \\
\hline DSD-SCAN $_{66}-\mathrm{D} 3(\mathrm{BJ})$ & & 2.65 & 2.66 & 2.88 & \\
\hline DSD-SCAN $_{63}-\mathrm{D} 3(\mathrm{BJ})$ & & 2.67 & 2.67 & 2.97 & \\
\hline DSD-SCAN $_{58}-\mathrm{D} 3(\mathrm{BJ})$ & & 2.75 & 2.75 & 3.16 & \\
\hline DSD-SCAN $_{55}-\mathrm{D} 3(\mathrm{BJ})$ & & 2.86 & 2.86 & 3.35 & \\
\hline DSD-SCAN $_{50}-\mathrm{D} 3(\mathrm{BJ})$ & & 3.07 & 3.07 & 3.68 & \\
\hline
\end{tabular}

* SCAN0-2 can also be written DSD79-SCAN; DSD69-SCAN can also be written as DSD-SCAN-QIDH; DSD55-SCAN as DSD-SCAN-CIDH; (a) SCAN0-2; ${ }^{97}$ (b) SCAN0-QIDH; ${ }^{97}$ (c) SCAN0-DH. ${ }^{97}$ (d) $\mathrm{a}_{2}=7.9042 ; \mathrm{a}_{1}=\mathrm{S}_{8}=0$ (this work) 
Table 2: WTMAD2 contribution (kcal/mol) for each of five major subcategories in cases of B97-D3(BJ), B3LYP-D3(BJ), B97M-rV, $\omega$ B97X-V, $\omega$ B97M-V \& $\omega$ B97M(2) functionals.

\begin{tabular}{|c|c|c|c|c|c|c|}
\hline \multirow{2}{*}{ Subcategories } & \multicolumn{7}{|c|}{$\Delta$ WMMAD2(kcal/mol) } \\
\cline { 2 - 7 } & B97-D3(BJ) & B3LYP-D3(BJ) & B97M-rV & $\boldsymbol{\omega B 9 7 X - V}$ & $\boldsymbol{\omega B} 97 \mathbf{M}-\mathbf{V}$ & $\boldsymbol{\omega B} 97 \mathrm{M}(2)$ \\
\hline $\begin{array}{c}\text { Intermolecular interactions } \\
\text { (Intermol) }\end{array}$ & 1.238 & 1.238 & 0.838 & 0.578 & 0.565 & 0.492 \\
\hline $\begin{array}{c}\text { Conformers/Intramolecular } \\
\text { (Conformer) }\end{array}$ & 1.542 & 1.147 & 1.810 & 0.729 & 0.897 & 0.578 \\
\hline Barrier heights (Barrier) & 1.733 & 1.141 & 1.008 & 0.561 & 0.454 & 0.258 \\
\hline Thermochemistry (Thermo) & 1.817 & 1.314 & 1.194 & 1.02 & 0.73 & 0.442 \\
\hline $\begin{array}{c}\text { Large-species reaction } \\
\text { energies (REAClarge) }\end{array}$ & 2.278 & 1.662 & 1.535 & 1.07 & 0.64 & 0.418 \\
\hline Total WTMAD2 & $\mathbf{8 . 6 0 7}$ & $\mathbf{6 . 5 0 3}$ & $\mathbf{6 . 3 8 4}$ & $\mathbf{3 . 9 5 9}$ & $\mathbf{3 . 2 8 6}$ & $\mathbf{2 . 1 8 7}$ \\
\hline
\end{tabular}

$\omega \mathrm{B} 97 \mathrm{M}(2)$ was parametrized for frozen subvalence orbitals: if correlation from such orbitals were to be included, $\mathrm{c}_{2 \mathrm{ab}}=\mathrm{c}_{2 \mathrm{ss}}$ for this functional would have to be slightly reduced to compensate. ${ }^{38}$ Indeed, when we evaluated WTMAD2 with all orbitals correlated and original parametrization, we saw an increase to $2.36 \mathrm{kcal} / \mathrm{mol}$.

Sensitivity to the percentage of Hartree-Fock exchange: DSD-SCAN as a case study The costliest parameter to vary in the refit of a DSD functional would be the percentage of Hartree-Fock exchange. As already shown in Figure 1 of Ref. ${ }^{37}$, only minimal changes in performance statistics result from varying the fraction of HF-like exchange $c_{X, H F}$ of a DSD functional within a fairly broad range - but a relatively small training set was sampled there. Presently, we will consider the case of DSD-SCAN $\mathrm{x}_{\mathrm{x}} \mathrm{D} 3(\mathrm{BJ})$ (where $\mathrm{x}$ stands for the percentage of HF exchange) in more detail: for the evaluation points, we have chosen $\mathrm{c}_{\mathrm{X}, \mathrm{HF}}=\mathrm{n}^{-1 / 3}$, where $\mathrm{n}=2,2 \frac{1}{2}, 3,3 \frac{1}{2}, 4,5,6$, and 8 . (In the "nonempirical" double hybrids of Adamo and coworkers, these choices would correspond to $\mathrm{c}_{2 \mathrm{ab}}=\mathrm{c}_{2 \mathrm{ss}}=1 / \mathrm{n}$, and $\mathrm{c}_{\mathrm{C}, \mathrm{DFT}}=1-1 / \mathrm{n}$ owing to the putative cubic dependence of the integrand. ${ }^{101,102}$ ) For the cases of $n=3,31 / 2$, and 4 , this yields values close to percentage points $0.69,0.66$, and 0.63 , respectively, and we elected to round off to these latter values.

The WTMAD2 for these trial functionals, with orbitals where $\mathrm{c}_{\mathrm{C}, \mathrm{DFT}}$ was fixed at 0.50 during the iterations (but not during the linear optimization) is depicted in Figure 1.

As can be seen there, these can be fit excellently $\left(\mathrm{R}^{2}=0.998\right.$ or better) by a simple parabola: for the DSD-SCAN-D3(BJ) curve (black), the minimum is at $\mathrm{c}_{\mathrm{X}, \mathrm{HF}}=0.683$ and the quadratic coefficient is 11.26 . The latter implies a remarkably weak sensitivity of WTMAD2 to the percentage of HF exchange: it will vary by just $0.01 \mathrm{kcal} / \mathrm{mol}$ from 0.65 to 0.71 , by 0.02 $\mathrm{kcal} / \mathrm{mol}$ over a range from 0.64 to 0.725 , and by just $0.05 \mathrm{kcal} / \mathrm{mol}$ between 0.62 and 0.750 . What this implies is that nonlinear optimization for a specific minimum $\mathrm{c}_{\mathrm{X}, \mathrm{HF}}$ becomes a somewhat academic exercise: one can choose any sensible fixed value in those ranges, such as 0.69 or 0.66 (or, if one prefers, $3^{-1 / 3}$ or $3.5^{-1 / 3}$ ). 


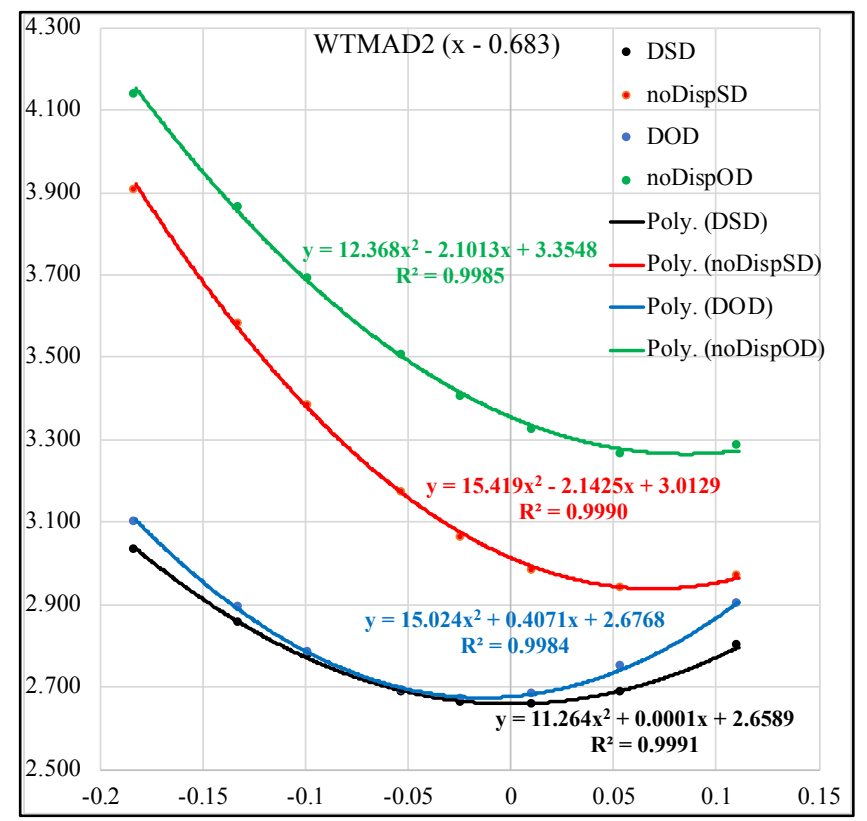

Figure 1: dependence on the fraction of HF exchange $\mathrm{x} / 100 \%$ of WTMAD2 over the GMTKN55 dataset for the dispersion-corrected, spin-component-scaled, double hybrid DSD-SCAN $\mathrm{N}_{\mathrm{x}}-\mathrm{D} 3(\mathrm{BJ})$, as well as the constrained versions DOD (i.e, $\mathrm{c}_{2 \mathrm{ss}}=0$ ), noDispSD (i.e., $\mathrm{s}_{6}=0$ ), and noDispOD (i.e., $\mathrm{s}_{6}=\mathrm{c}_{2 s \mathrm{~s}}=0$ )

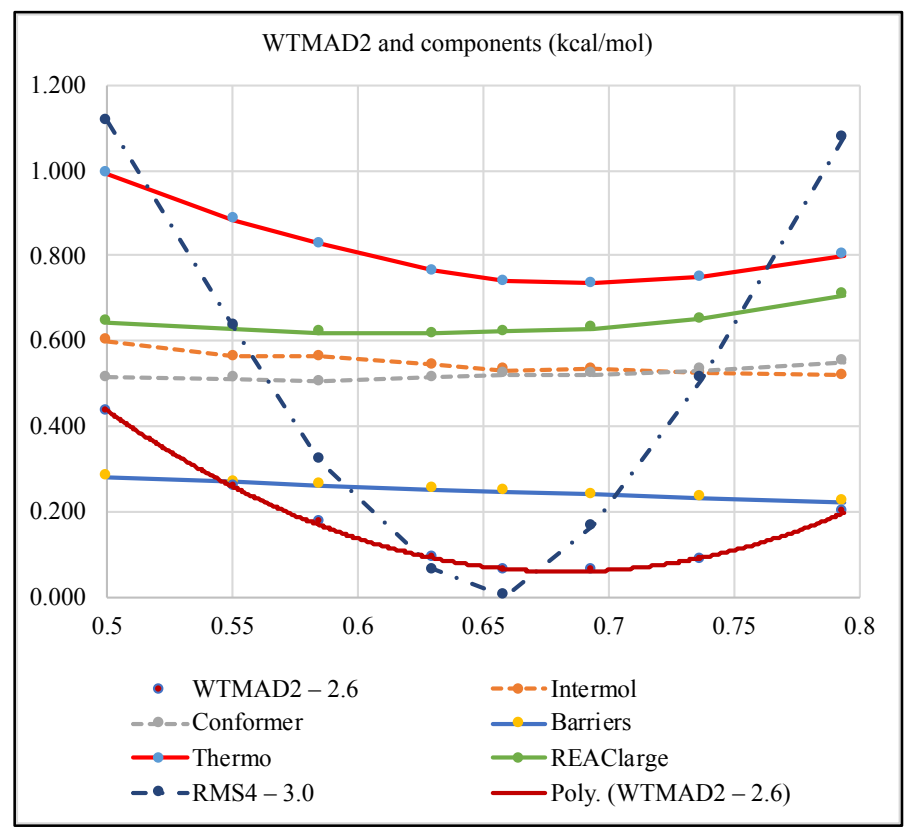

Figure 2: dependence on the fraction of HF exchange $\mathrm{x} / 100 \%$ of WTMAD2 over the GMTKN55 dataset for the dispersion-corrected, spin-component-scaled, double hybrid DSD-SCAN $\mathrm{x}_{\mathrm{x}} \mathrm{D} 3(\mathrm{BJ})$, as well as of the five major subdivisions thereof and of an objective function similar to Ref. ${ }^{37}$ ("RMS4") 
The DOD-SCAN-D3(BJ) curve (blue) - where $\mathrm{c}_{2 \mathrm{ss}}=0$ throughout - is only somewhat less flat, with a minimum at $\mathrm{x}=0.66$. We note that where $\mathrm{x}<0.63$ (the crossing point between DSD and DOD WTMAD2 curves), an unconstrained DSD fit actually has a negative $\mathrm{c}_{2 \mathrm{ss}}$ - if $\mathrm{c}_{2 \mathrm{ss}}$ were constrained to be non-negative, then DSD would follow the blue line left of the crossing point. If the empirical dispersion correction is eliminated (which we denote here by noDispSD-SCAN), the curve does become a little steeper and the minimum shifts up to $\mathrm{x}=0.75$. If we in addition constrain $\mathrm{c}_{2 \mathrm{ss}}=0$ (the yellow noDispOD-SCAN curve), we pay a relatively modest accuracy premium - but this is specific to the underlying SCAN semilocal functional. We will address this point further below.

Of course, a single global performance metric such as WTMAD2 does not tell the whole story. A breakdown by components is given in Tables S2 through S10. A number of these subsets are essentially indifferent to the fraction of HF exchange (particularly the noncovalent interaction sets), while others prefer small HF exchange (e.g. DC13=thirteen "difficult cases"), yet others (e.g., SIE=self-interaction error) will prefer large HF exchange by design, and a number of the thermochemistry subsets have clearly defined minima.

Following the original GMTKN55 paper and the previous section, we can partition WTMAD2 into the same five primary components. A plot of these as a function of $\mathrm{c}_{\mathrm{X}, \mathrm{HF}}$ is given in Figure 2.

We see there that of these five primary subsets, the two noncovalent interaction sets (intramolecular and intermolecular) display some (opposite) variation, but their average NCI/2 is remarkably insensitive to the percentage of HF exchange. The greatest variation is seen for thermochemistry, where a clear "valley" exists, which is however nearly flat between 0.66 and 0.74. For barrier heights, error goes down slowly but monotonically as $\mathrm{c}_{\mathrm{X}, \mathrm{HF}}$ goes up. Finally, for the large reaction energies, the profile stays fairly flat but error starts increasing for the largest values. The combination of these factors creates something of a "Goldilocks zone" between 0.65 and 0.70 , or even between 0.62 and 0.74 , as we noted earlier: for instance, while 0.69 yields slightly better thermochemistry and barrier heights than 0.66 , this is offset by increased errors in the large system isomerizations.

The GMTKN55 minimum is even more shallow than what we previously found ${ }^{37}$ in the DSD paper. We do note that instead of weighted MADs, that paper used unweighted RMSDs, which tends to amplify differences; moreover, it focused on a training set with just six subsets. Two of these were transition metal reaction prototypes; the remaining four are identical or similar to the W4-11, BH76, S22, and MB18 subsets from GMTKN55. RMSD4, an average of the latter four's RMSDs, which should behave quite similarly to the training set used in Ref. ${ }^{37}$, is displayed as the dot-dashed black line in Figure 2. One indeed sees a much more pronounced variation here, as differences are neither smoothed out by the more robust (in the statistical sense) MAD averaging nor diluted by many noncovalent interaction-driven subsets.

We hence conclude that we may avoid the costly nonlinear optimization of $\mathrm{c}_{\mathrm{X}, \mathrm{HF}}$ for the other DSD-XC functionals, and that we may instead semi-arbitrarily fix $\mathrm{c}_{\mathrm{X}, \mathrm{HF}}$ at either the same value as the original, ${ }^{37}$ or choose $0.69 \approx 3^{-1 / 3}$ as a sensible compromise.

The evolution of the parameters in DSD-SCANx as a function of $x=c X, H F$ can be seen in Figure S1 in the Supporting Information. Suffice here to say that, for this particular semilocal functional (SCAN) and the DSD form, dependence of all four parameters $\mathrm{c}_{\mathrm{C}, \mathrm{DFT}}, \mathrm{c}_{2 \mathrm{ab}}, \mathrm{c}_{2 \mathrm{ss}}$, and $\mathrm{s}_{6}$ is remarkably linear over the $0.5 \leq \mathrm{x} \leq 0.8$ range. This empirical behavior is somewhat at odds with the cubic dependence argued on theoretical grounds: ${ }^{101,103}$ we will explore this in more detail in a future paper. 


\section{$\underline{\text { DSD double hybrids and refits }}$}

Performance statistics for DSD functionals with original parametrization were already given in the GMTKN55 paper: differences with the values reported there are principally due to slight differences in the basis set and choices of frozen-cores. In the present paper, we will use the notation, e.g., DSD-PBEP86-D3(BJ) for the original functional and revDSD-PBEP86-D3(BJ) for the present refit. WTMAD2 statistics can be found in Table 1.

With valence electrons correlated, revDSD-PBE86-D3(BJ) and revDSD-BLYP-D3(BJ) are essentially tied at WTMAD2 $=2.42$ and $2.48 \mathrm{kcal} / \mathrm{mol}$, respectively. While this is still higher than $\omega \mathrm{B} 97 \mathrm{M}(2)$, it should be kept in mind that DSD-BLYP-D3refit and DSD-PBEP86-D3refit only entail six adjustable parameters rather than sixteen, rendering them less "empirical". Of these six, $\mathrm{c}_{\mathrm{X}, \mathrm{HF}}$ can be fixed at a semi-arbitrary value; furthermore, we found here that the same is true of the damping function turnover point $\mathrm{a}_{2}$ of the $\mathrm{D} 3(\mathrm{BJ})$ dispersion term. In fact, the surface is so flat in $\mathrm{a}_{2}$ that its inclusion as an optimization parameter leads to poor convergence of WTMAD2: we hence fixed $\mathrm{a}_{2}$ at semi-arbitrary value of 5.5 for most functionals, 5.2 for short-ranged correlation functionals like LYP and P86, and 5.75 for the longer-ranged SCAN (Table S16).

This leaves us arguably with only four true empirical parameters.

It was previously noted, in the original DSD papers, that "upgrading" the underlying semilocal functional from a GGA to a meta-GGA is not necessarily beneficial, with DSD-TPSSD3refit for instance being among the poorer performers. At first sight, this observation holds true here as well: it is also notable that DSD-PBE-D3refit does noticeably more poorly than DSDPBEP86-D3refit and DSD-BLYP-D3refit. What the two best performers have in common are fairly "short-ranged" semilocal correlation functionals, which at long range at least "do no harm" and leave the treatment of dispersion up to the MP2-like correlation and the D3(BJ) correction. In contrast, as noted in Ref. ${ }^{104}$, the $\mathrm{PBE}_{\mathrm{c}}$ correlation functional exhibits a spurious attraction at long range, and so does TPSS.

The very recent SCAN (strongly constrained and appropriately normed ${ }^{10}$ ) meta-GGA, in contrast, exhibits much better performance in a DSD context than TPSS - second only to DSDBLYP and DSD-PBEP86.

The great improvement from the original DSD-PBEP86-D3 (WTMAD2=3.10 kcal $/ \mathrm{mol}$ ) to revDSD-PBEP86-D3 serves as a cautionary tale against small and idiosyncratic training sets. All four of the main-group training sets for the DSD functional are part of GMTKN55: if we were to instead consider the sum of the four WTMAD2 contributions for W4-11, RG18, S22, and BH76, we would actually find essentially no improvement from DSD-PBEP86-D3 to revDSD-PBEP86-D3: the large-system subsets are what makes the difference.

Is there any benefit to be gained from correlating the inner-shell orbitals? (We have noted previously $^{38}$ that DSD-BLYP parameters fitted with and without inner-shell correlation are slightly different.) We have only considered this for a subset of double hybrids. (See also Tables S13 and S14 for sample component breakdowns.) As seen in Table 1, the impact of including inner-shell correlation is quite minor for both DSD and DOD, but for noDisp (see next subsection), where the empirical dispersion correction has been eliminated, including inner-shell correlation systematically improves WTMAD2 by $0.1-0.2 \mathrm{kcal} / \mathrm{mol}$.

The popular simple hybrids B3LYP-D3(BJ) and PBE0-D3(BJ) are nearly tied at WTMAD2 $=6.5 \mathrm{kcal} / \mathrm{mol}$. Replacing PBE exchange by Weitao Yang's revision (revPBE ${ }^{91}$ ) leads 
to a significant improvement to WTMAD2=5.43 $\mathrm{kcal} / \mathrm{mol}$ for revPBE0-D3(BJ). (A detailed comparison can be found in Table S15. This functional was not considered in the GMTKN55 paper.) Intriguingly, making a similar substitution in the double hybrid to create DSDrevPBEP86-D3 does not yield improved performance (WTMAD2=2.66 kcal $/ \mathrm{mol}$, compared to 2.46 for DSD-PBEP86-D3 - both without frozen cores).

At the end of this section, we can report that and revDSD-PBEP86-D3(BJ) is the best performer, closely followed by revDSD-BLYP-D3.

\section{Eliminating the semiempirical dispersion correction}

The presence of the D3(BJ) dispersion correction ${ }^{31,32}$ in DSD functionals exposes them to the criticism of "mixing DFT with molecular mechanics". We have earlier considered the option of eliminating D3(BJ) entirely: in practice, this entails an increased percentage of same-spin correlation as compensation.

As can be seen in Table 1, all the DSD-noD3 (or, if you like, noDispSD) functionals exhibit significantly degraded performance compared to their DSD-D3 siblings. In addition, however, the ordering is upended: noDispSD-SCAN now exhibits significantly better performance than the other options. With core-valence correlation included, noDispSD-SCANQIDH reaches WTMAD2=2.84 kcal $/ \mathrm{mol}$, still significantly better than the original DSDPBEP86-D3.

Why, in a noDispSD-XC functional, does $\mathrm{XC}=\mathrm{SCAN}$ outperform all other options? At long range, three scenarios are possible: (a) the functional tapers away quickly (like in BLYP and PBEP86), which at least does no harm but in the absence of a D3(BJ) correction leaves PT2 to handle all the long-range dispersion effects (for which it is inadequate); (b) the functional does not decay quickly but has the wrong behavior, leading to poor performance for noncovalent interactions; (c) the functional can at least partly recover the correct behavior, in which case PT2 may be sufficient to handle the remainder. It appears that (c) is the case for SCAN.

As a proxy for behavior at intermediate distance, we may consider the $\mathrm{s}_{8}$ coefficient for the $\mathrm{r}^{-8}$ term in D3(BJ): for MP2, this was found ${ }^{69}$ to be large and negative, while for DSD double hybrids and functionals like M06, s8 was found to be zero or statistically insignificantly different from zero. We note in particular that SCAN-D3(BJ), unlike other GGAs and metaGGA, has a fitted $\mathrm{s}_{8}=0$ in the D3(BJ) correction $^{87}$; in the present work, we also fitted a D3(BJ) correction to the SCAN0 hybrid, and obtained $\mathrm{a}_{1}=0, \mathrm{a}_{2}=7.9042, \mathrm{~s}_{8}=0$.

Somewhat at odds with this argument, however, is $\omega \mathrm{B} 97 \mathrm{X}-2$. Upon reoptimizing the prefactor for DFT correlation as well as c2ab and c2ss, we found that WTMAD2=3.06 dropped to $2.80 \mathrm{kcal} / \mathrm{mol}$ whether or not the $\mathrm{D} 3(\mathrm{BJ})$ was included. (The $\mathrm{a}_{1}$ and $\mathrm{a}_{2}$ damping function parameters taken from NG place the correction very far out in space.) This it becomes our best performer in the absence of a dispersion correction.

Another approach to eliminating the D3(BJ) correction would be to replace it with a nonlocal dispersion functional, such as the Vydrov-Van Voorhis VV10 ${ }^{35}$ (also known as NL ${ }^{105}$ ) employed in the B97M-V and other Berkeley functionals. Najibi and Goerigk found ${ }^{100}$ that DSDPBEP86-NL (using a damping parameter $b=14.2$ as suggested in Ref. ${ }^{106}$ ) yields WTMAD2=2.84 $\mathrm{kcal} / \mathrm{mol}$. We obtained VV10 corrections for DSD-PBEP86-NL using $b=12.8$ (as obtained in Ref. $^{71}$ for the revised ${ }^{71}$ S66x8 noncovalent interactions benchmark, ${ }^{107}$ near the basis set limit), then used those to fit a revDSD-PBEP86-NL functional. Its parameters can be found in Table 3: at WTMAD2 $=2.60 \mathrm{kcal} / \mathrm{mol}$, this is within statistical noise from revDSD-PBEP86-D3(BJ), 
illustrating the ability of the DSD refitting process to overcome certain deficiencies in the noncovalent interaction description.

\section{DOD double hybrids and refit}

If only opposite-spin MP2 correlation is included, then the cost scaling of the post-KS step can be reduced to $\propto \mathrm{N}^{4}$ formally by means of a Laplace transform algorithm. ${ }^{108-110} \mathrm{In}$ fact, Song and Martinez ${ }^{111}$ achieved further reduction to $\propto \mathrm{N}^{3}$ using tensor hypercontraction techniques. In our previous work, ${ }^{37}$ we have denoted such functionals DOD, short for Dispersion-corrected, Opposite-spin, Double-hybrids.

(As was expected and can be seen in Table 1, the results of trying to eliminate both samespin correlation and the dispersion correction were dismal for most functionals, with the exception of noDispOD-SCAN 74 where WTMAD=3.3 $\mathrm{kcal} / \mathrm{mol}$. Still, this is a performance comparable with $\omega \mathrm{B} 97 \mathrm{M}-\mathrm{V}$, which does not require evaluation of $\mathrm{E}_{2 \mathrm{ab} .}$ )

The tie between revDSD-BLYP and revDSD-PBEP86 is broken in favor of the latter: Inspection reveals that revDSD-PBEP86-D3 functionals has a $\mathrm{c}_{2 \mathrm{ss}}$ coefficient close to zero, which is not the case for revDSD-BLYP. (The latter is plausible when one considers that BLYP does not treat opposite-spin and same-spin correlation on the same footing: in fact, it is easily seen from eq. (2) in Ref. ${ }^{112}$ that the BLYP correlation energy for a fully polarized uniform electron gas is zero, which is clearly an unphysical answer.) Hence, we see that revDODPBEP86-D3 "pulls ahead of" revDOD-BLYP-D3in the "WTMAD2 race", at 2.45 vs. 2.66 $\mathrm{kcal} / \mathrm{mol}$ (Table 1).

revDOD-PBEP86-NL (Table 3) is essentially indistinguishable in performance from revDOD-PBEP86-D3(BJ).

\section{Final recommended D3(BJ) functionals}

In light of the above, we only are retaining three exchange-correlation combinations for the semilocal part: BLYP, PBEP86, and SCAN. For PBEP86, both DSD and DOD combinations are given: while DSD represents only a very small improvement over DOD, it comes at zero additional computational expense when using a code that cannot exploit reduced-scaling algorithms for opposite-spin-only MP2. (The most commonly used such codes are Gaussian 09 and Gaussian 16.) Hence, we have elected to recommend both revDSD-PBEP86-D3 and revDOD-PBEP86-D3.

In view of the weak dependence of performance on the percentage of HF exchange, we have elected to retain the original percentages of $71 \%$ for DSD-BLYP and $69 \%$ for DSDPBEP86, in order to simplify nonstandard inputs for codes such as Gaussian, ${ }^{113}$ ORCA, ${ }^{114,115}$ and Q-CHEM. For DSD-SCAN and DOD-SCAN, we have no such incentive: following inspection of the minima of the parabolic fits to WTMAD2, we have chosen $66 \%$ for DOD-SCAN and $69 \%$ for noDispSD-SCAN.

Finally, we have noted above that, as long as the dispersion prefactor $\mathrm{s}_{6}$ is selfconsistently optimized with the other parameters, WTMAD2 is only weakly dependent on the chosen turnover parameter $\mathrm{a}_{2}$. With a given fixed $\mathrm{a}_{2}$, DSD-BLYP has the largest $\mathrm{s}_{6}$, followed by DSD-PBEP86: for DSD-SCAN and DOD-SCAN, $\mathrm{s}_{6}$ is much smaller - this reflects that SCAN is better able to handle long-range effects than the two others. We have hence semi-arbitrarily fixed 
$\mathrm{a}_{2}$ at a "short" value of 5.2 for DSD-BLYP, and at a "longer" values of 5.75 for DSD-SCAN, while for DSD-PBEP86 we chose an intermediate $\mathrm{a}_{2}=5.5$. Comparison with full optimizations including a revealed that WTMAD2 differences are on the other of a few "small" calories per mole: hence fixing these parameters is considered justifiable in light of a more smoothly converging optimization for the remaining ones.

Table 3: Final parameters for revised DSD-D3(BJ) functionals and revDSD-PBEP86-NL. Original parameters, if any, given for comparison

\begin{tabular}{|c|c|c|c|c|c|c|c|c|}
\hline \multirow[t]{2}{*}{ Functionals } & \multirow[t]{2}{*}{ CX,HF } & \multirow[t]{2}{*}{ CC,DFT } & \multirow[t]{2}{*}{$\mathbf{c}_{2 a b}$} & \multirow[t]{2}{*}{ c2ss } & \multirow[t]{2}{*}{ S6 } & \multirow[t]{2}{*}{$\mathbf{a}_{1}$} & \multirow[t]{2}{*}{ a2 } & \multirow{2}{*}{$\begin{array}{l}\text { WTMAD2 } \\
\text { (kcal/mol) }\end{array}$} \\
\hline & & & & & & & & \\
\hline noDispSD-SCAN $_{69}$ & 0.69 & 0.4409 & 0.6223 & 0.26 & [0] & - & - & 2.98 \\
\hline DOD-SCAN $_{66}-\mathrm{D} 3(\mathrm{BJ})$ & 0.66 & 0.5048 & 0.6283 & {$[0]$} & 0.3152 & {$[0]$} & 5.75 & 2.67 \\
\hline revDSD-BLYP-D3(BJ) & 0.71 & 0.5313 & 0.5477 & 0.1979 & 0.5451 & {$[0]$} & 5.2 & 2.48 \\
\hline revDSD-PBEP86-D3(BJ) & 0.69 & 0.4296 & 0.5785 & 0.0799 & 0.4377 & {$[0]$} & 5.5 & 2.42 \\
\hline revDSD-PBEP86-NL & 0.69 & 0.435 & 0.5762 & 0.0622 & 0.9921 & $\mathrm{~b}=14.2$ & - & 2.44 \\
\hline revDSD-PBEB95-D3(BJ) & 0.66 & 0.4960 & 0.4935 & 0.1009 & 0.3686 & {$[0]$} & 5.5 & 2.85 \\
\hline revDSD-PBE-D3(BJ) & 0.68 & 0.4528 & 0.5845 & 0.0711 & 0.5746 & [0] & 5.5 & 2.72 \\
\hline revDOD-BLYP-D3(BJ) & 0.71 & 0.5911 & 0.6216 & {$[0]$} & 0.6145 & {$[0]$} & 5.2 & 2.67 \\
\hline revDOD-PBEP86-D3(BJ) & 0.69 & 0.4449 & 0.6055 & {$[0]$} & 0.477 & [0] & 5.5 & 2.47 \\
\hline revDOD-PBEP86-NL & 0.69 & 0.4445 & 0.5994 & {$[0]$} & 1.0629 & $\mathrm{~b}=14.2$ & - & 2.46 \\
\hline revDOD-PBEB95-D3(BJ) & 0.66 & 0.5225 & 0.5278 & {$[0]$} & 0.4107 & {$[0]$} & 5.5 & 2.92 \\
\hline revDOD-PBE-D3(BJ) & 0.68 & 0.4641 & 0.6134 & {$[0]$} & 0.6067 & {$[0]$} & 5.5 & 2.73 \\
\hline 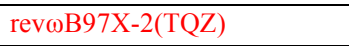 & (b) & 0.9518 & 0.5123 & 0.4294 & {$[0]$} & - & - & 2.80 \\
\hline Orig. ${ }^{37}$ DSD-BLYP-D3(BJ) & 0.71 & 0.54 & 0.47 & 0.4 & 0.57 & {$[0]$} & 5.4 & 3.34 \\
\hline Orig. ${ }^{37}$ DSD-PBEP86-D3(BJ) & 0.69 & 0.44 & 0.52 & 0.22 & 0.48 & {$[0]$} & 5.6 & 3.10 \\
\hline Orig. ${ }^{37}$ DSD-PBEB95-D3(BJ) & 0.66 & 0.55 & 0.46 & 0.09 & 0.61 & {$[0]$} & 6.2 & 3.32 \\
\hline Orig. ${ }^{37}$ DSD-PBE-D3(BJ) & 0.68 & 0.49 & 0.55 & 0.13 & 0.78 & {$[0]$} & 6.1 & 3.17 \\
\hline DSD-PBEP86-NL ${ }^{(a)}$ & 0.69 & 0.44 & 0.52 & 0.22 & {$[1.0]$} & $\mathrm{b}=14.2$ & - & 2.60 \\
\hline B2GP-PLYP-D3(BJ) ${ }^{39,55}$ & 0.65 & 0.64 & 0.36 & 0.36 & 0.56 & 0.2597 & 6.333 & 3.19 \\
\hline
\end{tabular}

(a) calculated using ORCA $4.1,{ }^{115}$ this work

(b) short-range 0.6362 , long-range 1.0 , range separation parameter $\omega=0.3$.

Sample input files for most major codes are given in the ESI. We wish to point out that, while noDispSD-SCAN is inferior to the revDSD offerings, its WTMAD2 is still superior to the original B2GP-PLYP and DSD double hybrids, and this without any empirical dispersion correction and with just three non-arbitrary parameters.

\section{Considering the D4 dispersion correction: Final recommended D4 functionals}

As the present manuscript was being prepared for publication, a preprint ${ }^{34}$ by Grimme and coworkers was posted on ChemRXiv, in which they propose a next-generation D4 dispersion correction (see also Ref. ${ }^{33}$ ). The reader is referred to these references for details; for the purpose of our discussion, the most significant difference between D3(BJ) and D4 is that the latter introduces dependence on atomic partial charges, which (by default) are evaluated using the electronegativity equalization principle. ${ }^{116}$ (For the general theory, see Refs. ${ }^{117,118}$ and references therein.) 
As a first step, we substituted the D4 correction for D3(BJ) in the original DSD functionals from Ref. ${ }^{37}$ as a "drop-in replacement" using parameters optimized for these functionals and published by Grimme et al. ${ }^{34}$ The results can be found in the third numerical column of Table 4 and, for individual GMTKN55 subsets, in Table S13. Across the board, the WTMAD2 values are significantly better than those with the original, in the case of DSD-PBE even superior to the refitted revDSD-PBE-D3(BJ)!

We then proceeded to reoptimize the DSD functionals in the presence of D4 and adjusting the latter's parameters. It quickly became clear that setting s8 to zero had negligible impact on the WTMAD2: furthermore, that the other parameters settled around $\mathrm{a}_{1}=0.4$ and $\mathrm{a}_{2}=3.6$, and that one could actually choose these 'semi-arbitrary values' across the board, leaving the same four adjustable parameters $\mathrm{c}_{\mathrm{C}, \mathrm{DFT}}, \mathrm{c}_{2 \mathrm{ab}}, \mathrm{c}_{2 \mathrm{ss}}$, and $\mathrm{s}_{6}$ as in the revDSD-XC-D3(BJ) cases.

revDSD-PBEP86-D4 in particular shines, with WTMAD2 $=2.33 \mathrm{kcal} / \mathrm{mol}$, quite close to the $\omega \mathrm{B} 97 \mathrm{M}(2)$ functional with its 16 adjustable parameters. But revDSD-SCAN $66-\mathrm{D} 4$, revDSDPBE-D4, and revDSD-PBEB95-D4 all likewise outperform their revDSD-XC-D3(BJ) counterparts, and revDSD-BLYP-D4 marginally bests revDSD-BLYP-D3(BJ).

Three of the refitted functionals have $\mathrm{c}_{2 \mathrm{ss}}$ values close to zero, hence we also performed revDOD-PBE-D4, DOD-SCAN66-D4, and revDOD-PBEB95-D4 fits in case one wants to exploit the reduced-scaling algorithms for opposite-spin-only MP2. For revDOD-BLYP-D4, there is substantial loss in performance, but revDOD-PBEP86-D4, at WTMAD2=2.36 kcal $/ \mathrm{mol}$, only sacrifices $0.03 \mathrm{kcal} / \mathrm{mol}$ compared to its DSD counterpart. Next in performance is revDOD-PBED4 at WTMAD2 $=2.47 \mathrm{kcal} / \mathrm{mol}$, then followed by DOD-SCAN-D4 at $2.64 \mathrm{kcal} / \mathrm{mol}$.

Inspection of the contributions to WTMAD2 (Table S17) reveals that the improvement from revDSD-PBEP86-D3(BJ) to revDSD-PBEP86-D4 is mostly due to the intermolecular interaction components, and somewhat due to conformers. In DOD-SCAN66-D4, on the other hand, intermolecular interactions and conformers are improved about equally.

While it is easy to rationalize the improvement in WTMAD2 from revDSD-D3(BJ) to revDSD-D4, it is not so obvious why the WTMAD2 of revDSD-PBEP86-D4 would be lower still than that of revDSD-PBEP86-NL. Detailed comparison reveals (Table S13) that four of the 55 subsets account for nearly all the difference: HAL59 (halogen bonding), HEAVY28 (NCI energies involving heavy p-block elements other than halogens), AMINO20X4 (Relative energies in amino acid conformers) and PNICO23(Interaction energies in pnicogen-containing dimers). All of these have small average reaction energies and thus large weights in the WTMAD2 formula, Eq. (2), which means that small changes in the description of these four subsets have a disproportionately large impact on WTMAD2. (We note that DSD-PBEP86-D4 likewise has a small edge over DSD-PBEP86-NL.) For the remaining 51 subsets, an accuracy

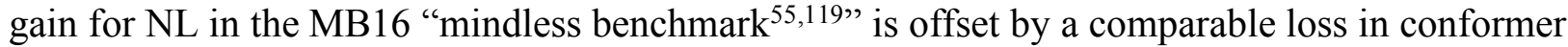
energies for melatonin, leaving the two WTMAD2 totals within $0.10 \mathrm{kcal} / \mathrm{mol}$ of each other. While it is clear that both D4 and NL represent substantial improvements over D3(BJ), we have insufficient data to decide whether D4 is superior over NL, or conversely.

Iron and Janes ${ }^{120}$ have very recently examined the performance of hybrid and doublehybrid functionals for their newly developed MOBH35 transition metal barrier heights benchmark as well as for the older MOR41 organometallic reaction energy benchmark. ${ }^{121}$ There, the SCAN-based functionals were found to be superior to the others for these applications, even though overall the $\omega \mathrm{B} 97 \mathrm{M}-\mathrm{V}$ functional outperformed all double hybrids except PWPB95. ${ }^{122}$ Detailed inspection of the double-hybrid results revealed a number of outliers for system that exhibit some degree of static correlation: apparently, the PT2 correlation is insufficiently 
resilient to that. The use of dRPA (direct random phase approximation) as an alternative, as proposed by Mezei et al., ${ }^{123,124}$ will be explored in future work. (The use of perturbation theory higher than second order was considered by Chan and Radom ${ }^{125}$ and found to yield essentially no performance benefit.) Results for the $\omega \mathrm{B} 97 \mathrm{M}(2)$ functional were not given in that paper. For the sake of completeness, we carried out these calculations ourselves using the def2-TZVPP basis set used in Ref. ${ }^{120}$ for the other double hybrids: for MOR41, ${ }^{121}$ we obtain MAD=2.8 and $\mathrm{RMSD}=3.5 \mathrm{kcal} / \mathrm{mol}$, while for $\mathrm{MOBH} 35,{ }^{120}$ we obtain $\mathrm{MAD}=2.4$ and $\mathrm{RMSD}=4.0 \mathrm{kcal} / \mathrm{mol}$.

Table 4: Final parameters for revDSD-D4 functionals and comparison of WTMAD2 (kcal/mol) with original double-hybrids (D3(BJ)), ditto with drop-in replacement of D3(BJ) by D4, and revD3(BJ)

\begin{tabular}{|c|c|c|c|c|c|c|c|c|c|c|c|c|}
\hline \multirow[t]{2}{*}{ Functionals } & \multicolumn{4}{|c|}{ WTMAD2(kcal/mol) } & \multicolumn{8}{|c|}{ Parameters } \\
\hline & D3(BJ) & revD3(BJ) & D4 & revD4 & cX,HF & eC,DFT & $\mathbf{c}_{2 a b}$ & $\mathbf{c}_{2 s s}$ & S6 & s8 & $\mathbf{a}_{1}$ & $\mathbf{a}_{2}$ \\
\hline DSD-PBEP86 & 3.099 & 2.422 & 2.649 & 2.332 & 0.69 & 0.4210 & 0.5922 & 0.0636 & 0.5132 & 0 & 0.44 & 3.60 \\
\hline $\begin{array}{l}\text { With core corr } \\
\text { DSD-PBEP86 }\end{array}$ & & & & 2.307 & 0.69 & 0.4038 & 0.5979 & 0.0571 & 0.4612 & 0 & 0.44 & 3.60 \\
\hline DSD-PBE & 3.170 & 2.738 & 2.637 & 2.461 & 0.68 & 0.4403 & 0.6025 & 0.0417 & 0.6706 & 0 & 0.4 & 3.6 \\
\hline DSD-BLYP & 3.336 & 2.484 & 2.829 & 2.592 & 0.71 & 0.5169 & 0.5586 & 0.1972 & 0.6141 & 0 & 0.38 & 3.52 \\
\hline DSD-SCAN & - & 2.662 & - & 2.635 & 0.66 & 0.4855 & 0.6320 & 0.0131 & 0.3203 & 0 & 0.4 & 3.6 \\
\hline DSD-PBEB95 & 3.325 & 2.845 & 3.109 & 2.700 & 0.66 & 0.4549 & 0.5305 & 0.0547 & 0.4707 & 0 & 0.42 & 2.93 \\
\hline xDSD-PBEP86 & & & & 2.318 & 0.69 & $0.4155^{\mathrm{a}}$ & 0.6023 & 0.0514 & 0.4829 & 0 & 0.44 & 3.60 \\
\hline Ditto, $s 8 \neq 0$ & & & & 2.302 & 0.69 & 0.4135 & 0.6044 & 0.0476 & 0.4158 & 0.1096 & 0.44 & 3.60 \\
\hline $\begin{array}{l}\text { With core corr } \\
\text { xDSD-PBEP86 }\end{array}$ & & & & 2.229 & 0.69 & $0.3986^{\mathrm{a}}$ & 0.6077 & 0.0502 & 0.4200 & 0 & 0.44 & 3.60 \\
\hline $\begin{array}{l}\text { With core corr } \\
\text { xDOD-PBEP86 }\end{array}$ & & & & 2.247 & 0.69 & $0.4071^{\mathrm{a}}$ & 0.6261 & 0 & 0.4561 & 0 & 0.44 & 3.60 \\
\hline DOD-PBEP86 & & & & 2.363 & 0.69 & 0.4323 & 0.6122 & 0 & 0.5552 & 0 & 0.44 & 3.60 \\
\hline DOD-PBE & & & & 2.470 & 0.68 & 0.4470 & 0.6181 & 0 & 0.6992 & 0 & 0.4 & 3.6 \\
\hline DOD-SCAN & & & & 2.637 & 0.66 & 0.4914 & 0.6344 & 0 & 0.3270 & 0 & 0.4 & 3.6 \\
\hline DOD-PBEB95 & & & & 2.714 & 0.66 & 0.4653 & 0.5532 & 0 & 0.4915 & 0 & 0.42 & 2.93 \\
\hline DOD-BLYP & & & & 2.792 & 0.71 & 0.5619 & 0.6346 & 0 & 0.7105 & 0 & 0.38 & 3.52 \\
\hline
\end{tabular}

a. During iterations, $\mathrm{C}_{\mathrm{C}, \mathrm{DFT}}=1.00$ as for all $\mathrm{xDSD}$ functionals

Coming back to GMTKN55: can we improve further over revDSD-PBEP86-D4, at WTMAD2=2.33 kcal/mol? As above, we found a minor improvement over revDSD-PBEP86$\mathrm{D} 3(\mathrm{BJ})$ when the frozen-core approximation was not made, we attempted the same here, and found that revDSD-PBEP86-D4(noFC) [where noFC stands for "no frozen cores"] has a slightly lower WTMAD2=2.31 kcal/mol. (See Table S17 for details.)

Then we attempted one more thing that also is present in $\omega \mathrm{B} 97 \mathrm{M}(2)$ : we evaluated the KS orbitals with full DFT correlation, akin to the XYG3 family of functionals ${ }^{40}$. Previously, we have found ${ }^{42}$ for much smaller training sets that (a) typically error metrics go through a minimum when the percentage of HF exchange in the final energy evaluation is at or near that used for determining the orbitals (leading to what we have termed ${ }^{42}$ xDSD functionals); (b) the improvement seen from DSD to xDSD was small and its statistical significance uncertain. Hait and Head-Gordon have discussed some downsides of the XDSD and XYG3 type functionals for nonequilibrium geometries. ${ }^{43}$ It was argued in Ref. ${ }^{122}$ that the putative success of that approach results not from the effect of the un-throttled DFT correlation on the orbitals but from the smaller orbital gaps appearing in the denominators of the MP2-like terms.

In the present work, we have obtained an xrevDSD-PBEP86-D4(noFC) functional fitted to the GMTKN55 dataset. Parameters are given in Table 4: the WTMAD2 obtained, 2.23 $\mathrm{kcal} / \mathrm{mol}$, is the lowest of any functional optimized here, and is statistically equivalent to the 2.18 $\mathrm{kcal} / \mathrm{mol}$ of the highly empirical $\omega B 97 M(2)$ functional despite the much smaller number of 
parameters. Detailed inspection of Table S17 reveals that the improvement over the revDSDPBEP86-D4 functional mostly comes from just one subset, namely, the radical stabilization energies in RSE43. Turning to the five major categories, $\omega \mathrm{B} 97 \mathrm{M}(2)$ outperforms xrevDSDPBEP86-D4(noFC) for thermochemistry and is in turn outperformed for conformer energies, while there is little to choose between them for intermolecular interaction energies, barrier heights, and large system reactions.

Can we eliminate the same-spin correlation and thus enable the reduced-scaling MP2 algorithms (as well as eliminate one more empirical parameter)? As seen in Table 4, the WTMAD2 for xrevDOD-PBEP86-D4(noFC) is just $0.02 \mathrm{kcal} / \mathrm{mol}$ higher at $2.25 \mathrm{kcal} / \mathrm{mol}$, We have, hence, a functional comparable to $\omega \mathrm{B} 97 \mathrm{M}(2)$ in quality that is amenable to reduced $\mathrm{O}\left(\mathrm{N}^{4}\right)$ or $\mathrm{O}\left(\mathrm{N}^{3}\right) \mathrm{MP} 2$ scaling, unlike $\omega \mathrm{B} 97 \mathrm{M}(2)$ which has $\mathrm{c}_{2 \mathrm{ab}}=\mathrm{c}_{2 \mathrm{ss}}$. This may be relevant in application to larger systems than considered presently. (The largest species in GMTKN55 has 'only' 83 atoms.) However, the difference in WTMAD2 between 2.23 for xrev-DSD-PBE86-D4 and 2.33 for rev-DSD-PBEP86-D4 can be argued to be comparable to the uncertainty in the GMTKN55 reference data, and hence should perhaps not be given excess weight.

\section{Harmonic frequencies as a sanity check}

We have previously shown ${ }^{126}$ that for the HFREQ harmonic frequencies benchmark, ${ }^{126}$ the DSD-PBEP86 functional performed exceptionally well (RMSD $=9.8 \mathrm{~cm}-1$ with the defQZVP basis set and a scale factor of 0.9971), unlike earlier double hybrids. As HFREQ samples a different type of information than GMTKN55, it can act as a sanity check on revDSD-PBEP86: if RMSD[HFREQ] were substantially higher than for the original DSD-PBEP86, then perhaps the improved performance for GMTKN55 would reflect improved physics not as much as the famous quip attributed to John von Neumann that "with four parameters, I can fit an elephant, and with five, I can make him wiggle his trunk".

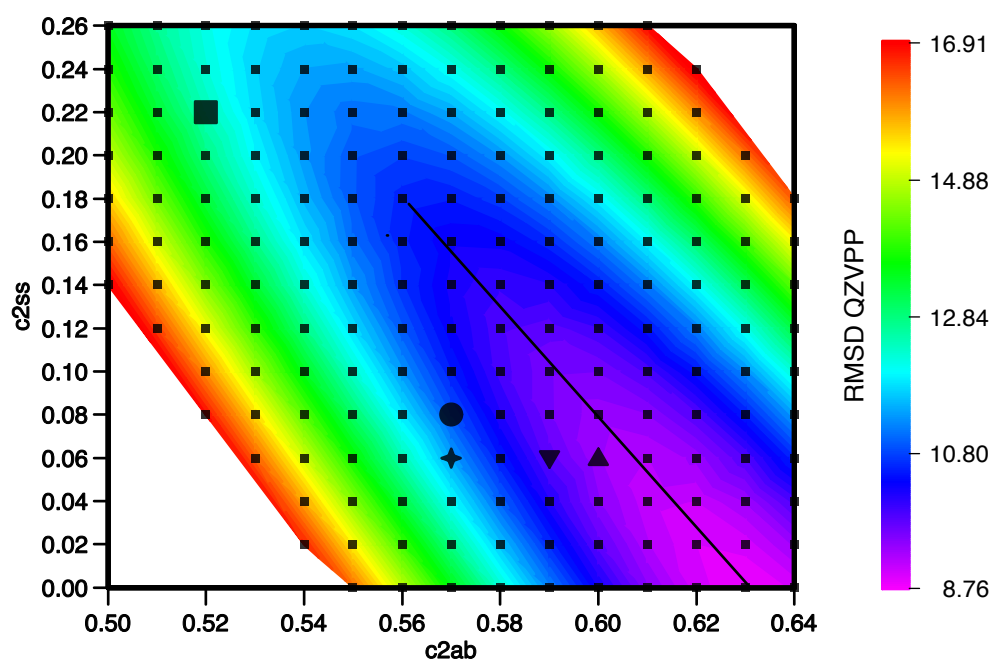

Figure 3: contour plot of RMSD (cm-1) for the HFREQ database for DSD-PBEP86 like forms, as a function of the opposite-spin and same-spin MP2 coefficients $\mathrm{c} 2 \mathrm{ab}$ and $\mathrm{c} 2 \mathrm{ss}$, respectively. The square marker indicates the original DSD-PBEP86-D3(BJ) solution, the large round marker revDSD-PBEP86-D3(BJ), and the triangular ones revDSDPBEP86-D4 on the left and revDOD-PBEP86-D4 on the right. The slanted line only serves to guide the eye. 
As one can see in Figure 3, revDSD-PBEP86-D3(BJ) and especially revDSD-PBEP86D4 in fact show somewhat improved performance for harmonic frequencies. If we fit scaling factors for harmonic frequencies in the same manner as in Ref. ${ }^{126}$, we obtain values that are only semantically different from 1.0.

An additional sanity check is afforded by the work of Iron and Janes ${ }^{120}$ on transition metal barrier heights, and of Efremenko and one of us ${ }^{127}$ on a specific organometallic catalysis problem. ${ }^{128}$ In both cases, the revDSD-PBEP86-D3(BJ) and revDSD-PBEP86-D4 functionals came considerably closer to the coupled cluster benchmark data than the original DSD-PBEP86D3(BJ) parametrization, even though (as noted above) they do not offer the clear added value over $\omega \mathrm{B} 97 \mathrm{M}-\mathrm{V}$ for transition metal systems that they do for main-group systems. Still, our confidence in the reparametrization against GMTKN55 is bolstered by its yielding better performance than the original both when sampling a different type of property (energy derivatives) and when sampling energetic properties for a different sector of chemical space.

A reviewer inquired about best performers for individual subsets. A sorted, color-shaded table with performance metrics for the five major subsets of WTMAD2, as well as for the total, has been given in the ESI as Table S18, with the top three performers for each subset marked. Said showings are largely a back-and-forth between $\omega B$ 97M(2) and revDSD-PBEP86-D4. We should point out that revDSD-PBEP86-D3(BJ) entails a small enough loss in performance (much smaller than between $\omega \mathrm{B} 97 \mathrm{M}-\mathrm{V}$ and $\omega \mathrm{B} 97 \mathrm{M}-\mathrm{D} 3(\mathrm{BJ})$, for instance) that it remains an attractive option, as it can be carried out with existing codes (including all of Gaussian 16, ORCA, and QCHEM) without source code modification and permits analytical first and second derivatives.

\section{Conclusions}

Having made an extensive survey of DSD double hybrid (and some other) functionals with the aid of the GMTKN55 dataset, we are in a position to state the following observations:

- the combinatorially optimized $\omega \mathrm{B} 97 \mathrm{M}-\mathrm{V}$ is "best in class" for fourth-rung exchangecorrelation functionals, and approaches performance of double hybrids like B2GP-PLYP$\mathrm{D} 3(\mathrm{BJ})$;

- the combinatorially optimized $\omega \mathrm{B} 97 \mathrm{M}(2)$ yields the lowest WTMAD2 metric of any functional in existence, making it best in class for double hybrids;

- in a DSD double hybrid context, the very recent D4 dispersion correction is clearly superior over D3(BJ), presumably owing to the newly introduced partial charge dependence;

- the available data suggest that revDSD-PBEP86-D4 is slightly superior to revDSD-PBEP86-

NL;

- while $\omega \mathrm{B} 97 \mathrm{M}(2)$ has sixteen empirical parameters, refitted revDSD-PBEP86-D3(BJ) comes close in performance, while xrevDSD-PBEP86-D4 and xrevDOD-PBEP86-D4 are essentially equivalent in quality to $\omega \mathrm{B} 97 \mathrm{M}(2)$. Out of their reduced number of parameters, the percentage of HF exchange $c_{X, H F}$ and the damping function parameters $a_{1}, a_{2}$ can be fixed at semi-arbitrary values (as the WTMAD2 surface is fairly flat in them), leaving just four true optimization parameters for revDSD and three for revDOD. The revDOD option permits the use of reducedscaling MP2 algorithms, which might prove useful for large systems;

- for the underlying semilocal functional in double hybrids, any good exchange functional appears to work well, while simple correlation functionals that rapidly "get out of the way" at long distances appear to work best (e.g., P86 ${ }^{129}$ and LYP ${ }^{130}$ ); 
- if one wishes to avoid the D3 or D4 corrections, however, DSD-SCAN overall appears to work best. Here, the number of empirical parameters is down to four, one of which (the fraction of HFlike exchange) semi-arbitrary. The best performance without dispersion corrections; - refitting of the DSD functionals to the GMTKN55 database very substantially improves their accuracy particularly for noncovalent interactions and large-system reactions. This serves as a cautionary tale about the use of small, idiosyncratic training sets for empirical functionals.

\section{Supporting Information}

The Supporting Information is available free of charge on the ACS Publications website at DOI: 10.1021/acs.jpca.9b03157.

Tables S1 through S18 (PDF); Figure S1; sample revDSD-PBEP86-D3BJ inputs for several electronic structure codes.

\section{Acknowledgments}

This research was supported by the Israel Science Foundation (grant 1358/15) and by the Minerva Foundation, Munich, Germany, as well as by two internal Weizmann Institute funding sources: the Helen and Martin Kimmel Center for Molecular Design and a research grant from the estate of Emile Mimran. N.S. acknowledges a doctoral fellowship from the Feinberg Graduate School (WIS). The authors would like to thank a referee for helpful comments.

\section{Author Information}

\section{Corresponding Author}

J. M. L. Martin. E-mail: gershom@weizmann.ac.il. Fax: +972 89343029.

\section{ORCID}

Jan M. L. Martin: 0000-0002-0005-5074

Notes

The authors declare no competing financial interest.

\section{References}

(1) Dral, P. O.; Wu, X.; Spörkel, L.; Koslowski, A.; Thiel, W. Semiempirical QuantumChemical Orthogonalization-Corrected Methods: Benchmarks for Ground-State Properties. J. Chem. Theory Comput. 2016, 12 (3), 1097-1120. https://doi.org/10.1021/acs.jctc.5b01047.

(2) DeYonker, N. J.; Cundari, T. R.; Wilson, A. K. The Correlation Consistent Composite Approach (CcCA): Efficient and Pan-Periodic Kinetics and Thermodynamics. In Advances in the Theory of Atomic and Molecular Systems (Progress in Theoretical 
Chemistry and Physics, Vol. 19); Piecuch, P., Maruani, J., Delgado-Barrio, G., Wilson, S., Eds.; Progress in Theoretical Chemistry and Physics; Springer Netherlands: Dordrecht, 2009; Vol. 19, pp 197-224. https://doi.org/10.1007/978-90-481-2596-8_9.

(3) Curtiss, L. A.; Redfern, P. C.; Raghavachari, K. Gaussian-4 Theory. J. Chem. Phys. 2007, 126 (8), 084108. https://doi.org/10.1063/1.2436888.

(4) Montgomery, J. A.; Frisch, M. J.; Ochterski, J. W.; Petersson, G. A. A Complete Basis Set Model Chemistry. VI. Use of Density Functional Geometries and Frequencies. J. Chem. Phys. 1999, 110 (6), 2822. https://doi.org/10.1063/1.477924.

(5) Martin, J. M. L.; de Oliveira, G. Towards Standard Methods for Benchmark Quality Ab Initio Thermochemistry-W1 and W2 Theory. J. Chem. Phys. 1999, 111 (5), 1843-1856. https://doi.org/10.1063/1.479454.

(6) Karton, A.; Martin, J. M. L. Explicitly Correlated W n Theory: W1-F12 and W2-F12. J. Chem. Phys. 2012, 136 (12), 124114. https://doi.org/10.1063/1.3697678.

(7) Ramakrishnan, R.; Dral, P. O.; Rupp, M.; von Lilienfeld, O. A. Big Data Meets Quantum Chemistry Approximations: The $\Delta$-Machine Learning Approach. J. Chem. Theory

Comput. 2015, 11 (5), 2087-2096. https://doi.org/10.1021/acs.jctc.5b00099.

(8) Perdew, J. P.; Burke, K.; Ernzerhof, M. Generalized Gradient Approximation Made Simple. Phys. Rev. Lett. 1996, 77 (18), 3865-3868.

https://doi.org/10.1103/PhysRevLett.77.3865.

(9) Tao, J.; Perdew, J. P.; Staroverov, V. N.; Scuseria, G. E. Climbing the Density Functional Ladder: Nonempirical Meta-Generalized Gradient Approximation Designed for Molecules and Solids. Phys. Rev. Lett. 2003, 91 (14), 146401. https://doi.org/10.1103/PhysRevLett.91.146401.

(10) Sun, J.; Ruzsinszky, A.; Perdew, J. P. Strongly Constrained and Appropriately Normed Semilocal Density Functional. Phys. Rev. Lett. 2015, 115 (3), 036402. https://doi.org/10.1103/PhysRevLett.115.036402.

(11) DeYonker, N. J.; Cundari, T. R.; Wilson, A. K. The Correlation Consistent Composite Approach (CcCA): An Alternative to the Gaussian-n Methods. J. Chem. Phys. 2006, 124 (11), 114104. https://doi.org/10.1063/1.2173988.

(12) Martin, J. M. L.; Parthiban, S. W1 and W2 Theories, and Their Variants:

Thermochemistry in the KJ/Mol Accuracy Range. In Quantum-Mechanical Prediction of Thermochemical Data; Cioslowski, J., Ed.; Understanding Chemical Reactivity; Kluwer Academic Publishers: Dordrecht, 2002; Vol. 22, pp 31-65. https://doi.org/10.1007/0-30647632-0_2.

(13) Becke, A. D. Density-Functional Thermochemistry. V. Systematic Optimization of Exchange-Correlation Functionals. J. Chem. Phys. 1997, 107 (20), 8554-8560. https://doi.org/10.1063/1.475007.

(14) Hamprecht, F. A.; Cohen, A. J.; Tozer, D. J.; Handy, N. C. Development and Assessment of New Exchange-Correlation Functionals. J. Chem. Phys. 1998, 109 (15), 6264-6271. https://doi.org/10.1063/1.477267.

(15) Boese, A. D.; Martin, J. M. L. Development of Density Functionals for Thermochemical Kinetics. J. Chem. Phys. 2004, 121 (8), 3405-3416. https://doi.org/10.1063/1.1774975.

(16) Zhao, Y.; Truhlar, D. G. Density Functionals with Broad Applicability in Chemistry. Acc. Chem. Res. 2008, 41 (2), 157-167. https://doi.org/10.1021/ar700111a.

(17) Yu, H. S.; He, X.; Li, S. L.; Truhlar, D. G. MN15: A Kohn-Sham Global-Hybrid Exchange-Correlation Density Functional with Broad Accuracy for Multi-Reference and 
Single-Reference Systems and Noncovalent Interactions. Chem. Sci. 2016, 7 (8), 5032 5051. https://doi.org/10.1039/C6SC00705H.

(18) Becke, A. D. Density-functional Thermochemistry. III. The Role of Exact Exchange. $J$. Chem. Phys. 1993, 98 (7), 5648-5652. https://doi.org/10.1063/1.464913.

(19) Adamson, R. D.; Gill, P. M. W.; Pople, J. A. Empirical Density Functionals. Chem. Phys. Lett. 1998, 284 (1-2), 6-11. https://doi.org/10.1016/S0009-2614(97)01282-7.

(20) Boese, A. D.; Handy, N. C. A New Parametrization of Exchange-Correlation Generalized Gradient Approximation Functionals. J. Chem. Phys. 2001, 114 (13), 5497-5503. https://doi.org/10.1063/1.1347371.

(21) Mardirossian, N.; Head-Gordon, M. SB97X-V: A 10-Parameter, Range-Separated Hybrid, Generalized Gradient Approximation Density Functional with Nonlocal Correlation, Designed by a Survival-of-the-Fittest Strategy. Phys. Chem. Chem. Phys. 2014, 16 (21), 9904-9924. https://doi.org/10.1039/c3cp54374a.

(22) Mardirossian, N.; Head-Gordon, M. Mapping the Genome of Meta-Generalized Gradient Approximation Density Functionals: The Search for B97M-V. J. Chem. Phys. 2015, 142 (7), 074111. https://doi.org/10.1063/1.4907719.

(23) Mardirossian, N.; Head-Gordon, M. $\Omega$ B97M-V: A Combinatorially Optimized, RangeSeparated Hybrid, Meta-GGA Density Functional with VV10 Nonlocal Correlation. $J$. Chem. Phys. 2016, 144 (21), 214110. https://doi.org/10.1063/1.4952647.

(24) Mardirossian, N.; Head-Gordon, M. Survival of the Most Transferable at the Top of Jacob's Ladder: Defining and Testing the $\Omega$ B97M(2) Double Hybrid Density Functional. J. Chem. Phys. 2018, 148 (24), 241736. https://doi.org/10.1063/1.5025226.

(25) Perdew, J. P.; Schmidt, K. Jacob's Ladder of Density Functional Approximations for the Exchange-Correlation Energy. AIP Conf. Proc. 2001, 577 (1), 1-20. https://doi.org/10.1063/1.1390175.

(26) Kümmel, S.; Kronik, L. Orbital-Dependent Density Functionals: Theory and Applications. Rev. Mod. Phys. 2008, 80 (1), 3-60. https://doi.org/10.1103/RevModPhys.80.3.

(27) Zhao, Y.; Lynch, B. J.; Truhlar, D. G. Doubly Hybrid Meta DFT: New Multi-Coefficient Correlation and Density Functional Methods for Thermochemistry and Thermochemical Kinetics. J. Phys. Chem. A 2004, 108 (21), 4786-4791. https://doi.org/10.1021/jp049253v.

(28) Grimme, S. Semiempirical Hybrid Density Functional with Perturbative Second-Order Correlation. J. Chem. Phys. 2006, 124 (3), 034108. https://doi.org/10.1063/1.2148954.

(29) Görling, A.; Levy, M. Exact Kohn-Sham Scheme Based on Perturbation Theory. Phys. Rev. A 1994, 50 (1), 196-204. https://doi.org/10.1103/PhysRevA.50.196.

(30) Grimme, S. Semiempirical GGA-Type Density Functional Constructed with a LongRange Dispersion Correction. J. Comput. Chem. 2006, 27 (15), 1787-1799. https://doi.org/10.1002/jcc.20495.

(31) Grimme, S.; Antony, J.; Ehrlich, S.; Krieg, H. A Consistent and Accurate Ab Initio Parametrization of Density Functional Dispersion Correction (DFT-D) for the 94 Elements H-Pu. J. Chem. Phys. 2010, 132 (15), 154104. https://doi.org/10.1063/1.3382344.

(32) Grimme, S.; Ehrlich, S.; Goerigk, L. Effect of the Damping Function in Dispersion Corrected Density Functional Theory. J. Comput. Chem. 2011, 32 (7), 1456-1465. https://doi.org/10.1002/jcc.21759.

(33) Grimme, S.; Bannwarth, C.; Caldeweyher, E.; Pisarek, J.; Hansen, A. A General Intermolecular Force Field Based on Tight-Binding Quantum Chemical Calculations. J. 
Chem. Phys. 2017, 147 (16), 161708. https://doi.org/10.1063/1.4991798.

(34) Caldeweyher, E.; Ehlert, S.; Hansen, A.; Neugebauer, H.; Spicher, S.; Bannwarth, C.; Grimme, S. A Generally Applicable Atomic-Charge Dependent London Dispersion Correction. J. Chem. Phys. 2019, 150 (15), 154122. https://doi.org/10.1063/1.5090222.

(35) Vydrov, O. A.; Van Voorhis, T. Nonlocal van Der Waals Density Functional: The Simpler the Better. J. Chem. Phys. 2010, 133 (24), 244103. https://doi.org/10.1063/1.3521275.

(36) Kozuch, S.; Martin, J. M. L. DSD-PBEP86: In Search of the Best Double-Hybrid DFT with Spin-Component Scaled MP2 and Dispersion Corrections. Phys. Chem. Chem. Phys. 2011, 13 (45), 20104. https://doi.org/10.1039/c1cp22592h.

(37) Kozuch, S.; Martin, J. M. L. Spin-Component-Scaled Double Hybrids: An Extensive Search for the Best Fifth-Rung Functionals Blending DFT and Perturbation Theory. $J$. Comput. Chem. 2013, 34 (27), 2327-2344. https://doi.org/10.1002/jcc.23391.

(38) Kozuch, S.; Gruzman, D.; Martin, J. M. L. DSD-BLYP: A General Purpose Double Hybrid Density Functional Including Spin Component Scaling and Dispersion Correction. J. Phys. Chem. C 2010, 114 (48), 20801-20808. https://doi.org/10.1021/jp1070852.

(39) Karton, A.; Tarnopolsky, A.; Lamère, J.-F.; Schatz, G. C.; Martin, J. M. L. Highly Accurate First-Principles Benchmark Data Sets for the Parametrization and Validation of Density Functional and Other Approximate Methods. Derivation of a Robust, Generally Applicable, Double-Hybrid Functional for Thermochemistry and Thermochemical . $J$. Phys. Chem. A 2008, 112 (50), 12868-12886. https://doi.org/10.1021/jp801805p.

(40) Su, N. Q.; Xu, X. The XYG3 Type of Doubly Hybrid Density Functionals. Wiley Interdiscip. Rev. Comput. Mol. Sci. 2016, 6 (6), 721-747. https://doi.org/10.1002/wcms.1274.

(41) Goerigk, L.; Grimme, S. A Thorough Benchmark of Density Functional Methods for General Main Group Thermochemistry, Kinetics, and Noncovalent Interactions. Phys. Chem. Chem. Phys. 2011, 13, 6670-6688. https://doi.org/10.1039/c0cp02984j.

(42) Kesharwani, M. K.; Kozuch, S.; Martin, J. M. L. Comment on "Doubly Hybrid Density Functional XDH-PBE0 from a Parameter-Free Global Hybrid Model PBE0" [J. Chem. Phys. 136, 174103 (2012)]. J. Chem. Phys. 2015, 143 (18), 187101. https://doi.org/10.1063/1.4934819.

(43) Hait, D.; Head-Gordon, M. Communication: XDH Double Hybrid Functionals Can Be Qualitatively Incorrect for Non-Equilibrium Geometries: Dipole Moment Inversion and Barriers to Radical-Radical Association Using XYG3 and XYGJ-OS. J. Chem. Phys. 2018, 148 (17), 171102. https://doi.org/10.1063/1.5031027.

(44) Goerigk, L.; Grimme, S. Double-Hybrid Density Functionals. Wiley Interdiscip. Rev. Comput. Mol. Sci. 2014, 4 (6), 576-600. https://doi.org/10.1002/wcms.1193.

(45) Sancho-García, J. C.; Adamo, C. Double-Hybrid Density Functionals: Merging Wavefunction and Density Approaches to Get the Best of Both Worlds. Phys. Chem. Chem. Phys. 2013, 15 (35), 14581. https://doi.org/10.1039/c3cp50907a.

(46) Su, N. Q.; Zhu, Z.; Xu, X. Doubly Hybrid Density Functionals That Correctly Describe Both Density and Energy for Atoms. Proc. Natl. Acad. Sci. U. S. A. 2018, 115 (10), 22872292. https://doi.org/10.1073/pnas.1713047115.

(47) Brémond, E.; Ciofini, I.; Sancho-García, J. C.; Adamo, C. Nonempirical Double-Hybrid Functionals: An Effective Tool for Chemists. Acc. Chem. Res. 2016, 49 (8), 1503-1513. https://doi.org/10.1021/acs.accounts.6b00232.

(48) Benighaus, T.; Distasio, R.; Lochan, R.; Chai, J.-D.; Head-Gordon, M. Semiempirical 
Double-Hybrid Density Functional with Improved Description of Long-Range Correlation. J. Phys. Chem. A 2008, 112 (12), 2702-2712.

(49) Graham, D.; Menon, A.; Goerigk, L.; Grimme, S.; Radom, L. Optimization and Basis-Set Dependence of a Restricted-Open-Shell Form of B2-PLYP Double-Hybrid Density Functional Theory. J. Phys. Chem. A 2009, 113 (36), 9861-9873.

(50) Chan, B.; Radom, L. Accurate Quadruple- $\zeta$ Basis-Set Approximation for Double-Hybrid Density Functional Theory with an Order of Magnitude Reduction in Computational Cost. Theor. Chem. Acc. 2013, 133 (2), 1426. https://doi.org/10.1007/s00214-013-1426-9.

(51) Mehta, N.; Casanova-Páez, M.; Goerigk, L. Semi-Empirical or Non-Empirical DoubleHybrid Density Functionals: Which Are More Robust? Phys. Chem. Chem. Phys. 2018, 20 (36), 23175-23194. https://doi.org/10.1039/C8CP03852J.

(52) Goerigk, L.; Mehta, N. A Trip to the Density Functional Theory Zoo: Warnings and Recommendations for the User*. Aust. J. Chem. 2019. https://doi.org/10.1071/CH19023.

(53) Mardirossian, N.; Head-Gordon, M. Thirty Years of Density Functional Theory in Computational Chemistry: An Overview and Extensive Assessment of 200 Density Functionals. Mol. Phys. 2017, 115 (19), 2315-2372. https://doi.org/10.1080/00268976.2017.1333644.

(54) Peverati, R.; Truhlar, D. G. Quest for a Universal Density Functional: The Accuracy of Density Functionals across a Broad Spectrum of Databases in Chemistry and Physics. Philos. Trans. R. Soc. A Math. Phys. Eng. Sci. 2014, 372 (2011), 20120476-20120476. https://doi.org/10.1098/rsta.2012.0476.

(55) Goerigk, L.; Hansen, A.; Bauer, C.; Ehrlich, S.; Najibi, A.; Grimme, S. A Look at the Density Functional Theory Zoo with the Advanced GMTKN55 Database for General Main Group Thermochemistry, Kinetics and Noncovalent Interactions. Phys. Chem. Chem. Phys. 2017, 19 (48), 32184-32215. https://doi.org/10.1039/C7CP04913G.

(56) Goerigk, L.; Grimme, S. A General Database for Main Group Thermochemistry, Kinetics, and Noncovalent Interactions - Assessment of Common and Reparameterized ( Meta )GGA Density Functionals. J. Chem. Theory Comput. 2010, 6 (1), 107-126. https://doi.org/10.1021/ct900489g.

(57) Yu, H. S.; Zhang, W.; Verma, P.; He, X.; Truhlar, D. G. Nonseparable ExchangeCorrelation Functional for Molecules, Including Homogeneous Catalysis Involving Transition Metals. Phys. Chem. Chem. Phys. 2015, 17 (18), 12146-12160. https://doi.org/10.1039/C5CP01425E.

(58) Jurecka, P.; Sponer, J.; Cerný, J.; Hobza, P. Benchmark Database of Accurate (MP2 and CCSD(T) Complete Basis Set Limit) Interaction Energies of Small Model Complexes, DNA Base Pairs, and Amino Acid Pairs. Phys. Chem. Chem. Phys. 2006, 8 (17), 19851993. https://doi.org/10.1039/b600027d.

(59) Rezáč, J.; Riley, K. E.; Hobza, P. S66: A Well-Balanced Database of Benchmark Interaction Energies Relevant to Biomolecular Structures. J. Chem. Theory Comput. 2011, 7 (8), 2427-2438. https://doi.org/10.1021/ct2002946.

(60) Řezáč, J.; Riley, K. E.; Hobza, P. Benchmark Calculations of Noncovalent Interactions of Halogenated Molecules. J. Chem. Theory Comput. 2012, 8 (11), 4285-4292. https://doi.org/10.1021/ct300647k.

(61) Hobza, P. Calculations on Noncovalent Interactions and Databases of Benchmark Interaction Energies. Acc. Chem. Res. 2012, 45 (4), 663-672. https://doi.org/10.1021/ar200255p. 
(62) Karton, A.; Daon, S.; Martin, J. M. L. W4-11: A High-Confidence Benchmark Dataset for Computational Thermochemistry Derived from First-Principles W4 Data. Chem. Phys. Lett. 2011, 510, 165-178. https://doi.org/10.1016/j.cplett.2011.05.007.

(63) Kesharwani, M. K.; Manna, D.; Sylvetsky, N.; Martin, J. M. L. The X40×10 Halogen Bonding Benchmark Revisited: Surprising Importance of $(\mathrm{n}-1) \mathrm{d}$ Subvalence Correlation. J. Phys. Chem. A 2018, 122 (8), 2184-2197. https://doi.org/10.1021/acs.jpca.7b10958.

(64) Fogueri, U. R.; Kozuch, S.; Karton, A.; Martin, J. M. L. The Melatonin Conformer Space: Benchmark and Assessment of Wave Function and DFT Methods for a Paradigmatic Biological and Pharmacological Molecule. J. Phys. Chem. A 2013, 117 (10), 2269-2277. https://doi.org/10.1021/jp312644t.

(65) Kozuch, S.; Martin, J. M. L. Halogen Bonds: Benchmarks and Theoretical Analysis. J. Chem. Theory Comput. 2013, 9, 1918-1931. https://doi.org/10.1021/ct301064t.

(66) Martin, J. M. L. What Can We Learn About Dispersion from the Conformer Surface of NPentane? J. Phys. Chem. A 2013, 117 (14), 3118-3132. https://doi.org/10.1021/jp401429u.

(67) Kozuch, S.; Bachrach, S. M.; Martin, J. M. L. Conformational Equilibria in Butane-1,4Diol: A Benchmark of a Prototypical System with Strong Intramolecular H-Bonds. $J$. Phys. Chem. A 2014, 118 (1), 293-303. https://doi.org/10.1021/jp410723v.

(68) Karton, A.; Schreiner, P. R.; Martin, J. M. L. Heats of Formation of Platonic Hydrocarbon Cages by Means of High-Level Thermochemical Procedures. J. Comput. Chem. 2016, 37 (1), 49-58. https://doi.org/10.1002/jcc.23963.

(69) Kesharwani, M. K.; Karton, A.; Martin, J. M. L. Benchmark Ab Initio Conformational Energies for the Proteinogenic Amino Acids through Explicitly Correlated Methods. Assessment of Density Functional Methods. J. Chem. Theory Comput. 2016, 12 (1), 444 454. https://doi.org/10.1021/acs.jctc.5b01066.

(70) Manna, D.; Martin, J. M. L. What Are the Ground State Structures of C 20 and C 24 ? An Explicitly Correlated Ab Initio Approach. J. Phys. Chem. A 2016, 120 (1), 153-160. https://doi.org/10.1021/acs.jpca.5b10266.

(71) Brauer, B.; Kesharwani, M. K.; Kozuch, S.; Martin, J. M. L. The S66x8 Benchmark for Noncovalent Interactions Revisited: Explicitly Correlated Ab Initio Methods and Density Functional Theory. Phys. Chem. Chem. Phys. 2016, 18 (31), 20905-20925. https://doi.org/10.1039/C6CP00688D.

(72) Manna, D.; Kesharwani, M. K.; Sylvetsky, N.; Martin, J. M. L. Conventional and Explicitly Correlated Ab Initio Benchmark Study on Water Clusters: Revision of the BEGDB and WATER27 Data Sets. J. Chem. Theory Comput. 2017, 13 (7), 3136-3152. https://doi.org/10.1021/acs.jctc.6b01046.

(73) Karton, A.; Sylvetsky, N.; Martin, J. M. L. W4-17: A Diverse and High-Confidence Dataset of Atomization Energies for Benchmarking High-Level Electronic Structure Methods. J. Comput. Chem. 2017, 38 (24), 2063-2075. https://doi.org/10.1002/jcc.24854.

(74) Lynch, B. J.; Truhlar, D. G. Small Representative Benchmarks for Thermochemical Calculations. J. Phys. Chem. A 2003, 107 (42), 8996-8999. https://doi.org/10.1021/jp035287b.

(75) Chan, B. Formulation of Small Test Sets Using Large Test Sets for Efficient Assessment of Quantum Chemistry Methods. J. Chem. Theory Comput. 2018, 14 (8), 4254-4262. https://doi.org/10.1021/acs.jctc.8b00514.

(76) Gould, T. 'Diet GMTKN55' Offers Accelerated Benchmarking through a Representative Subset Approach. Phys. Chem. Chem. Phys. 2018, 20 (44), 27735-27739. 
https://doi.org/10.1039/C8CP05554H.

(77) Morgante, P.; Peverati, R. ACCDB: A Collection of Chemistry Databases for Broad Computational Purposes. J. Comput. Chem. 2019, 40 (6), 839-848.

https://doi.org/10.1002/jcc.25761.

(78) Koster, J.; Rahmann, S. Snakemake--a Scalable Bioinformatics Workflow Engine. Bioinformatics 2012, 28 (19), 2520-2522. https://doi.org/10.1093/bioinformatics/bts480.

(79) Huber, P. J.; Ronchetti, E. M. Robust Statistics; Wiley Series in Probability and Statistics; John Wiley \& Sons, Inc.: Hoboken, NJ, USA, 2009. https://doi.org/10.1002/9780470434697.

(80) Weigend, F.; Ahlrichs, R. Balanced Basis Sets of Split Valence, Triple Zeta Valence and Quadruple Zeta Valence Quality for H to Rn: Design and Assessment of Accuracy. Phys. Chem. Chem. Phys. 2005, 7 (18), 3297-3305. https://doi.org/10.1039/b508541a.

(81) Rappoport, D.; Furche, F. Property-Optimized Gaussian Basis Sets for Molecular Response Calculations. J. Chem. Phys. 2010, 133 (13), 134105. https://doi.org/10.1063/1.3484283.

(82) Shao, Y.; Gan, Z.; Epifanovsky, E.; Gilbert, A. T. B.; Wormit, M.; Kussmann, J.; Lange, A. W.; Behn, A.; Deng, J.; Feng, X.; et al. Advances in Molecular Quantum Chemistry Contained in the Q-Chem 4 Program Package. Mol. Phys. 2015, 113 (2), 184-215. https://doi.org/10.1080/00268976.2014.952696.

(83) Dasgupta, S.; Herbert, J. M. Standard Grids for High-Precision Integration of Modern Density Functionals: SG-2 and SG-3. J. Comput. Chem. 2017, 38 (12), 869-882. https://doi.org/10.1002/jcc.24761.

(84) Gill, P. M. .; Johnson, B. G.; Pople, J. A. A Standard Grid for Density Functional Calculations. Chem. Phys. Lett. 1993, 209 (5-6), 506-512. https://doi.org/10.1016/00092614(93)80125-9.

(85) Murray, C.; Handy, N.; Laming, G. Quadrature Schemes for Integrals of Density Functional Theory. Mol. Phys. 1993, 78 (4), 997-1014. https://doi.org/10.1080/00268979300100651.

(86) Lebedev, V. I.; Laikov, D. N. A Quadrature Formula for the Sphere of the 131st Algebraic Order of Accuracy. Dokl. Math. 1999, 59 (3), 477-481.

(87) Brandenburg, J. G.; Bates, J. E.; Sun, J.; Perdew, J. P. Benchmark Tests of a Strongly Constrained Semilocal Functional with a Long-Range Dispersion Correction. Phys. Rev. B - Condens. Matter Mater. Phys. 2016, 94 (11), 17-19. https://doi.org/10.1103/PhysRevB.94.115144.

(88) Chai, J.-D.; Head-Gordon, M. Long-Range Corrected Double-Hybrid Density Functionals. J. Chem. Phys. 2009, 131 (17), 174105. https://doi.org/10.1063/1.3244209.

(89) Sullivan, M. B.; Iron, M. A.; Redfern, P. C.; Martin, J. M. L.; Curtiss, L. A.; Radom, L. Heats of Formation of Alkali Metal and Alkaline Earth Metal Oxides and Hydroxides: Surprisingly Demanding Targets for High-Level Ab Initio Procedures. J. Phys. Chem. A 2003, 107 (29), 5617-5630. https://doi.org/10.1021/jp034851f.

(90) Haworth, N. L.; Sullivan, M. B.; Wilson, A. K.; Martin, J. M. L.; Radom, L. Structures and Thermochemistry of Calcium-Containing Molecules. J. Phys. Chem. A 2005, 109 (40), 9156-9168. https://doi.org/10.1021/jp052889h.

(91) Zhang, Y.; Yang, W. Comment on "Generalized Gradient Approximation Made Simple." Phys. Rev. Lett. 1998, 80 (4), 890-890. https://doi.org/10.1103/PhysRevLett.80.890.

(92) Perdew, J.; Ruzsinszky, A.; Csonka, G.; Constantin, L.; Sun, J. Workhorse Semilocal 
Density Functional for Condensed Matter Physics and Quantum Chemistry. Phys. Rev. Lett. 2009, 103 (2). https://doi.org/10.1103/PhysRevLett.103.026403.

(93) Stephens, P. J.; Devlin, F. J.; Chabalowski, C. F.; Frisch, M. J. Ab Initio Calculation of Vibrational Absorption and Circular Dichroism Spectra Using Density Functional Force Fields. J. Phys. Chem. 1994, 98 (45), 11623-11627. https://doi.org/10.1021/j100096a001.

(94) Adamo, C.; Barone, V. Toward Reliable Density Functional Methods without Adjustable Parameters: The PBE0 Model. J. Chem. Phys. 1999, 110 (13), 6158-6170. https://doi.org/10.1063/1.478522.

(95) Zhao, Y.; Truhlar, D. G. Design of Density Functionals That Are Broadly Accurate for Thermochemistry, Thermochemical Kinetics, and Nonbonded Interactions. J. Phys. Chem. A 2005, 109 (25), 5656-5667. https://doi.org/10.1021/jp050536c.

(96) Zhao, Y.; Truhlar, D. G. The M06 Suite of Density Functionals for Main Group Thermochemistry, Thermochemical Kinetics, Noncovalent Interactions, Excited States, and Transition Elements: Two New Functionals and Systematic Testing of Four M06Class Functionals and 12 Other Function. Theor. Chem. Acc. 2008, 120 (1-3), 215-241. https://doi.org/10.1007/s00214-007-0310-x.

(97) Hui, K.; Chai, J.-D. SCAN-Based Hybrid and Double-Hybrid Density Functionals from Models without Fitted Parameters. J. Chem. Phys. 2016, 144 (4), 044114. https://doi.org/10.1063/1.4940734.

(98) Powell, M. J. D. The BOBYQA Algorithm for Bound Constrained Optimization without Derivatives (DAMPT Report 2009/NA06); Department of Applied Mathematics and Theoretical Physics, University of Cambridge, UK, 2009. https://doi.org/10.1.1.443.7693.

(99) Goerigk, L. A Comprehensive Overview of the DFT-D3 London-Dispersion Correction. In Non-Covalent Interactions in Quantum Chemistry and Physics; Elsevier, 2017; pp 195219. https://doi.org/10.1016/B978-0-12-809835-6.00007-4.

(100) Najibi, A.; Goerigk, L. The Nonlocal Kernel in van Der Waals Density Functionals as an Additive Correction: An Extensive Analysis with Special Emphasis on the B97M-V and SB97M-V Approaches. J. Chem. Theory Comput. 2018, 14 (11), 5725-5738. https://doi.org/10.1021/acs.jctc.8b00842.

(101) Toulouse, J.; Sharkas, K.; Brémond, E.; Adamo, C. Communication : Rationale for a New Class of Double-Hybrid Approximations in Density-Functional Theory. J. Chem. Phys. 2011, 135, 101102. https://doi.org/10.1063/1.3640019.

(102) Sharkas, K.; Toulouse, J.; Savin, A. Double-Hybrid Density-Functional Theory Made Rigorous. J. Chem. Phys. 2011, 134 (6), 064113. https://doi.org/10.1063/1.3544215.

(103) Sancho-García, J. C.; Pérez-Jiménez, A. J.; Savarese, M.; Brémond, E.; Adamo, C. Importance of Orbital Optimization for Double-Hybrid Density Functionals: Application of the OO-PBE-QIDH Model for Closed- and Open-Shell Systems. J. Phys. Chem. A 2016, 120 (10), 1756-1762. https://doi.org/10.1021/acs.jpca.6b00994.

(104) Austin, A.; Petersson, G. A.; Frisch, M. J.; Dobek, F. J.; Scalmani, G.; Throssell, K. A Density Functional with Spherical Atom Dispersion Terms. J. Chem. Theory Comput. 2012, 8 (12), 4989-5007.

(105) Hujo, W.; Grimme, S. Performance of the van Der Waals Density Functional VV10 and (Hybrid)GGA Variants for Thermochemistry and Noncovalent Interactions. J. Chem. Theory Comput. 2011, 7 (12), 3866-3871. https://doi.org/10.1021/ct200644w.

(106) Yu, F. Spin-Component-Scaled Double-Hybrid Density Functionals with Nonlocal van Der Waals Correlations for Noncovalent Interactions. J. Chem. Theory Comput. 2014, 10, 
4400-4407. https://doi.org/10.1021/ct500642x.

(107) Řezáč, J.; Riley, K. E.; Hobza, P. Extensions of the S66 Data Set: More Accurate

Interaction Energies and Angular-Displaced Nonequilibrium Geometries. J. Chem. Theory

Comput. 2011, 7 (11), 3466-3470. https://doi.org/10.1021/ct200523a.

(108) Almlöf, J. Elimination of Energy Denominators in Møller-Plesset Perturbation Theory by a Laplace Transform Approach. Chem. Phys. Lett. 1991, 181 (4), 319-320.

https://doi.org/10.1016/0009-2614(91)80078-C.

(109) Häser, M. Møller-Plesset (MP2) Perturbation Theory for Large Molecules. Theor. Chim. Acta 1993, 87 (1-2), 147-173. https://doi.org/10.1007/BF01113535.

(110) Häser, M.; Almlöf, J. Laplace Transform Techniques in Møller-Plesset Perturbation Theory. J. Chem. Phys. 1992, 96 (1), 489-494. https://doi.org/10.1063/1.462485.

(111) Song, C.; Martínez, T. J. Atomic Orbital-Based SOS-MP2 with Tensor Hypercontraction. I. GPU-Based Tensor Construction and Exploiting Sparsity. J. Chem. Phys. 2016, 144 (17), 174111. https://doi.org/10.1063/1.4948438.

(112) Miehlich, B.; Savin, A.; Stoll, H.; Preuss, H. Results Obtained with the Correlation Energy Density Functionals of Becke and Lee, Yang and Parr. Chem. Phys. Lett. 1989, 157 (3), 200-206. https://doi.org/10.1016/0009-2614(89)87234-3.

(113) Frisch, M. J.; Trucks, G. W.; Schlegel, H. B.; Scuseria, G. E.; Robb, M. A.; Cheeseman, J. R.; Scalmani, G.; Barone, V.; Petersson, G. A.; Nakatsuji, H.; et al. Gaussian 16 Rev. B01. Gaussian, Inc.: Wallingford, CT 2016.

(114) Neese, F. The ORCA Program System. Wiley Interdiscip. Rev. Comput. Mol. Sci. 2012, 2 (1), 73-78. https://doi.org/10.1002/wcms.81.

(115) Neese, F. Software Update: The ORCA Program System, Version 4.0. Wiley Interdiscip. Rev. Comput. Mol. Sci. 2018, 8 (1), 4-9. https://doi.org/10.1002/wcms.1327.

(116) Ghasemi, S. A.; Hofstetter, A.; Saha, S.; Goedecker, S. Interatomic Potentials for Ionic Systems with Density Functional Accuracy Based on Charge Densities Obtained by a Neural Network. Phys. Rev. B - Condens. Matter Mater. Phys. 2015, 92 (4), 1-6. https://doi.org/10.1103/PhysRevB.92.045131.

(117) Rappé, A. K.; Goddard, W. A. Charge Equilibration for Molecular Dynamics Simulations. J. Phys. Chem. 1991, 95 (8), 3358-3363. https://doi.org/10.1021/j100161a070.

(118) Verstraelen, T.; Van Speybroeck, V.; Waroquier, M. The Electronegativity Equalization Method and the Split Charge Equilibration Applied to Organic Systems: Parametrization, Validation, and Comparison. J. Chem. Phys. 2009, 131 (4), 044127. https://doi.org/10.1063/1.3187034.

(119) Korth, M.; Grimme, S. "Mindless" DFT Benchmarking. J. Chem. Theory Comput. 2009, 5 (4), 993-1003. https://doi.org/10.1021/ct800511q.

(120) Iron, M. A.; Janes, T. Evaluating Transition Metal Barrier Heights with the Latest Density Functional Theory Exchange-Correlation Functionals: The MOBH35 Benchmark Database. J. Phys. Chem. A 2019, 123 (17), 3761-3781. https://doi.org/10.1021/acs.jpca.9b01546.

(121) Dohm, S.; Hansen, A.; Steinmetz, M.; Grimme, S.; Checinski, M. P. Comprehensive Thermochemical Benchmark Set of Realistic Closed-Shell Metal Organic Reactions. $J$. Chem. Theory Comput. 2018, 14 (5), 2596-2608. https://doi.org/10.1021/acs.jctc.7b01183.

(122) Goerigk, L.; Grimme, S. Efficient and Accurate Double-Hybrid-Meta-GGA Density Functionals-Evaluation with the Extended GMTKN30 Database for General Main 
Group Thermochemistry, Kinetics, and Noncovalent Interactions. J. Chem. Theory

Comput. 2011, 7 (2), 291-309. https://doi.org/10.1021/ct100466k.

(123) Mezei, P. D.; Csonka, G. I.; Ruzsinszky, A.; Kállay, M. Construction and Application of a New Dual-Hybrid Random Phase Approximation. J. Chem. Theory Comput. 2015, 11 (10), 4615-4626. https://doi.org/10.1021/acs.jctc.5b00420.

(124) Mezei, P. D.; Csonka, G. I.; Ruzsinszky, A.; Kállay, M. Construction of a SpinComponent Scaled Dual-Hybrid Random Phase Approximation. J. Chem. Theory Comput. 2017, 13 (2), 796-803. https://doi.org/10.1021/acs.jctc.6b01140.

(125) Chan, B.; Goerigk, L.; Radom, L. On the Inclusion of Post-MP2 Contributions to DoubleHybrid Density Functionals. J. Comput. Chem. 2016, 37 (2), 183-193. https://doi.org/10.1002/jcc.23972.

(126) Kesharwani, M. K.; Brauer, B.; Martin, J. M. L. Frequency and Zero-Point Vibrational Energy Scale Factors for Double-Hybrid Density Functionals (and Other Selected Methods): Can Anharmonic Force Fields Be Avoided? J. Phys. Chem. A 2015, 119, 17011714. https://doi.org/10.1021/jp508422u.

(127) Efremenko, I.; Martin, J. M. L. Coupled Cluster Benchmark of New Density Functionals and Domain Pair Natural Orbital Methods: Mechanisms of Hydroarylation and Oxidative Coupling Catalyzed by Ru(II) Chloride Carbonyls. 2019.

(128) Weissman, H.; Song, X.; Milstein, D. Ru-Catalyzed Oxidative Coupling of Arenes with Olefins Using O 2. J. Am. Chem. Soc. 2001, 123 (2), 337-338. https://doi.org/10.1021/ja003361n.

(129) Perdew, J. P. Density-Functional Approximation for the Correlation Energy of the Inhomogeneous Electron Gas. Phys. Rev. B 1986, 33 (12), 8822-8824. https://doi.org/10.1103/PhysRevB.33.8822.

(130) Lee, C.; Yang, W.; Parr, R. G. Development of the Colle-Salvetti Correlation-Energy Formula into a Functional of the Electron Density. Phys. Rev. B 1988, 37 (2), 785-789. https://doi.org/10.1103/PhysRevB.37.785. 
Table of Contents Graphic

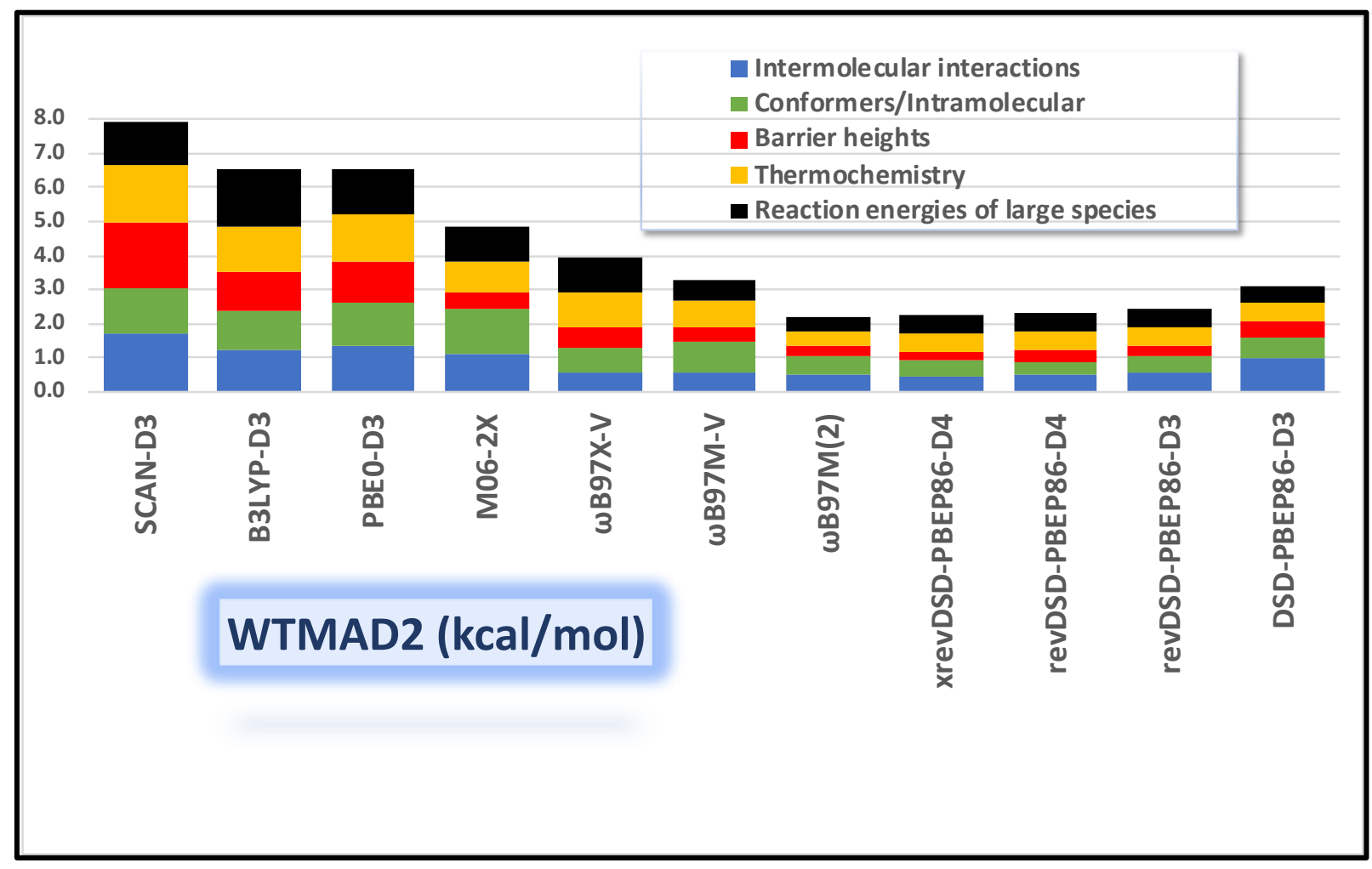




\title{
Electronic Supporting Information
}

\section{Minimally Empirical Double Hybrid Functionals Trained Against the GMTKN55 \\ Database: revDSD-PBEP86-D4, revDOD-PBE-D4, and DOD-SCAN-D4}

\author{
Golokesh Santra, Nitai Sylvetsky, and Jan M.L. Martin
}

Department of Organic Chemistry, Weizmann Institute of Science, 7610001 Rehovot, Israel.

Email:gershom@weizmann.ac.il 
Table S1: Abbreviations used and their descriptions

\begin{tabular}{|c|c|}
\hline Abbreviation & Description \\
\hline $\mathrm{ACONF}^{1}$ & Relative energies of alkane conformers \\
\hline $\mathrm{ADIM}^{2}$ & Interaction energies of $n$-alkane dimers \\
\hline $\mathrm{AHB} 21^{3}$ & Interaction energies in anion-neutral dimers \\
\hline AL2X $6^{4}$ & Dimerisation energies of $\mathrm{AlX} 3$ compounds \\
\hline $\mathrm{ALK}^{4}$ & Dissociation and other reactions of alkaline compounds \\
\hline ALKBDE10 $10^{5}$ & Dissociation energies in group- 1 and -2 diatomics \\
\hline AMINO20X46 ${ }^{6}$ & Relative energies in amino acid conformers \\
\hline $\mathrm{BH}_{76 \mathrm{RC}^{7}}$ & $\begin{array}{l}\text { Barrier heights of hydrogen transfer, heavy atom transfer, nucleophilic substitution, unimolecular and } \\
\text { association reactions }\end{array}$ \\
\hline $\mathrm{BH} 76^{8,9,7}$ & Reaction energies of the $\mathrm{BH} 7610,11,23$ set \\
\hline BHDIV $10^{4}$ & Diverse reaction barrier heights \\
\hline BHPERI $^{4,10,11,12}$ & Barrier heights of pericyclic reactions \\
\hline $\mathrm{BHROT}_{2} 7^{4}$ & Barrier heights for rotation around single bonds \\
\hline BSR36 $6^{13,14}$ & Bond-separation reactions of saturated hydrocarbons \\
\hline BUT14DIOL $^{15}$ & Relative energies in butane-1,4-diol conformers \\
\hline${\mathrm{C} 60 \mathrm{ISO}^{16}}^{16}$ & Relative energies between C60 isomers \\
\hline $\mathrm{CARBHB} 2^{4}$ & Hydrogen-bonded complexes between carbene analogues and $\mathrm{H} 2 \mathrm{O}, \mathrm{NH} 3$, or $\mathrm{HCl}$ \\
\hline $\mathrm{CDIE}_{20} 0^{17}$ & Double-bond isomerisation energies in cyclic systems \\
\hline $\mathrm{CHB}^{3}$ & Interaction energies in cation-neutral dimers \\
\hline $\mathrm{DARC}^{7,18}$ & Reaction energies of Diels-Alder reactions \\
\hline DC13 $3^{19,7,20,21-29}$ & 13 difficult cases for DFT methods \\
\hline DIPCS10 $0^{4}$ & Double-ionisation potentials of closed-shell systems \\
\hline FH51 30,31 & Reaction energies in various (in-)organic systems \\
\hline $\mathrm{G}^{21 \mathrm{EA}^{7,32}}$ & Adiabatic electron affinities \\
\hline $\mathrm{G} 21 \mathrm{IP}^{7,32}$ & Adiabatic ionisation potentials \\
\hline $\mathrm{G} 2 \mathrm{RC}^{7,33}$ & Reaction energies of selected G2/97 systems \\
\hline HAL5 $9^{34,35}$ & Binding energies in halogenated dimers (incl. halogen bonds) \\
\hline HEAVY289 & Noncovalent interaction energies between heavy element hydrides \\
\hline HEAVYSB1 $1^{4}$ & Dissociation energies in heavy-element compounds \\
\hline $\mathrm{ICONF}^{4}$ & Relative energies in conformers of inorganic systems \\
\hline IDISP $^{7,36-39}$ & Intramolecular dispersion interactions \\
\hline IL16 $^{3}$ & Interaction energies in anion-cation dimers \\
\hline INV24 40 & Inversion/racemisation barrier heights \\
\hline ISO3436 & Isomerisation energies of small and medium-sized organic molecules \\
\hline ISOL$_{24}^{41}$ & Isomerisation energies of large organic molecules \\
\hline MB16-43 & Decomposition energies of artificial molecules \\
\hline $\mathrm{MCONF}^{42}$ & Relative energies in melatonin conformers \\
\hline $\mathrm{NBPRC}^{7,38,43}$ & $\begin{array}{l}\text { Oligomerisations and } \mathrm{H} 2 \text { fragmentations of } \mathrm{NH} 3 / \mathrm{BH} 3 \text { systems; } \mathrm{H} 2 \text { activation reactions with } \\
\mathrm{PH} 3 / \mathrm{BH} 3 \text { systems }\end{array}$ \\
\hline${\mathrm{PA} 26^{4}}^{4}$ & Adiabatic proton affinities (incl. of amino acids) \\
\hline PArel $^{4}$ & Relative energies in protonated isomers \\
\hline PCONF21 & Relative energies in tri- and tetrapeptide conformers \\
\hline $\mathrm{PNICO} 23^{44}$ & Interaction energies in pnicogen-containing dimers \\
\hline PX13 45 & Proton-exchange barriers in $\mathrm{H} 2 \mathrm{O}, \mathrm{NH} 3$, and HF clusters \\
\hline $\mathrm{RC} 21^{4}$ & Fragmentations and rearrangements in radical cations \\
\hline $\mathrm{RG}^{2} 8^{4}$ & Interaction energies in rare-gas complexes \\
\hline $\mathrm{RSE}_{4} 3^{46}$ & Radical-stabilisation energies \\
\hline $\mathrm{S} 22^{47}$ & Binding energies of noncovalently bound dimers \\
\hline S66 $6^{48}$ & Binding energies of noncovalently bound dimers \\
\hline $\mathrm{SCONF}^{7,49}$ & Relative energies of sugar conformers \\
\hline SIE4X $4^{50}$ & Self-interaction-error related problems \\
\hline TAUT15 $5^{4}$ & Relative energies in tautomers \\
\hline UPU23 $3^{51}$ & Relative energies between RNA-backbone conformers \\
\hline $\mathrm{W} 4-11^{52}$ & Total atomisation energies \\
\hline
\end{tabular}




\begin{tabular}{|c|c|}
\hline WATER27 $7^{53}$ & Binding energies in $(\mathrm{H} 2 \mathrm{O}) n, \mathrm{H}+(\mathrm{H} 2 \mathrm{O}) \mathrm{n}$ and $\mathrm{OH}-(\mathrm{H} 2 \mathrm{O}) \mathrm{n}$ \\
\hline WCPT $18^{54}$ & Proton-transfer barriers in uncatalysed and water-catalysed reactions \\
\hline YBDE18 $18^{55}$ & Bond-dissociation energies in ylides \\
\hline
\end{tabular}

Table S2: A breakdown by components of DSD-SCAN $50-\mathrm{D} 3(\mathrm{BJ})$ taking $\mathrm{C}_{\mathrm{x}, \mathrm{HF}}=8^{-1 / 3}=0.5$

\begin{tabular}{|c|c|c|c|c|c|c|c|c|c|}
\hline $\begin{array}{l}\text { Serial } \\
\text { No. }\end{array}$ & Type & Set Name & $\begin{array}{l}\text { No. of } \\
\text { Points }\end{array}$ & MAD & MSD & RSMD & $\overline{\left|\Delta E_{\text {ref }}\right|}$ & $\triangle$ WTMAD2 & 5MAD/4RMSD \\
\hline 1 & Conformer & ACONF & 15 & 0.109 & 0.109 & 0.127 & 1.833 & 0.034 & 1.081 \\
\hline 2 & Intermol & ADIM6 & 6 & 0.583 & -0.583 & 0.629 & 3.358 & 0.040 & 1.158 \\
\hline 3 & Intermol & AHB21 & 21 & 0.347 & -0.290 & 0.497 & 22.486 & 0.012 & 0.874 \\
\hline 4 & Thermo & AL2X6 & 6 & 0.620 & 0.620 & 0.659 & 35.883 & 0.004 & 1.176 \\
\hline 5 & Thermo & ALK8 & 8 & 1.466 & 1.371 & 2.209 & 62.601 & 0.007 & 0.829 \\
\hline 6 & Thermo & ALKBDE10 & 10 & 3.596 & -2.447 & 4.258 & 100.690 & 0.014 & 1.056 \\
\hline 7 & Conformer & AMINO20X4 & 80 & 0.128 & -0.013 & 0.160 & 2.439 & 0.159 & 0.997 \\
\hline 8 & Barrier & BH76RC & 30 & 1.698 & -0.110 & 2.109 & 21.392 & 0.090 & 1.007 \\
\hline 9 & Thermo & BH76 & 76 & 1.956 & -1.671 & 2.346 & 18.614 & 0.303 & 1.042 \\
\hline 10 & Barrier & BHDIV10 & 10 & 1.859 & -1.437 & 2.068 & 45.333 & 0.016 & 1.124 \\
\hline 11 & Barrier & BHPERI & 26 & 1.595 & -1.533 & 1.724 & 20.873 & 0.075 & 1.156 \\
\hline 12 & Barrier & BHROT27 & 27 & 0.320 & 0.314 & 0.427 & 6.273 & 0.052 & 0.935 \\
\hline 13 & REACLarge & BSR36 & 36 & 1.288 & -1.288 & 1.395 & 16.197 & 0.109 & 1.154 \\
\hline 14 & Conformer & BUT14DIOL & 64 & 0.045 & -0.036 & 0.051 & 2.800 & 0.039 & 1.105 \\
\hline 15 & REACLarge & C60ISO & 9 & 6.204 & -6.100 & 8.289 & 98.252 & 0.022 & 0.936 \\
\hline 16 & Intermol & CARBHB12 & 12 & 0.404 & 0.391 & 0.606 & 6.036 & 0.030 & 0.833 \\
\hline 17 & REACLarge & CDIE20 & 20 & 0.564 & 0.564 & 0.632 & 4.055 & 0.106 & 1.115 \\
\hline 18 & Intermol & CHB6 & 6 & 0.822 & -0.822 & 1.008 & 26.785 & 0.007 & 1.019 \\
\hline 19 & REACLarge & DARC & 14 & 0.780 & -0.348 & 0.975 & 32.471 & 0.013 & 1.001 \\
\hline 20 & Thermo & DC13 & 13 & 2.505 & 0.465 & 3.806 & 54.978 & 0.023 & 0.823 \\
\hline 21 & Thermo & DIPCS10 & 10 & 5.008 & -5.008 & 5.666 & 654.260 & 0.003 & 1.105 \\
\hline 22 & Thermo & FH51 & 51 & 1.048 & -0.150 & 1.479 & 31.011 & 0.065 & 0.886 \\
\hline 23 & Thermo & G21EA & 25 & 3.372 & -3.091 & 3.875 & 33.624 & 0.095 & 1.088 \\
\hline 24 & Thermo & G21IP & 36 & 2.815 & -1.584 & 3.498 & 257.610 & 0.015 & 1.006 \\
\hline 25 & Thermo & $\mathrm{G} 2 \mathrm{RC}$ & 25 & 2.671 & 0.166 & 3.374 & 51.263 & 0.049 & 0.990 \\
\hline 26 & Intermol & HAL59 & 59 & 0.353 & 0.237 & 0.544 & 4.592 & 0.172 & 0.812 \\
\hline 27 & Intermol & HEAVY28 & 28 & 0.121 & -0.052 & 0.150 & 1.241 & 0.103 & 1.008 \\
\hline 28 & Thermo & HEAVYSB11 & 11 & 1.628 & -1.623 & 2.045 & 58.023 & 0.012 & 0.995 \\
\hline 29 & Conformer & ICONF & 17 & 0.106 & 0.036 & 0.132 & 3.266 & 0.021 & 1.005 \\
\hline 30 & Conformer & IDISP & 6 & 1.007 & 0.658 & 1.177 & 14.223 & 0.016 & 1.069 \\
\hline 31 & Intermol & IL16 & 16 & 0.402 & 0.402 & 0.451 & 109.045 & 0.002 & 1.114 \\
\hline 32 & Barrier & INV24 & 24 & 0.654 & 0.180 & 1.027 & 31.846 & 0.019 & 0.797 \\
\hline 33 & REACLarge & ISO34 & 34 & 0.574 & -0.196 & 0.782 & 14.570 & 0.051 & 0.917 \\
\hline 34 & REACLarge & ISOL24 & 24 & 1.504 & -0.289 & 2.145 & 21.919 & 0.063 & 0.877 \\
\hline 35 & REACLarge & MB16-43 & 43 & 5.510 & -2.022 & 7.354 & 468.394 & 0.019 & 0.937 \\
\hline 36 & Conformer & MCONF & 51 & 0.238 & 0.187 & 0.285 & 4.971 & 0.093 & 1.046 \\
\hline 37 & Thermo & NBPRC & 6 & 0.888 & -0.734 & 1.053 & 24.683 & 0.008 & 1.055 \\
\hline 38 & Thermo & PA26 & 26 & 2.527 & 2.527 & 2.998 & 189.054 & 0.013 & 1.054 \\
\hline 39 & REACLarge & PAREL & 20 & 0.636 & 0.239 & 1.058 & 4.630 & 0.104 & 0.751 \\
\hline 40 & Conformer & PCONF21 & 18 & 0.217 & 0.113 & 0.277 & 1.622 & 0.091 & 0.979 \\
\hline 41 & Intermol & $\mathrm{PNICO} 23$ & 23 & 0.270 & 0.237 & 0.443 & 4.273 & 0.055 & 0.762 \\
\hline 42 & Barrier & PX13 & 13 & 2.498 & -2.498 & 2.584 & 33.362 & 0.037 & 1.209 \\
\hline 43 & Thermo & $\mathrm{RC} 21$ & 21 & 0.908 & 0.134 & 1.191 & 35.699 & 0.020 & 0.953 \\
\hline 44 & Intermol & RG18 & 18 & 0.068 & -0.028 & 0.096 & 0.580 & 0.080 & 0.889 \\
\hline 45 & REACLarge & RSE43 & 43 & 0.600 & 0.545 & 0.981 & 7.602 & 0.129 & 0.764 \\
\hline
\end{tabular}




\begin{tabular}{|l|l|l|l|r|r|r|r|r|r|}
\hline 46 & Intermol & S22 & 22 & 0.207 & -0.035 & 0.280 & 7.302 & 0.024 & 0.927 \\
\hline 47 & Intermol & S66 & 66 & 0.194 & -0.082 & 0.256 & 5.467 & 0.089 & 0.947 \\
\hline 48 & Conformer & SCONF & 17 & 0.044 & 0.001 & 0.066 & 4.600 & 0.006 & 0.824 \\
\hline 49 & Thermo & SIE4X4 & 16 & 7.141 & 7.141 & 8.362 & 33.725 & 0.129 & 1.067 \\
\hline 50 & Thermo & TAUT15 & 15 & 0.825 & -0.263 & 0.922 & 3.046 & 0.154 & 1.118 \\
\hline 51 & Conformer & UPU23 & 23 & 0.351 & 0.144 & 0.469 & 5.723 & 0.054 & 0.936 \\
\hline 52 & Thermo & W4-11 & 140 & 5.694 & -5.654 & 6.468 & 306.914 & 0.099 & 1.100 \\
\hline 53 & Intermol & WATER27 & 27 & 0.561 & 0.412 & 0.678 & 81.174 & 0.007 & 1.033 \\
\hline 54 & Barrier & WCPT18 & 18 & 1.694 & -1.694 & 2.059 & 34.988 & 0.033 & 1.028 \\
\hline 55 & Thermo & YBDE18 & 18 & 1.573 & -1.136 & 1.927 & 49.276 & 0.022 & 1.021 \\
\hline
\end{tabular}

Note: $5 \mathrm{MAD} / 4 \mathrm{RMSD}$ should be $\approx 1$ for an unbiased normal distribution ${ }^{56,57}$. The $\overline{\left|\boldsymbol{\Delta \boldsymbol { E } _ { \text { ref } }}\right|}$ Column has been omitted in subsequent tables.

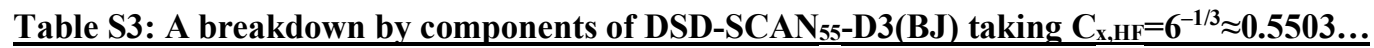

\begin{tabular}{|c|c|c|c|c|c|c|c|c|}
\hline $\begin{array}{c}\text { Serial } \\
\text { No. }\end{array}$ & Type & Set Name & $\begin{array}{l}\text { No. of } \\
\text { Points }\end{array}$ & MAD & MSD & RSMD & AWTMAD2 & 5MAD/4RMSD \\
\hline 1 & Conformer & ACONF & 15 & 0.097 & 0.097 & 0.113 & 0.030 & 1.066 \\
\hline 2 & Intermol & ADIM6 & 6 & 0.591 & -0.591 & 0.636 & 0.040 & 1.161 \\
\hline 3 & Intermol & AHB21 & 21 & 0.334 & -0.263 & 0.470 & 0.012 & 0.888 \\
\hline 4 & Thermo & AL2X6 & 6 & 0.498 & 0.498 & 0.543 & 0.003 & 1.146 \\
\hline 5 & Thermo & ALK8 & 8 & 1.259 & 1.150 & 1.937 & 0.006 & 0.812 \\
\hline 6 & Thermo & ALKBDE10 & 10 & 3.102 & -1.707 & 3.746 & 0.012 & 1.035 \\
\hline 7 & Conformer & AMINO20X4 & 80 & 0.123 & -0.018 & 0.154 & 0.153 & 0.997 \\
\hline 8 & Barrier & BH76RC & 30 & 1.606 & -0.150 & 1.990 & 0.085 & 1.009 \\
\hline 9 & Thermo & BH76 & 76 & 1.714 & -1.201 & 2.146 & 0.265 & 0.998 \\
\hline 10 & Barrier & BHDIV 10 & 10 & 1.610 & -1.256 & 1.824 & 0.014 & 1.104 \\
\hline 11 & Barrier & BHPERI & 26 & 1.520 & -1.444 & 1.647 & 0.072 & 1.154 \\
\hline 12 & Barrier & BHROT27 & 27 & 0.275 & 0.269 & 0.365 & 0.045 & 0.941 \\
\hline 13 & REACLarge & BSR36 & 36 & 1.193 & -1.193 & 1.275 & 0.101 & 1.170 \\
\hline 14 & Conformer & BUT14DIOL & 64 & 0.050 & -0.045 & 0.055 & 0.044 & 1.138 \\
\hline 15 & REACLarge & C60ISO & 9 & 6.549 & -6.468 & 8.693 & 0.023 & 0.942 \\
\hline 16 & Intermol & CARBHB12 & 12 & 0.376 & 0.362 & 0.560 & 0.028 & 0.840 \\
\hline 17 & REACLarge & CDIE20 & 20 & 0.503 & 0.503 & 0.566 & 0.094 & 1.112 \\
\hline 18 & Intermol & CHB6 & 6 & 0.864 & -0.864 & 1.051 & 0.007 & 1.028 \\
\hline 19 & REACLarge & DARC & 14 & 0.893 & -0.704 & 1.070 & 0.015 & 1.044 \\
\hline 20 & Thermo & DC13 & 13 & 2.219 & 0.214 & 3.536 & 0.020 & 0.784 \\
\hline 21 & Thermo & DIPCS10 & 10 & 4.136 & -4.136 & 4.825 & 0.002 & 1.071 \\
\hline 22 & Thermo & FH51 & 51 & 0.972 & -0.193 & 1.341 & 0.061 & 0.906 \\
\hline 23 & Thermo & G21EA & 25 & 2.978 & -2.610 & 3.425 & 0.084 & 1.087 \\
\hline 24 & Thermo & G21IP & 36 & 2.418 & -1.091 & 3.100 & 0.013 & 0.975 \\
\hline 25 & Thermo & G2RC & 25 & 2.445 & 0.123 & 3.085 & 0.045 & 0.991 \\
\hline 26 & Intermol & HAL59 & 59 & 0.323 & 0.203 & 0.489 & 0.157 & 0.826 \\
\hline 27 & Intermol & HEAVY28 & 28 & 0.110 & -0.044 & 0.137 & 0.094 & 1.003 \\
\hline 28 & Thermo & HEAVYSB11 & 11 & 1.221 & -1.081 & 1.582 & 0.009 & 0.965 \\
\hline 29 & Conformer & ICONF & 17 & 0.093 & 0.024 & 0.121 & 0.018 & 0.964 \\
\hline 30 & Conformer & IDISP & 6 & 0.785 & 0.473 & 0.865 & 0.013 & 1.135 \\
\hline 31 & Intermol & IL16 & 16 & 0.395 & 0.395 & 0.437 & 0.002 & 1.131 \\
\hline 32 & Barrier & INV24 & 24 & 0.642 & 0.192 & 1.031 & 0.018 & 0.779 \\
\hline 33 & REACLarge & ISO34 & 34 & 0.539 & -0.200 & 0.690 & 0.048 & 0.977 \\
\hline 34 & REACLarge & ISOL24 & 24 & 1.241 & -0.171 & 1.768 & 0.052 & 0.878 \\
\hline 35 & REACLarge & MB16-43 & 43 & 4.992 & -1.162 & 6.603 & 0.017 & 0.945 \\
\hline 36 & Conformer & MCONF & 51 & 0.245 & 0.196 & 0.288 & 0.095 & 1.066 \\
\hline 37 & Thermo & NBPRC & 6 & 0.831 & -0.674 & 0.980 & 0.008 & 1.060 \\
\hline 38 & Thermo & PA26 & 26 & 2.370 & 2.370 & 2.782 & 0.012 & 1.065 \\
\hline 39 & REACLarge & PAREL & 20 & 0.606 & 0.202 & 0.977 & 0.099 & 0.776 \\
\hline
\end{tabular}




\begin{tabular}{|l|l|l|l|l|l|l|l|l|}
\hline 40 & Conformer & PCONF21 & 18 & 0.215 & 0.110 & 0.275 & 0.091 & 0.978 \\
\hline 41 & Intermol & PNICO23 & 23 & 0.232 & 0.192 & 0.382 & 0.047 & 0.760 \\
\hline 42 & Barrier & PX13 & 13 & 2.361 & -2.361 & 2.452 & 0.035 & 1.204 \\
\hline 43 & Thermo & RC21 & 21 & 0.990 & -0.209 & 1.235 & 0.022 & 1.002 \\
\hline 44 & Intermol & RG18 & 18 & 0.066 & -0.037 & 0.099 & 0.078 & 0.836 \\
\hline 45 & REACLarge & RSE43 & 43 & 0.757 & 0.727 & 1.317 & 0.162 & 0.718 \\
\hline 46 & Intermol & S22 & 22 & 0.212 & -0.042 & 0.282 & 0.024 & 0.938 \\
\hline 47 & Intermol & S66 & 66 & 0.192 & -0.095 & 0.254 & 0.088 & 0.943 \\
\hline 48 & Conformer & SCONF & 17 & 0.039 & 0.000 & 0.059 & 0.006 & 0.827 \\
\hline 49 & Thermo & SIE4X4 & 16 & 6.324 & 6.324 & 7.433 & 0.114 & 1.064 \\
\hline 50 & Thermo & TAUT15 & 15 & 0.760 & -0.288 & 0.864 & 0.142 & 1.099 \\
\hline 51 & Conformer & UPU23 & 23 & 0.354 & 0.153 & 0.472 & 0.054 & 0.937 \\
\hline 52 & Thermo & W4-11 & 140 & 3.663 & -3.401 & 4.437 & 0.063 & 1.032 \\
\hline 53 & Intermol & WATER27 & 27 & 0.435 & 0.191 & 0.572 & 0.006 & 0.951 \\
\hline 54 & Barrier & WCPT18 & 18 & 1.536 & -1.536 & 1.866 & 0.030 & 1.029 \\
\hline 55 & Thermo & YBDE18 & 18 & 1.124 & -0.299 & 1.456 & 0.016 & 0.965 \\
\hline
\end{tabular}

Table S4: A breakdown by components of DSD-SCAN 58 -D3(BJ) taking $\mathrm{C}_{\mathrm{x}, \mathrm{HF}}=5^{-1 / 3} \approx 0.5848 \ldots$

\begin{tabular}{|c|c|c|c|c|c|c|c|c|}
\hline $\begin{array}{c}\text { Serial } \\
\text { No. }\end{array}$ & Type & Set Name & $\begin{array}{l}\text { No. of } \\
\text { Points }\end{array}$ & MAD & MSD & RSMD & AWTMAD2 & 5MAD/4RMSD \\
\hline 1 & Conformer & ACONF & 15 & 0.087 & 0.087 & 0.104 & 0.027 & 1.045 \\
\hline 2 & Intermol & ADIM6 & 6 & 0.591 & -0.591 & 0.636 & 0.040 & 1.161 \\
\hline 3 & Intermol & AHB21 & 21 & 0.329 & -0.253 & 0.457 & 0.012 & 0.900 \\
\hline 4 & Thermo & AL2X6 & 6 & 0.499 & 0.499 & 0.547 & 0.003 & 1.142 \\
\hline 5 & Thermo & ALK8 & 8 & 1.227 & 1.101 & 1.821 & 0.006 & 0.843 \\
\hline 6 & Thermo & ALKBDE10 & 10 & 2.922 & -1.526 & 3.498 & 0.011 & 1.044 \\
\hline 7 & Conformer & AMINO20X4 & 80 & 0.121 & -0.020 & 0.152 & 0.150 & 0.991 \\
\hline 8 & Barrier & BH76RC & 30 & 1.547 & -0.184 & 1.912 & 0.082 & 1.011 \\
\hline 9 & Thermo & BH76 & 76 & 1.544 & -0.819 & 2.068 & 0.239 & 0.933 \\
\hline 10 & Barrier & BHDIV10 & 10 & 1.427 & -1.079 & 1.610 & 0.012 & 1.108 \\
\hline 11 & Barrier & BHPERI & 26 & 1.425 & -1.336 & 1.562 & 0.067 & 1.140 \\
\hline 12 & Barrier & BHROT27 & 27 & 0.254 & 0.248 & 0.334 & 0.041 & 0.949 \\
\hline 13 & REACLarge & BSR36 & 36 & 1.115 & -1.115 & 1.181 & 0.094 & 1.179 \\
\hline 14 & Conformer & BUT14DIOL & 64 & 0.056 & -0.053 & 0.061 & 0.049 & 1.157 \\
\hline 15 & REACLarge & C60ISO & 9 & 6.456 & -6.367 & 8.601 & 0.022 & 0.938 \\
\hline 16 & Intermol & CARBHB12 & 12 & 0.358 & 0.344 & 0.528 & 0.027 & 0.848 \\
\hline 17 & REACLarge & CDIE20 & 20 & 0.455 & 0.455 & 0.517 & 0.085 & 1.100 \\
\hline 18 & Intermol & CHB6 & 6 & 0.918 & -0.918 & 1.106 & 0.008 & 1.038 \\
\hline 19 & REACLarge & DARC & 14 & 1.036 & -1.030 & 1.267 & 0.017 & 1.022 \\
\hline 20 & Thermo & DC13 & 13 & 2.287 & -0.011 & 3.427 & 0.021 & 0.834 \\
\hline 21 & Thermo & DIPCS10 & 10 & 3.747 & -3.747 & 4.473 & 0.002 & 1.047 \\
\hline 22 & Thermo & FH51 & 51 & 0.927 & -0.264 & 1.266 & 0.058 & 0.915 \\
\hline 23 & Thermo & G21EA & 25 & 2.866 & -2.441 & 3.315 & 0.081 & 1.081 \\
\hline 24 & Thermo & G21IP & 36 & 2.287 & -0.868 & 2.974 & 0.012 & 0.961 \\
\hline 25 & Thermo & G2RC & 25 & 2.296 & 0.031 & 2.893 & 0.043 & 0.992 \\
\hline 26 & Intermol & HAL59 & 59 & 0.303 & 0.177 & 0.453 & 0.148 & 0.837 \\
\hline 27 & Intermol & HEAVY28 & 28 & 0.107 & -0.043 & 0.133 & 0.091 & 1.005 \\
\hline 28 & Thermo & HEAVYSB11 & 11 & 1.063 & -0.789 & 1.382 & 0.008 & 0.962 \\
\hline 29 & Conformer & ICONF & 17 & 0.092 & 0.020 & 0.117 & 0.018 & 0.985 \\
\hline 30 & Conformer & IDISP & 6 & 0.611 & 0.330 & 0.746 & 0.010 & 1.025 \\
\hline 31 & Intermol & IL16 & 16 & 0.392 & 0.392 & 0.432 & 0.002 & 1.136 \\
\hline 32 & Barrier & INV24 & 24 & 0.639 & 0.244 & 1.067 & 0.018 & 0.749 \\
\hline 33 & REACLarge & ISO34 & 34 & 0.514 & -0.189 & 0.635 & 0.046 & 1.012 \\
\hline 34 & REACLarge & ISOL24 & 24 & 1.050 & -0.048 & 1.508 & 0.044 & 0.870 \\
\hline 35 & REACLarge & MB16-43 & 43 & 4.826 & -0.392 & 6.347 & 0.017 & 0.950 \\
\hline 36 & Conformer & MCONF & 51 & 0.247 & 0.200 & 0.287 & 0.096 & 1.078 \\
\hline 37 & Thermo & NBPRC & 6 & 0.806 & -0.658 & 0.947 & 0.007 & 1.065 \\
\hline 38 & Thermo & PA26 & 26 & 2.306 & 2.306 & 2.682 & 0.012 & 1.075 \\
\hline 39 & REACLarge & PAREL & 20 & 0.575 & 0.186 & 0.911 & 0.094 & 0.789 \\
\hline
\end{tabular}




\begin{tabular}{|l|l|l|r|r|r|r|r|c|}
\hline 40 & Conformer & PCONF21 & 18 & 0.212 & 0.111 & 0.273 & 0.089 & 0.971 \\
\hline 41 & Intermol & PNICO23 & 23 & 0.216 & 0.170 & 0.350 & 0.044 & 0.771 \\
\hline 42 & Barrier & PX13 & 13 & 2.166 & -2.166 & 2.262 & 0.032 & 1.197 \\
\hline 43 & Thermo & RC21 & 21 & 1.127 & -0.430 & 1.351 & 0.025 & 1.043 \\
\hline 44 & Intermol & RG18 & 18 & 0.069 & -0.043 & 0.104 & 0.082 & 0.834 \\
\hline 45 & REACLarge & RSE43 & 43 & 0.885 & 0.866 & 1.558 & 0.190 & 0.710 \\
\hline 46 & Intermol & S22 & 22 & 0.211 & -0.043 & 0.280 & 0.024 & 0.939 \\
\hline 47 & Intermol & S66 & 66 & 0.190 & -0.098 & 0.252 & 0.087 & 0.942 \\
\hline 48 & Conformer & SCONF & 17 & 0.035 & -0.001 & 0.052 & 0.005 & 0.840 \\
\hline 49 & Thermo & SIE4X4 & 16 & 5.678 & 5.678 & 6.712 & 0.102 & 1.057 \\
\hline 50 & Thermo & TAUT15 & 15 & 0.708 & -0.300 & 0.820 & 0.132 & 1.079 \\
\hline 51 & Conformer & UPU23 & 23 & 0.360 & 0.166 & 0.478 & 0.055 & 0.943 \\
\hline 52 & Thermo & W4-11 & 140 & 3.042 & -2.551 & 3.946 & 0.053 & 0.964 \\
\hline 53 & Intermol & WATER27 & 27 & 0.422 & 0.070 & 0.576 & 0.005 & 0.916 \\
\hline 54 & Barrier & WCPT18 & 18 & 1.338 & -1.338 & 1.670 & 0.026 & 1.002 \\
\hline 55 & Thermo & YBDE18 & 18 & 1.017 & 0.180 & 1.360 & 0.014 & 0.935 \\
\hline
\end{tabular}

$\underline{\text { Table S5: A breakdown by components of DSD-SCAN } 63-\mathrm{D} 3(\mathrm{BJ}) \text { taking } \mathrm{C}_{\mathrm{x}, \mathrm{HF}}=4^{-1 / 3} \approx 0.6300 \ldots}$

\begin{tabular}{|c|c|c|c|c|c|c|c|c|}
\hline $\begin{array}{c}\text { Serial } \\
\text { No. }\end{array}$ & Type & Set Name & $\begin{array}{l}\text { No. of } \\
\text { Points }\end{array}$ & MAD & MSD & RSMD & AWTMAD2 & 5MAD/4RMSD \\
\hline 1 & Conformer & ACONF & 15 & 0.080 & 0.080 & 0.097 & 0.025 & 1.025 \\
\hline 2 & Intermol & ADIM6 & 6 & 0.583 & -0.583 & 0.626 & 0.040 & 1.164 \\
\hline 3 & Intermol & AHB21 & 21 & 0.320 & -0.229 & 0.437 & 0.011 & 0.916 \\
\hline 4 & Thermo & AL2X6 & 6 & 0.374 & 0.374 & 0.430 & 0.002 & 1.086 \\
\hline 5 & Thermo & ALK8 & 8 & 1.130 & 0.908 & 1.562 & 0.006 & 0.904 \\
\hline 6 & Thermo & ALKBDE10 & 10 & 2.734 & -1.329 & 3.231 & 0.010 & 1.058 \\
\hline 7 & Conformer & AMINO20X4 & 80 & 0.121 & -0.025 & 0.154 & 0.151 & 0.982 \\
\hline 8 & Barrier & BH76RC & 30 & 1.452 & -0.221 & 1.803 & 0.077 & 1.007 \\
\hline 9 & Thermo & BH76 & 76 & 1.431 & -0.303 & 2.113 & 0.222 & 0.847 \\
\hline 10 & Barrier & BHDIV 10 & 10 & 1.182 & -0.829 & 1.333 & 0.010 & 1.109 \\
\hline 11 & Barrier & BHPERI & 26 & 1.290 & -1.185 & 1.442 & 0.061 & 1.119 \\
\hline 12 & Barrier & BHROT27 & 27 & 0.226 & 0.221 & 0.296 & 0.037 & 0.956 \\
\hline 13 & REACLarge & BSR36 & 36 & 1.012 & -1.012 & 1.057 & 0.085 & 1.196 \\
\hline 14 & Conformer & BUT14DIOL & 64 & 0.065 & -0.064 & 0.071 & 0.057 & 1.159 \\
\hline 15 & REACLarge & C60ISO & 9 & 6.459 & -6.364 & 8.630 & 0.022 & 0.936 \\
\hline 16 & Intermol & CARBHB12 & 12 & 0.331 & 0.315 & 0.484 & 0.025 & 0.855 \\
\hline 17 & REACLarge & CDIE20 & 20 & 0.408 & 0.408 & 0.470 & 0.076 & 1.087 \\
\hline 18 & Intermol & CHB6 & 6 & 0.965 & -0.965 & 1.154 & 0.008 & 1.046 \\
\hline 19 & REACLarge & DARC & 14 & 1.313 & -1.313 & 1.470 & 0.022 & 1.116 \\
\hline 20 & Thermo & DC13 & 13 & 2.377 & -0.190 & 3.356 & 0.021 & 0.885 \\
\hline 21 & Thermo & DIPCS10 & 10 & 3.367 & -3.367 & 4.154 & 0.002 & 1.013 \\
\hline 22 & Thermo & FH51 & 51 & 0.863 & -0.337 & 1.169 & 0.054 & 0.923 \\
\hline 23 & Thermo & G21EA & 25 & 2.780 & -2.258 & 3.249 & 0.078 & 1.070 \\
\hline 24 & Thermo & G21IP & 36 & 2.154 & -0.631 & 2.906 & 0.011 & 0.927 \\
\hline 25 & Thermo & G2RC & 25 & 2.073 & -0.081 & 2.608 & 0.038 & 0.994 \\
\hline 26 & Intermol & HAL59 & 59 & 0.279 & 0.144 & 0.405 & 0.136 & 0.862 \\
\hline 27 & Intermol & HEAVY28 & 28 & 0.097 & -0.035 & 0.122 & 0.083 & 0.995 \\
\hline 28 & Thermo & HEAVYSB11 & 11 & 0.957 & -0.517 & 1.237 & 0.007 & 0.968 \\
\hline 29 & Conformer & ICONF & 17 & 0.092 & 0.013 & 0.116 & 0.018 & 0.985 \\
\hline 30 & Conformer & IDISP & 6 & 0.662 & 0.170 & 0.815 & 0.011 & 1.014 \\
\hline 31 & Intermol & IL16 & 16 & 0.411 & 0.411 & 0.445 & 0.002 & 1.153 \\
\hline 32 & Barrier & INV24 & 24 & 0.652 & 0.291 & 1.115 & 0.019 & 0.730 \\
\hline 33 & REACLarge & ISO34 & 34 & 0.487 & -0.173 & 0.590 & 0.043 & 1.032 \\
\hline 34 & REACLarge & ISOL24 & 24 & 0.868 & 0.066 & 1.257 & 0.036 & 0.863 \\
\hline 35 & REACLarge & MB16-43 & 43 & 4.792 & 0.111 & 6.190 & 0.017 & 0.968 \\
\hline
\end{tabular}




\begin{tabular}{|l|l|l|r|r|r|r|r|c|}
\hline 36 & Conformer & MCONF & 51 & 0.258 & 0.211 & 0.293 & 0.100 & 1.101 \\
\hline 37 & Thermo & NBPRC & 6 & 0.735 & -0.594 & 0.860 & 0.007 & 1.068 \\
\hline 38 & Thermo & PA26 & 26 & 2.184 & 2.184 & 2.521 & 0.011 & 1.083 \\
\hline 39 & REACLarge & PAREL & 20 & 0.534 & 0.170 & 0.832 & 0.087 & 0.802 \\
\hline 40 & Conformer & PCONF21 & 18 & 0.214 & 0.116 & 0.277 & 0.090 & 0.964 \\
\hline 41 & Intermol & PNICO23 & 23 & 0.179 & 0.130 & 0.289 & 0.037 & 0.775 \\
\hline 42 & Barrier & PX13 & 13 & 1.900 & -1.900 & 2.006 & 0.028 & 1.184 \\
\hline 43 & Thermo & RC21 & 21 & 1.347 & -0.794 & 1.619 & 0.030 & 1.040 \\
\hline 44 & Intermol & RG18 & 18 & 0.073 & -0.050 & 0.106 & 0.086 & 0.862 \\
\hline 45 & REACLarge & RSE43 & 43 & 1.054 & 1.048 & 1.884 & 0.226 & 0.699 \\
\hline 46 & Intermol & S22 & 22 & 0.214 & -0.039 & 0.285 & 0.024 & 0.940 \\
\hline 47 & Intermol & S66 & 66 & 0.186 & -0.101 & 0.247 & 0.085 & 0.943 \\
\hline 48 & Conformer & SCONF & 17 & 0.037 & -0.010 & 0.044 & 0.005 & 1.050 \\
\hline 49 & Thermo & SIE4X4 & 16 & 4.831 & 4.831 & 5.769 & 0.087 & 1.047 \\
\hline 50 & Thermo & TAUT15 & 15 & 0.636 & -0.307 & 0.761 & 0.119 & 1.045 \\
\hline 51 & Conformer & UPU23 & 23 & 0.370 & 0.172 & 0.486 & 0.056 & 0.951 \\
\hline 52 & Thermo & W4-11 & 140 & 2.624 & -1.679 & 3.670 & 0.045 & 0.894 \\
\hline 53 & Intermol & WATER27 & 27 & 0.520 & -0.182 & 0.752 & 0.007 & 0.865 \\
\hline 54 & Barrier & WCPT18 & 18 & 1.095 & -1.079 & 1.412 & 0.021 & 0.969 \\
\hline 55 & Thermo & YBDE18 & 18 & 1.035 & 0.676 & 1.449 & 0.014 & 0.893 \\
\hline
\end{tabular}

Table S6: A breakdown by components of DSD-SCAN ${ }_{66}-\mathrm{D3}(\mathrm{BJ})$ taking $\mathrm{C}_{\mathrm{x}, \mathrm{HF}}=3.5^{-1 / 3} \approx 0.6586 \ldots$

\begin{tabular}{|c|c|c|c|c|c|c|c|c|}
\hline $\begin{array}{c}\text { Serial } \\
\text { No. }\end{array}$ & Type & Set Name & $\begin{array}{l}\text { No. of } \\
\text { Points } \\
\end{array}$ & MAD & MSD & RSMD & AWTMAD2 & 5MAD/4RMSD \\
\hline 1 & Conformer & ACONF & 15 & 0.083 & 0.083 & 0.101 & 0.026 & 1.024 \\
\hline 2 & Intermol & ADIM6 & 6 & 0.594 & -0.594 & 0.636 & 0.040 & 1.167 \\
\hline 3 & Intermol & AHB21 & 21 & 0.322 & -0.221 & 0.437 & 0.011 & 0.921 \\
\hline 4 & Thermo & AL2X6 & 6 & 0.191 & 0.174 & 0.256 & 0.001 & 0.933 \\
\hline 5 & Thermo & ALK8 & 8 & 0.990 & 0.643 & 1.288 & 0.005 & 0.961 \\
\hline 6 & Thermo & ALKBDE10 & 10 & 2.677 & -1.202 & 3.147 & 0.010 & 1.063 \\
\hline 7 & Conformer & AMINO20X4 & 80 & 0.122 & -0.027 & 0.155 & 0.152 & 0.982 \\
\hline 8 & Barrier & BH76RC & 30 & 1.372 & -0.231 & 1.688 & 0.073 & 1.016 \\
\hline 9 & Thermo & $\mathrm{BH76}$ & 76 & 1.418 & 0.020 & 2.214 & 0.220 & 0.801 \\
\hline 10 & Barrier & BHDIV10 & 10 & 1.093 & -0.711 & 1.231 & 0.009 & 1.110 \\
\hline 11 & Barrier & BHPERI & 26 & 1.350 & -1.243 & 1.495 & 0.064 & 1.129 \\
\hline 12 & Barrier & BHROT27 & 27 & 0.217 & 0.213 & 0.284 & 0.036 & 0.957 \\
\hline 13 & REACLarge & BSR36 & 36 & 0.871 & -0.871 & 0.899 & 0.073 & 1.211 \\
\hline 14 & Conformer & BUT14DIOL & 64 & 0.068 & -0.066 & 0.074 & 0.059 & 1.146 \\
\hline 15 & REACLarge & C60ISO & 9 & 6.759 & -6.670 & 9.024 & 0.024 & 0.936 \\
\hline 16 & Intermol & CARBHB12 & 12 & 0.321 & 0.302 & 0.468 & 0.024 & 0.857 \\
\hline 17 & REACLarge & CDIE20 & 20 & 0.408 & 0.408 & 0.464 & 0.076 & 1.098 \\
\hline 18 & Intermol & CHB6 & 6 & 0.962 & -0.962 & 1.150 & 0.008 & 1.046 \\
\hline 19 & REACLarge & DARC & 14 & 1.399 & -1.399 & 1.546 & 0.023 & 1.132 \\
\hline 20 & Thermo & DC13 & 13 & 2.488 & -0.119 & 3.384 & 0.022 & 0.919 \\
\hline 21 & Thermo & DIPCS10 & 10 & 3.359 & -3.359 & 4.186 & 0.002 & 1.003 \\
\hline 22 & Thermo & FH51 & 51 & 0.814 & -0.395 & 1.101 & 0.051 & 0.925 \\
\hline 23 & Thermo & G21EA & 25 & 2.762 & -2.211 & 3.251 & 0.078 & 1.062 \\
\hline 24 & Thermo & G21IP & 36 & 2.112 & -0.591 & 2.912 & 0.011 & 0.906 \\
\hline 25 & Thermo & G2RC & 25 & 1.909 & -0.181 & 2.409 & 0.035 & 0.991 \\
\hline 26 & Intermol & HAL59 & 59 & 0.266 & 0.123 & 0.381 & 0.130 & 0.874 \\
\hline 27 & Intermol & HEAVY28 & 28 & 0.089 & -0.032 & 0.112 & 0.076 & 0.992 \\
\hline 28 & Thermo & HEAVYSB11 & 11 & 0.949 & -0.468 & 1.197 & 0.007 & 0.992 \\
\hline 29 & Conformer & ICONF & 17 & 0.094 & 0.014 & 0.119 & 0.019 & 0.986 \\
\hline 30 & Conformer & IDISP & 6 & 0.755 & 0.078 & 0.949 & 0.012 & 0.994 \\
\hline 31 & Intermol & IL16 & 16 & 0.429 & 0.429 & 0.459 & 0.002 & 1.169 \\
\hline 32 & Barrier & INV24 & 24 & 0.672 & 0.287 & 1.133 & 0.019 & 0.741 \\
\hline 33 & REACLarge & ISO34 & 34 & 0.486 & -0.143 & 0.577 & 0.043 & 1.053 \\
\hline 34 & REACLarge & ISOL24 & 24 & 0.832 & 0.131 & 1.191 & 0.035 & 0.873 \\
\hline
\end{tabular}




\begin{tabular}{|l|l|l|r|r|r|r|r|c|}
\hline 35 & REACLarge & MB16-43 & 43 & 4.564 & 0.600 & 5.951 & 0.016 & 0.959 \\
\hline 36 & Conformer & MCONF & 51 & 0.268 & 0.220 & 0.301 & 0.104 & 1.112 \\
\hline 37 & Thermo & NBPRC & 6 & 0.665 & -0.517 & 0.777 & 0.006 & 1.069 \\
\hline 38 & Thermo & PA26 & 26 & 1.996 & 1.996 & 2.322 & 0.010 & 1.074 \\
\hline 39 & REACLarge & PAREL & 20 & 0.519 & 0.173 & 0.795 & 0.085 & 0.816 \\
\hline 40 & Conformer & PCONF21 & 18 & 0.212 & 0.121 & 0.275 & 0.089 & 0.967 \\
\hline 41 & Intermol & PNICO23 & 23 & 0.151 & 0.096 & 0.246 & 0.031 & 0.764 \\
\hline 42 & Barrier & PX13 & 13 & 1.788 & -1.788 & 1.900 & 0.026 & 1.176 \\
\hline 43 & Thermo & RC21 & 21 & 1.561 & -1.080 & 1.889 & 0.035 & 1.033 \\
\hline 44 & Intermol & RG18 & 18 & 0.077 & -0.056 & 0.109 & 0.091 & 0.882 \\
\hline 45 & REACLarge & RSE43 & 43 & 1.154 & 1.151 & 2.086 & 0.248 & 0.692 \\
\hline 46 & Intermol & S22 & 22 & 0.217 & -0.037 & 0.289 & 0.025 & 0.941 \\
\hline 47 & Intermol & S66 & 66 & 0.189 & -0.107 & 0.250 & 0.086 & 0.943 \\
\hline 48 & Conformer & SCONF & 17 & 0.037 & -0.008 & 0.043 & 0.005 & 1.065 \\
\hline 49 & Thermo & SIE4X4 & 16 & 4.273 & 4.273 & 5.122 & 0.077 & 1.043 \\
\hline 50 & Thermo & TAUT15 & 15 & 0.586 & -0.273 & 0.696 & 0.109 & 1.053 \\
\hline 51 & Conformer & UPU23 & 23 & 0.372 & 0.168 & 0.488 & 0.057 & 0.953 \\
\hline 52 & Thermo & W4-11 & 140 & 2.489 & -1.550 & 3.554 & 0.043 & 0.876 \\
\hline 53 & Intermol & WATER27 & 27 & 0.639 & -0.362 & 0.954 & 0.008 & 0.837 \\
\hline 54 & Barrier & WCPT18 & 18 & 1.034 & -1.003 & 1.323 & 0.020 & 0.978 \\
\hline 55 & Thermo & YBDE18 & 18 & 1.162 & 0.882 & 1.599 & 0.016 & 0.908 \\
\hline
\end{tabular}

$\underline{\text { Table S7: A breakdown by components of DSD-SCAN } 69-\mathrm{D} 3(\mathrm{BJ}) \text { taking } \mathrm{C}_{\mathrm{x}, \mathrm{HF}}=3^{-1 / 3} \approx 0.69336 \ldots}$

\begin{tabular}{|c|c|c|c|c|c|c|c|c|}
\hline $\begin{array}{c}\text { Serial } \\
\text { No. }\end{array}$ & Type & Set Name & $\begin{array}{l}\text { No. of } \\
\text { Points }\end{array}$ & MAD & MSD & RSMD & $\triangle \mathrm{WTMAD2}$ & 5MAD/4RMSD \\
\hline 1 & Conformer & ACONF & 15 & 0.073 & 0.073 & 0.093 & 0.023 & 0.984 \\
\hline 2 & Intermol & ADIM6 & 6 & 0.590 & -0.590 & 0.632 & 0.040 & 1.167 \\
\hline 3 & Intermol & AHB21 & 21 & 0.322 & -0.223 & 0.436 & 0.011 & 0.922 \\
\hline 4 & Thermo & AL2X6 & 6 & 0.244 & 0.244 & 0.328 & 0.002 & 0.929 \\
\hline 5 & Thermo & ALK8 & 8 & 0.968 & 0.656 & 1.208 & 0.005 & 1.002 \\
\hline 6 & Thermo & ALKBDE10 & 10 & 2.586 & -1.163 & 3.015 & 0.010 & 1.072 \\
\hline 7 & Conformer & AMINO20X4 & 80 & 0.120 & -0.026 & 0.154 & 0.150 & 0.974 \\
\hline 8 & Barrier & BH76RC & 30 & 1.333 & -0.241 & 1.612 & 0.071 & 1.033 \\
\hline 9 & Thermo & BH76 & 76 & 1.462 & 0.361 & 2.397 & 0.226 & 0.762 \\
\hline 10 & Barrier & BHDIV10 & 10 & 0.974 & -0.584 & 1.110 & 0.008 & 1.098 \\
\hline 11 & Barrier & BHPERI & 26 & 1.381 & -1.266 & 1.547 & 0.065 & 1.115 \\
\hline 12 & Barrier & BHROT27 & 27 & 0.209 & 0.205 & 0.271 & 0.034 & 0.965 \\
\hline 13 & REACLarge & BSR36 & 36 & 0.699 & -0.699 & 0.721 & 0.059 & 1.212 \\
\hline 14 & Conformer & BUT14DIOL & 64 & 0.070 & -0.069 & 0.076 & 0.061 & 1.150 \\
\hline 15 & REACLarge & C60ISO & 9 & 6.703 & -6.600 & 8.996 & 0.023 & 0.931 \\
\hline 16 & Intermol & CARBHB12 & 12 & 0.313 & 0.294 & 0.452 & 0.024 & 0.866 \\
\hline 17 & REACLarge & CDIE20 & 20 & 0.373 & 0.373 & 0.428 & 0.070 & 1.090 \\
\hline 18 & Intermol & CHB6 & 6 & 1.010 & -1.010 & 1.200 & 0.009 & 1.052 \\
\hline 19 & REACLarge & DARC & 14 & 1.705 & -1.705 & 1.816 & 0.028 & 1.173 \\
\hline 20 & Thermo & DC13 & 13 & 2.717 & -0.267 & 3.514 & 0.024 & 0.967 \\
\hline 21 & Thermo & DIPCS10 & 10 & 3.373 & -3.358 & 4.214 & 0.002 & 1.001 \\
\hline 22 & Thermo & FH51 & 51 & 0.816 & -0.480 & 1.101 & 0.051 & 0.927 \\
\hline 23 & Thermo & G21EA & 25 & 2.805 & -2.216 & 3.324 & 0.079 & 1.055 \\
\hline 24 & Thermo & G21IP & 36 & 2.079 & -0.561 & 2.948 & 0.011 & 0.882 \\
\hline 25 & Thermo & G2RC & 25 & 1.800 & -0.278 & 2.278 & 0.033 & 0.988 \\
\hline 26 & Intermol & HAL59 & 59 & 0.258 & 0.110 & 0.369 & 0.126 & 0.875 \\
\hline 27 & Intermol & HEAVY28 & 28 & 0.090 & -0.036 & 0.112 & 0.077 & 1.004 \\
\hline 28 & Thermo & HEAVYSB11 & 11 & 0.954 & -0.248 & 1.142 & 0.007 & 1.045 \\
\hline 29 & Conformer & ICONF & 17 & 0.093 & 0.017 & 0.121 & 0.018 & 0.965 \\
\hline 30 & Conformer & IDISP & 6 & 0.798 & -0.051 & 1.147 & 0.013 & 0.870 \\
\hline 31 & Intermol & IL16 & 16 & 0.412 & 0.412 & 0.442 & 0.002 & 1.164 \\
\hline
\end{tabular}




\begin{tabular}{|l|l|l|r|r|r|r|r|c|}
\hline 32 & Barrier & INV24 & 23 & 0.705 & 0.351 & 1.190 & 0.020 & 0.740 \\
\hline 33 & REACLarge & ISO34 & 34 & 0.471 & -0.110 & 0.565 & 0.042 & 1.043 \\
\hline 34 & REACLarge & ISOL24 & 24 & 0.837 & 0.281 & 1.131 & 0.035 & 0.926 \\
\hline 35 & REACLarge & MB16-43 & 43 & 4.825 & 1.929 & 6.351 & 0.017 & 0.950 \\
\hline 36 & Conformer & MCONF & 51 & 0.274 & 0.228 & 0.307 & 0.107 & 1.116 \\
\hline 37 & Thermo & NBPRC & 6 & 0.668 & -0.520 & 0.772 & 0.006 & 1.082 \\
\hline 38 & Thermo & PA26 & 26 & 1.928 & 1.928 & 2.236 & 0.010 & 1.078 \\
\hline 39 & REACLarge & PAREL & 20 & 0.493 & 0.169 & 0.743 & 0.081 & 0.829 \\
\hline 40 & Conformer & PCONF21 & 18 & 0.214 & 0.120 & 0.277 & 0.090 & 0.968 \\
\hline 41 & Intermol & PNICO23 & 23 & 0.150 & 0.086 & 0.239 & 0.031 & 0.784 \\
\hline 42 & Barrier & PX13 & 13 & 1.606 & -1.606 & 1.724 & 0.024 & 1.164 \\
\hline 43 & Thermo & RC21 & 21 & 1.793 & -1.303 & 2.145 & 0.040 & 1.045 \\
\hline 44 & Intermol & RG18 & 18 & 0.081 & -0.061 & 0.116 & 0.095 & 0.872 \\
\hline 45 & REACLarge & RSE43 & 43 & 1.284 & 1.283 & 2.314 & 0.275 & 0.694 \\
\hline 46 & Intermol & S22 & 22 & 0.217 & -0.028 & 0.285 & 0.025 & 0.953 \\
\hline 47 & Intermol & S66 & 66 & 0.186 & -0.104 & 0.247 & 0.085 & 0.941 \\
\hline 48 & Conformer & SCONF & 17 & 0.029 & 0.001 & 0.036 & 0.004 & 1.007 \\
\hline 49 & Thermo & SIE4X4 & 16 & 3.687 & 3.687 & 4.466 & 0.066 & 1.032 \\
\hline 50 & Thermo & TAUT15 & 15 & 0.539 & -0.262 & 0.651 & 0.101 & 1.034 \\
\hline 51 & Conformer & UPU23 & 23 & 0.378 & 0.177 & 0.491 & 0.058 & 0.962 \\
\hline 52 & Thermo & W4-11 & 140 & 2.495 & -1.397 & 3.628 & 0.043 & 0.860 \\
\hline 53 & Intermol & WATER27 & 27 & 0.652 & -0.394 & 0.989 & 0.008 & 0.824 \\
\hline 54 & Barrier & WCPT18 & 18 & 0.926 & -0.853 & 1.197 & 0.018 & 0.967 \\
\hline 55 & Thermo & YBDE18 & 18 & 1.350 & 1.234 & 1.826 & 0.019 & 0.924 \\
\hline
\end{tabular}

Table S8: A breakdown by components of DSD-SCAN ${ }_{74}-\mathrm{D3}(\mathrm{BJ})$ taking $\mathrm{C}_{\mathrm{x}, \mathrm{HF}}=2.5^{-1 / 3} \approx 0.7368 \ldots$

\begin{tabular}{|c|c|c|c|c|c|c|c|c|}
\hline $\begin{array}{c}\text { Serial } \\
\text { No. }\end{array}$ & Type & Set Name & $\begin{array}{l}\text { No. of } \\
\text { Points }\end{array}$ & MAD & MSD & RSMD & AWTMAD2 & 5MAD/4RMSD \\
\hline 1 & Conformer & $\mathrm{ACONF}$ & 15 & 0.070 & 0.070 & 0.092 & 0.022 & 0.950 \\
\hline 2 & Intermol & ADIM6 & 6 & 0.593 & -0.593 & 0.633 & 0.040 & 1.170 \\
\hline 3 & Intermol & AHB21 & 21 & 0.336 & -0.242 & 0.456 & 0.012 & 0.921 \\
\hline 4 & Thermo & AL2X6 & 6 & 0.176 & 0.130 & 0.254 & 0.001 & 0.864 \\
\hline 5 & Thermo & ALK8 & 8 & 0.827 & 0.455 & 0.956 & 0.004 & 1.082 \\
\hline 6 & Thermo & ALKBDE10 & 10 & 2.491 & -1.108 & 2.866 & 0.009 & 1.087 \\
\hline 7 & Conformer & AMINO20X4 & 80 & 0.120 & -0.025 & 0.155 & 0.150 & 0.972 \\
\hline 8 & Barrier & BH76RC & 30 & 1.256 & -0.277 & 1.530 & 0.067 & 1.026 \\
\hline 9 & Thermo & BH76 & 76 & 1.648 & 0.889 & 2.765 & 0.255 & 0.745 \\
\hline 10 & Barrier & BHDIV10 & 10 & 0.834 & -0.382 & 0.956 & 0.007 & 1.090 \\
\hline 11 & Barrier & BHPERI & 26 & 1.431 & -1.305 & 1.620 & 0.068 & 1.104 \\
\hline 12 & Barrier & BHROT27 & 27 & 0.202 & 0.199 & 0.262 & 0.033 & 0.965 \\
\hline 13 & REACLarge & BSR36 & 36 & 0.490 & -0.478 & 0.529 & 0.041 & 1.158 \\
\hline 14 & Conformer & BUT14DIOL & 64 & 0.071 & -0.070 & 0.078 & 0.062 & 1.138 \\
\hline 15 & REACLarge & C60ISO & 9 & 6.736 & -6.615 & 9.106 & 0.023 & 0.925 \\
\hline 16 & Intermol & CARBHB12 & 12 & 0.307 & 0.288 & 0.438 & 0.023 & 0.876 \\
\hline 17 & REACLarge & CDIE20 & 20 & 0.353 & 0.353 & 0.408 & 0.066 & 1.082 \\
\hline 18 & Intermol & CHB6 & 6 & 1.055 & -1.055 & 1.244 & 0.009 & 1.060 \\
\hline 19 & REACLarge & DARC & 14 & 2.081 & -2.081 & 2.175 & 0.034 & 1.196 \\
\hline 20 & Thermo & $\mathrm{DC} 13$ & 13 & 3.056 & -0.357 & 3.781 & 0.027 & 1.010 \\
\hline 21 & Thermo & DIPCS10 & 10 & 3.371 & -3.249 & 4.228 & 0.002 & 0.997 \\
\hline 22 & Thermo & FH51 & 51 & 0.865 & -0.632 & 1.181 & 0.054 & 0.916 \\
\hline 23 & Thermo & G21EA & 25 & 2.814 & -2.167 & 3.407 & 0.079 & 1.032 \\
\hline 24 & Thermo & G21IP & 36 & 2.010 & -0.463 & 3.014 & 0.011 & 0.834 \\
\hline 25 & Thermo & G2RC & 25 & 1.627 & -0.519 & 2.104 & 0.030 & 0.967 \\
\hline
\end{tabular}




\begin{tabular}{|c|c|c|c|c|c|c|c|c|}
\hline 26 & Intermol & HAL59 & 59 & 0.243 & 0.086 & 0.348 & 0.118 & 0.873 \\
\hline 27 & Intermol & HEAVY28 & 28 & 0.083 & -0.031 & 0.104 & 0.071 & 0.998 \\
\hline 28 & Thermo & HEAVYSB11 & 11 & 0.966 & -0.079 & 1.117 & 0.007 & 1.081 \\
\hline 29 & Conformer & ICONF & 17 & 0.095 & 0.022 & 0.126 & 0.019 & 0.941 \\
\hline 30 & Conformer & IDISP & 6 & 0.963 & -0.237 & 1.491 & 0.015 & 0.807 \\
\hline 31 & Intermol & IL16 & 16 & 0.404 & 0.404 & 0.433 & 0.002 & 1.165 \\
\hline 32 & Barrier & INV24 & 24 & 0.756 & 0.400 & 1.256 & 0.022 & 0.753 \\
\hline 33 & REACLarge & ISO34 & 34 & 0.468 & -0.058 & 0.598 & 0.042 & 0.979 \\
\hline 34 & REACLarge & ISOL24 & 24 & 0.889 & 0.462 & 1.183 & 0.037 & 0.939 \\
\hline 35 & REACLarge & MB16-43 & 43 & 5.375 & 3.496 & 7.249 & 0.019 & 0.927 \\
\hline 36 & Conformer & MCONF & 51 & 0.288 & 0.242 & 0.320 & 0.112 & 1.123 \\
\hline 37 & Thermo & NBPRC & 6 & 0.615 & -0.482 & 0.717 & 0.006 & 1.073 \\
\hline 38 & Thermo & PA26 & 26 & 1.714 & 1.714 & 2.015 & 0.009 & 1.063 \\
\hline 39 & REACLarge & PAREL & 20 & 0.466 & 0.180 & 0.679 & 0.076 & 0.858 \\
\hline 40 & Conformer & PCONF21 & 18 & 0.215 & 0.126 & 0.276 & 0.090 & 0.972 \\
\hline 41 & Intermol & PNICO23 & 23 & 0.133 & 0.057 & 0.204 & 0.027 & 0.814 \\
\hline 42 & Barrier & PX13 & 13 & 1.393 & -1.393 & 1.529 & 0.021 & 1.139 \\
\hline 43 & Thermo & $\mathrm{RC} 21$ & 21 & 2.261 & -1.686 & 2.607 & 0.050 & 1.084 \\
\hline 44 & Intermol & RG18 & 18 & 0.086 & -0.069 & 0.123 & 0.102 & 0.879 \\
\hline 45 & REACLarge & RSE43 & 43 & 1.462 & 1.462 & 2.648 & 0.314 & 0.690 \\
\hline 46 & Intermol & S22 & 22 & 0.221 & -0.012 & 0.283 & 0.025 & 0.976 \\
\hline 47 & Intermol & S66 & 66 & 0.184 & -0.100 & 0.245 & 0.084 & 0.940 \\
\hline 48 & Conformer & SCONF & 17 & 0.028 & 0.009 & 0.040 & 0.004 & 0.866 \\
\hline 49 & Thermo & SIE4X4 & 16 & 2.784 & 2.775 & 3.451 & 0.050 & 1.008 \\
\hline 50 & Thermo & TAUT15 & 15 & 0.465 & -0.214 & 0.558 & 0.087 & 1.043 \\
\hline 51 & Conformer & UPU23 & 23 & 0.385 & 0.181 & 0.497 & 0.059 & 0.970 \\
\hline 52 & Thermo & W4-11 & 140 & 2.514 & -1.248 & 3.748 & 0.044 & 0.838 \\
\hline 53 & Intermol & WATER 27 & 27 & 0.727 & -0.490 & 1.126 & 0.009 & 0.808 \\
\hline 54 & Barrier & WCPT18 & 18 & 0.820 & -0.668 & 1.047 & 0.016 & 0.980 \\
\hline 55 & Thermo & YBDE18 & 18 & 1.690 & 1.653 & 2.188 & 0.023 & 0.966 \\
\hline
\end{tabular}

Table S9: A breakdown by components of DSD-SCAN $79-D 3(B J)$ [DSD-SCAN0-2-D3(BJ)] taking $\mathrm{C}_{\mathrm{x}, \mathrm{HF}}=\mathbf{2}^{-1 / 3} \approx 0.7937 \ldots$

\begin{tabular}{|l|l|l|r|r|r|r|r|c|}
\hline $\begin{array}{l}\text { Seria } \\
\text { I No. }\end{array}$ & \multicolumn{1}{|c|}{ Type } & Set Name & $\begin{array}{c}\text { No. of } \\
\text { Points }\end{array}$ & MAD & MSD & RSMD & $\Delta$ WTMAD2 & 5MAD/4RMSD \\
\hline 1 & Conformer & ACONF & 15 & 0.071 & 0.071 & 0.095 & 0.022 & 0.934 \\
\hline 2 & Intermol & ADIM6 & 6 & 0.612 & -0.612 & 0.652 & 0.041 & 1.172 \\
\hline 3 & Intermol & AHB21 & 21 & 0.359 & -0.268 & 0.493 & 0.013 & 0.909 \\
\hline 4 & Thermo & AL2X6 & 6 & 0.222 & -0.128 & 0.236 & 0.001 & 1.179 \\
\hline 5 & Thermo & ALK8 & 8 & 0.620 & 0.098 & 0.738 & 0.003 & 1.050 \\
\hline 6 & Thermo & ALKBDE10 & 10 & 2.339 & -1.038 & 2.708 & 0.009 & 1.080 \\
\hline 7 & Conformer & AMINO20X4 & 80 & 0.124 & -0.025 & 0.158 & 0.154 & 0.978 \\
\hline 8 & Barrier & BH76RC & 30 & 1.186 & -0.342 & 1.525 & 0.063 & 0.972 \\
\hline 9 & Thermo & BH76 & 76 & 1.996 & 1.550 & 3.338 & 0.309 & 0.748 \\
\hline 10 & Barrier & BHDIV10 & 10 & 0.708 & -0.101 & 0.800 & 0.006 & 1.107 \\
\hline 11 & Barrier & BHPERI & 26 & 1.424 & -1.253 & 1.628 & 0.067 & 1.093 \\
\hline 12 & Barrier & BHROT27 & 27 & 0.193 & 0.191 & 0.255 & 0.032 & 0.947 \\
\hline 13 & REACLarge & BSR36 & 36 & 0.438 & -0.298 & 0.462 & 0.037 & 1.184 \\
\hline 14 & Conformer & BUT14DIOL & 64 & 0.074 & -0.071 & 0.083 & 0.064 & 1.109 \\
\hline 15 & REACLarge & C60ISO & 9 & 6.691 & -6.547 & 9.132 & 0.023 & 0.916 \\
\hline 16 & Intermol & CARBHB12 & 12 & 0.297 & 0.278 & 0.419 & 0.022 & 0.888 \\
\hline 17 & REACLarge & CDIE20 & 20 & 0.337 & 0.336 & 0.398 & 0.063 & 1.058 \\
\hline 18 & Intermol & CHB6 & 6 & 1.106 & -1.106 & 1.296 & 0.009 & 1.067 \\
\hline
\end{tabular}




\begin{tabular}{|c|c|c|c|c|c|c|c|c|}
\hline 19 & REACLarge & DARC & 14 & 2.497 & -2.497 & 2.582 & 0.041 & 1.209 \\
\hline 20 & Thermo & $\mathrm{DC} 13$ & 13 & 3.461 & -0.468 & 4.205 & 0.031 & 1.029 \\
\hline 21 & Thermo & DIPCS10 & 10 & 3.230 & -2.923 & 4.156 & 0.002 & 0.971 \\
\hline 22 & Thermo & FH51 & 51 & 1.024 & -0.824 & 1.394 & 0.064 & 0.919 \\
\hline 23 & Thermo & G21EA & 25 & 2.808 & -2.030 & 3.509 & 0.079 & 1.001 \\
\hline 24 & Thermo & G21IP & 36 & 1.974 & -0.248 & 3.138 & 0.011 & 0.786 \\
\hline 25 & Thermo & G2RC & 25 & 1.569 & -0.860 & 2.014 & 0.029 & 0.974 \\
\hline 26 & Intermol & HAL59 & 59 & 0.237 & 0.048 & 0.327 & 0.116 & 0.907 \\
\hline 27 & Intermol & HEAVY28 & 28 & 0.072 & -0.023 & 0.091 & 0.062 & 0.987 \\
\hline 28 & Thermo & HEAVYSB11 & 11 & 0.977 & 0.063 & 1.121 & 0.007 & 1.089 \\
\hline 29 & Conformer & ICONF & 17 & 0.102 & 0.024 & 0.137 & 0.020 & 0.934 \\
\hline 30 & Conformer & IDISP & 6 & 1.207 & -0.459 & 1.913 & 0.019 & 0.788 \\
\hline 31 & Intermol & IL16 & 16 & 0.413 & 0.413 & 0.443 & 0.002 & 1.165 \\
\hline 32 & Barrier & INV24 & 24 & 0.817 & 0.438 & 1.336 & 0.023 & 0.764 \\
\hline 33 & REACLarge & ISO34 & 34 & 0.515 & -0.005 & 0.696 & 0.046 & 0.925 \\
\hline 34 & REACLarge & ISOL24 & 24 & 0.996 & 0.646 & 1.396 & 0.041 & 0.892 \\
\hline 35 & REACLarge & MB16-43 & 43 & 6.629 & 4.879 & 8.662 & 0.023 & 0.957 \\
\hline 36 & Conformer & MCONF & 51 & 0.297 & 0.253 & 0.329 & 0.116 & 1.127 \\
\hline 37 & Thermo & NBPRC & 6 & 0.512 & -0.413 & 0.623 & 0.005 & 1.027 \\
\hline 38 & Thermo & PA26 & 26 & 1.454 & 1.443 & 1.762 & 0.008 & 1.032 \\
\hline 39 & REACLarge & PAREL & 20 & 0.446 & 0.199 & 0.614 & 0.073 & 0.909 \\
\hline 40 & Conformer & PCONF21 & 18 & 0.214 & 0.137 & 0.268 & 0.090 & 0.995 \\
\hline 41 & Intermol & $\mathrm{PNICO} 23$ & 23 & 0.106 & 0.009 & 0.150 & 0.022 & 0.884 \\
\hline 42 & Barrier & PX13 & 13 & 1.176 & -1.140 & 1.315 & 0.017 & 1.118 \\
\hline 43 & Thermo & $\mathrm{RC} 21$ & 21 & 2.876 & -2.198 & 3.256 & 0.064 & 1.104 \\
\hline 44 & Intermol & RG18 & 18 & 0.093 & -0.078 & 0.131 & 0.110 & 0.893 \\
\hline 45 & REACLarge & RSE43 & 43 & 1.678 & 1.678 & 3.068 & 0.360 & 0.684 \\
\hline 46 & Intermol & $\mathrm{S} 22$ & 22 & 0.226 & -0.004 & 0.284 & 0.026 & 0.992 \\
\hline 47 & Intermol & S66 & 66 & 0.187 & -0.107 & 0.251 & 0.086 & 0.932 \\
\hline 48 & Conformer & SCONF & 17 & 0.038 & 0.009 & 0.059 & 0.005 & 0.810 \\
\hline 49 & Thermo & SIE4X4 & 16 & 1.807 & 1.662 & 2.285 & 0.033 & 0.988 \\
\hline 50 & Thermo & TAUT15 & 15 & 0.385 & -0.156 & 0.450 & 0.072 & 1.071 \\
\hline 51 & Conformer & UPU23 & 23 & 0.394 & 0.190 & 0.506 & 0.060 & 0.973 \\
\hline 52 & Thermo & W4-11 & 140 & 2.668 & -0.883 & 4.024 & 0.046 & 0.829 \\
\hline 53 & Intermol & WATER27 & 27 & 0.879 & -0.657 & 1.378 & 0.011 & 0.797 \\
\hline 54 & Barrier & WCPT18 & 18 & 0.709 & -0.413 & 0.875 & 0.014 & 1.014 \\
\hline 55 & Thermo & YBDE18 & 18 & 2.125 & 2.125 & 2.626 & 0.029 & 1.011 \\
\hline
\end{tabular}


Table S10: WTMAD2 contribution for each of five major subcategories in cases of DSD-SCAN

\begin{tabular}{|c|c|c|c|c|c|c|c|c|}
\hline \multirow{2}{*}{ Type } & \multicolumn{7}{|c|}{$\mathbf{C}_{\mathbf{x}, \mathbf{H F}=\mathbf{n}} \mathbf{- 1 / 3}$} \\
\cline { 2 - 11 } & $\mathbf{n = 8}$ & $\mathbf{n = 6}$ & $\mathbf{n = 5}$ & $\mathbf{n = 4}$ & $\mathbf{n = 3 . 5}$ & $\mathbf{n = 3 . 0}$ & $\mathbf{n}=\mathbf{2 . 5}$ & $\mathbf{n = 2}$ \\
\hline Intermol & 0.622 & 0.584 & 0.570 & 0.543 & 0.533 & 0.533 & 0.523 & 0.520 \\
\hline Conformer & 0.513 & 0.503 & 0.499 & 0.514 & 0.522 & 0.523 & 0.532 & 0.550 \\
\hline Barrier & 0.534 & 0.479 & 0.436 & 0.252 & 0.247 & 0.240 & 0.233 & 0.222 \\
\hline Thermo & 0.821 & 0.717 & 0.671 & 0.764 & 0.739 & 0.735 & 0.749 & 0.801 \\
\hline REAClarge & 0.613 & 0.610 & 0.608 & 0.617 & 0.622 & 0.629 & 0.652 & 0.707 \\
\hline Total WTMAD2 & $\mathbf{3 . 1 0 4}$ & $\mathbf{2 . 8 9 3}$ & $\mathbf{2 . 7 8 5}$ & $\mathbf{2 . 6 9 0}$ & $\mathbf{2 . 6 6 2}$ & $\mathbf{2 . 6 6 0}$ & $\mathbf{2 . 6 8 8}$ & $\mathbf{2 . 8 0 0}$ \\
\hline
\end{tabular}

Table S11: Breakdown by Components for WTMAD2 comparison among above three functionals

\begin{tabular}{|c|c|c|c|c|c|c|c|}
\hline \multirow[t]{2}{*}{ Type } & \multirow[t]{2}{*}{ Set Name } & \multirow{2}{*}{$\begin{array}{l}\text { No. of } \\
\text { Points }\end{array}$} & \multicolumn{5}{|c|}{$\Delta$ WTMAD2(kcal/mol) } \\
\hline & & & B3LYP-D3(BJ) & PBE0-D3(BJ) & $\omega B 97 X-V$ & $\omega B 97 M-V$ & $\omega \mathrm{B97M}(2)$ \\
\hline Conformer & ACONF & 15 & 0.024 & 0.017 & 0.008 & 0.021 & 0.034 \\
\hline Intermol & ADIM6 & 6 & 0.012 & 0.004 & 0.009 & 0.007 & 0.029 \\
\hline Intermol & AHB21 & 21 & 0.017 & 0.048 & 0.012 & 0.007 & 0.009 \\
\hline Thermo & AL2X6 & 6 & 0.016 & 0.009 & 0.008 & 0.008 & 0.009 \\
\hline Thermo & ALK8 & 8 & 0.012 & 0.023 & 0.004 & 0.012 & 0.010 \\
\hline Thermo & ALKBDE10 & 10 & 0.016 & 0.021 & 0.015 & 0.014 & 0.013 \\
\hline Conformer & AMINO20X4 & 80 & 0.258 & 0.345 & 0.243 & 0.231 & 0.099 \\
\hline Barrier & $\mathrm{BH} 76 \mathrm{RC}$ & 30 & 0.117 & 0.131 & 0.096 & 0.061 & 0.055 \\
\hline Thermo & BH76 & 76 & 0.849 & 0.704 & 0.286 & 0.258 & 0.144 \\
\hline Barrier & BHDIV10 & 10 & 0.027 & 0.040 & 0.007 & 0.011 & 0.006 \\
\hline Barrier & BHPERI & 26 & 0.057 & 0.153 & 0.099 & 0.053 & 0.024 \\
\hline Barrier & BHROT27 & 27 & 0.069 & 0.095 & 0.049 & 0.038 & 0.027 \\
\hline REACLarge & BSR36 & 36 & 0.301 & 0.294 & 0.184 & 0.033 & 0.054 \\
\hline Conformer & BUT14DIOL & 64 & 0.299 & 0.210 & 0.052 & 0.056 & 0.040 \\
\hline REACLarge & C60ISO & 9 & 0.008 & 0.009 & 0.047 & 0.041 & 0.008 \\
\hline Intermol & CARBHB12 & 12 & 0.072 & 0.110 & 0.027 & 0.014 & 0.016 \\
\hline REACLarge & CDIE20 & 20 & 0.187 & 0.231 & 0.115 & 0.110 & 0.041 \\
\hline Intermol & CHB6 & 6 & 0.012 & 0.012 & 0.008 & 0.008 & 0.007 \\
\hline REACLarge & DARC & 14 & 0.127 & 0.061 & 0.070 & 0.012 & 0.025 \\
\hline Thermo & $\mathrm{DC} 13$ & 13 & 0.094 & 0.072 & 0.059 & 0.049 & 0.015 \\
\hline Thermo & DIPCS10 & 10 & 0.003 & 0.002 & 0.002 & 0.003 & 0.001 \\
\hline Thermo & FH51 & 51 & 0.159 & 0.173 & 0.144 & 0.067 & 0.041 \\
\hline Thermo & G21EA & 25 & 0.063 & 0.083 & 0.073 & 0.064 & 0.042 \\
\hline Thermo & G21IP & 36 & 0.020 & 0.020 & 0.016 & 0.015 & 0.008 \\
\hline Thermo & G2RC & 25 & 0.051 & 0.125 & 0.073 & 0.038 & 0.025 \\
\hline Intermol & HAL59 & 59 & 0.301 & 0.299 & 0.150 & 0.140 & 0.131 \\
\hline Intermol & HEAVY28 & 28 & 0.312 & 0.261 & 0.154 & 0.156 & 0.087 \\
\hline Thermo & HEAVYSB11 & 11 & 0.022 & 0.010 & 0.010 & 0.019 & 0.006 \\
\hline Conformer & ICONF & 17 & 0.055 & 0.056 & 0.050 & 0.028 & 0.024 \\
\hline Conformer & IDISP & 6 & 0.057 & 0.025 & 0.041 & 0.026 & 0.021 \\
\hline Intermol & IL16 & 16 & 0.004 & 0.002 & 0.005 & 0.004 & 0.001 \\
\hline Barrier & INV24 & 24 & 0.029 & 0.033 & 0.048 & 0.038 & 0.027 \\
\hline REACLarge & ISO34 & 34 & 0.155 & 0.126 & 0.103 & 0.055 & 0.034 \\
\hline REACLarge & ISOL24 & 24 & 0.237 & 0.088 & 0.123 & 0.068 & 0.043 \\
\hline REACLarge & MB16-43 & 43 & 0.084 & 0.055 & 0.115 & 0.052 & 0.047 \\
\hline Conformer & MCONF & 51 & 0.087 & 0.106 & 0.090 & 0.152 & 0.125 \\
\hline Thermo & NBPRC & 6 & 0.007 & 0.023 & 0.010 & 0.005 & 0.005 \\
\hline Thermo & PA26 & 26 & 0.015 & 0.015 & 0.014 & 0.007 & 0.006 \\
\hline REACLarge & PAREL & 20 & 0.191 & 0.197 & 0.104 & 0.095 & 0.070 \\
\hline Conformer & PCONF21 & 18 & 0.228 & 0.382 & 0.128 & 0.267 & 0.138 \\
\hline
\end{tabular}




\begin{tabular}{|l|l|l|l|l|l|l|l|}
\hline Intermol & PNICO23 & 23 & 0.111 & 0.196 & 0.037 & 0.053 & 0.043 \\
\hline Barrier & PX13 & 13 & 0.065 & 0.097 & 0.038 & 0.029 & 0.008 \\
\hline Thermo & RC21 & 21 & 0.059 & 0.126 & 0.079 & 0.039 & 0.024 \\
\hline Intermol & RG18 & 18 & 0.157 & 0.106 & 0.082 & 0.072 & 0.080 \\
\hline REACLarge & RSE43 & 43 & 0.372 & 0.309 & 0.207 & 0.173 & 0.095 \\
\hline Intermol & S22 & 22 & 0.042 & 0.059 & 0.026 & 0.028 & 0.016 \\
\hline Intermol & S66 & 66 & 0.152 & 0.171 & 0.056 & 0.070 & 0.067 \\
\hline Conformer & SCONF & 17 & 0.043 & 0.038 & 0.021 & 0.030 & 0.031 \\
\hline Thermo & SIE4X4 & 16 & 0.324 & 0.259 & 0.206 & 0.193 & 0.094 \\
\hline Thermo & TAUT15 & 15 & 0.220 & 0.211 & 0.134 & 0.058 & 0.050 \\
\hline Conformer & UPU23 & 23 & 0.097 & 0.087 & 0.097 & 0.084 & 0.066 \\
\hline Thermo & W4-11 & 140 & 0.056 & 0.061 & 0.048 & 0.036 & 0.024 \\
\hline Intermol & WATER27 & 27 & 0.048 & 0.067 & 0.012 & 0.005 & 0.008 \\
\hline Barrier & WCPT18 & 18 & 0.046 & 0.085 & 0.034 & 0.028 & 0.021 \\
\hline Thermo & YBDE18 & 18 & 0.062 & 0.013 & 0.028 & 0.040 & 0.014 \\
\hline \multicolumn{2}{|l}{ Total WTMAD2 } & $\mathbf{6 . 5 0 2}$ & $\mathbf{6 . 5 5 1}$ & $\mathbf{3 . 9 5 9}$ & $\mathbf{3 . 2 8 6}$ & $\mathbf{2 . 1 8 6}$ \\
\hline
\end{tabular}

Table S12: Integration grid convergence

\begin{tabular}{|c|c|c|c|c|c|c|c|c|c|c|}
\hline \multirow{3}{*}{$\begin{array}{l}\text { Serial } \\
\text { No. }\end{array}$} & \multirow{3}{*}{ Set Name } & \multicolumn{9}{|c|}{ AWTMAD2(kcal/mol) } \\
\hline & & \multicolumn{2}{|c|}{$\omega B 97 X-V$} & \multicolumn{3}{|c|}{$\omega \mathrm{B97M-V}$} & \multicolumn{2}{|c|}{ B3LYP-D3 } & \multicolumn{2}{|c|}{ DSD-SCAN0-2 } \\
\hline & & SG2 & SG3 & SG2 & SG3 & grid $=150,590$ & SG2 & SG3 & SG3 & grid $=150,590$ \\
\hline 1 & ACONF & 0.011 & 0.008 & 0.023 & 0.021 & 0.018 & 0.027 & 0.024 & 0.026 & 0.022 \\
\hline 2 & ADIM6 & 0.008 & 0.009 & 0.005 & 0.007 & 0.007 & 0.010 & 0.012 & 0.049 & 0.041 \\
\hline 3 & AHB21 & 0.054 & 0.012 & 0.047 & 0.007 & 0.008 & 0.018 & 0.017 & 0.013 & 0.013 \\
\hline 4 & AL2X6 & 0.008 & 0.008 & 0.008 & 0.008 & 0.008 & 0.016 & 0.016 & 0.002 & 0.001 \\
\hline 5 & ALK8 & 0.004 & 0.004 & 0.012 & 0.012 & 0.012 & 0.012 & 0.012 & 0.003 & 0.003 \\
\hline 6 & ALKBDE10 & 0.015 & 0.015 & 0.014 & 0.014 & 0.014 & 0.016 & 0.016 & 0.009 & 0.009 \\
\hline 7 & AMINO20X4 & 0.250 & 0.243 & 0.239 & 0.231 & 0.236 & 0.276 & 0.258 & 0.155 & 0.154 \\
\hline 8 & BH76RC & 0.096 & 0.096 & 0.061 & 0.061 & 0.061 & 0.117 & 0.117 & 0.063 & 0.063 \\
\hline 9 & $\mathrm{BH} 76$ & 0.285 & 0.286 & 0.257 & 0.258 & 0.258 & 0.847 & 0.849 & 0.311 & 0.309 \\
\hline 10 & BHDIV10 & 0.007 & 0.007 & 0.011 & 0.011 & 0.011 & 0.027 & 0.027 & 0.006 & 0.006 \\
\hline 11 & BHPERI & 0.099 & 0.099 & 0.054 & 0.053 & 0.053 & 0.055 & 0.057 & 0.064 & 0.067 \\
\hline 12 & BHROT27 & 0.049 & 0.049 & 0.037 & 0.038 & 0.035 & 0.075 & 0.069 & 0.032 & 0.032 \\
\hline 13 & BSR36 & 0.184 & 0.184 & 0.025 & 0.033 & 0.043 & 0.291 & 0.301 & 0.044 & 0.037 \\
\hline 14 & BUT14DIOL & 0.054 & 0.052 & 0.039 & 0.056 & 0.052 & 0.288 & 0.299 & 0.069 & 0.064 \\
\hline 15 & C60ISO & 0.047 & 0.047 & 0.040 & 0.041 & 0.041 & 0.007 & 0.008 & 0.023 & 0.023 \\
\hline 16 & CARBHB12 & 0.027 & 0.027 & 0.015 & 0.014 & 0.015 & 0.076 & 0.072 & 0.022 & 0.022 \\
\hline 17 & CDIE20 & 0.115 & 0.115 & 0.109 & 0.110 & 0.111 & 0.187 & 0.187 & 0.063 & 0.063 \\
\hline 18 & CHB6 & 0.008 & 0.008 & 0.008 & 0.008 & 0.008 & 0.012 & 0.012 & 0.009 & 0.009 \\
\hline 19 & DARC & 0.071 & 0.070 & 0.012 & 0.012 & 0.012 & 0.128 & 0.127 & 0.039 & 0.041 \\
\hline 20 & DC13 & 0.067 & 0.059 & 0.049 & 0.049 & 0.049 & 0.093 & 0.094 & 0.030 & 0.031 \\
\hline 21 & DIPCS10 & 0.002 & 0.002 & 0.003 & 0.003 & 0.003 & 0.003 & 0.003 & 0.002 & 0.002 \\
\hline 22 & FH51 & 0.144 & 0.144 & 0.067 & 0.067 & 0.066 & 0.160 & 0.159 & 0.063 & 0.064 \\
\hline 23 & G21EA & 0.114 & 0.073 & 0.111 & 0.064 & 0.064 & 0.065 & 0.063 & 0.081 & 0.079 \\
\hline 24 & G21IP & 0.016 & 0.016 & 0.015 & 0.015 & 0.015 & 0.020 & 0.020 & 0.011 & 0.011 \\
\hline 25 & G2RC & 0.073 & 0.073 & 0.038 & 0.038 & 0.037 & 0.051 & 0.051 & 0.029 & 0.029 \\
\hline 26 & HAL59 & 0.150 & 0.150 & 0.140 & 0.140 & 0.136 & 0.332 & 0.301 & 0.153 & 0.116 \\
\hline 27 & HEAVY28 & 0.144 & 0.154 & 0.149 & 0.156 & 0.156 & 0.334 & 0.312 & 0.098 & 0.062 \\
\hline 28 & HEAVYSB11 & 0.010 & 0.010 & 0.019 & 0.019 & 0.019 & 0.022 & 0.022 & 0.012 & 0.007 \\
\hline 29 & ICONF & 0.054 & 0.050 & 0.03 & 0.028 & 0.029 & 0.063 & 0.055 & 0.020 & 0.020 \\
\hline 30 & IDISP & 0.042 & 0.041 & 0.027 & 0.026 & 0.026 & 0.057 & 0.057 & 0.018 & 0.019 \\
\hline 31 & IL16 & 0.002 & 0.005 & 0.002 & 0.004 & 0.004 & 0.003 & 0.004 & 0.003 & 0.002 \\
\hline 32 & INV24 & 0.049 & 0.048 & 0.038 & 0.038 & 0.038 & 0.030 & 0.029 & 0.021 & 0.023 \\
\hline 33 & ISO34 & 0.103 & 0.103 & 0.056 & 0.055 & 0.055 & 0.156 & 0.155 & 0.046 & 0.046 \\
\hline 34 & ISOL24 & 0.124 & 0.123 & 0.068 & 0.068 & 0.067 & 0.238 & 0.237 & 0.040 & 0.041 \\
\hline 35 & MB16-43 & 0.115 & 0.115 & 0.053 & 0.052 & 0.051 & 0.084 & 0.084 & 0.022 & 0.023 \\
\hline 36 & MCONF & 0.083 & 0.090 & 0.113 & 0.152 & 0.149 & 0.120 & 0.087 & 0.093 & 0.116 \\
\hline 37 & NBPRC & 0.010 & 0.010 & 0.006 & 0.005 & 0.005 & 0.007 & 0.007 & 0.004 & 0.005 \\
\hline 38 & PA26 & 0.014 & 0.014 & 0.007 & 0.007 & 0.007 & 0.015 & 0.015 & 0.008 & 0.008 \\
\hline 39 & PAREL & 0.104 & 0.104 & 0.094 & 0.095 & 0.098 & 0.192 & 0.191 & 0.072 & 0.073 \\
\hline
\end{tabular}




\begin{tabular}{|l|l|l|l|l|l|l|l|l|l|l|}
\hline 40 & PCONF21 & 0.130 & 0.128 & 0.275 & 0.267 & 0.263 & 0.229 & 0.228 & 0.082 & 0.090 \\
\hline 41 & PNICO23 & 0.042 & 0.037 & 0.057 & 0.053 & 0.053 & 0.111 & 0.111 & 0.024 & 0.022 \\
\hline 42 & PX13 & 0.038 & 0.038 & 0.029 & 0.029 & 0.028 & 0.065 & 0.065 & 0.017 & 0.017 \\
\hline 43 & RC21 & 0.079 & 0.079 & 0.039 & 0.039 & 0.039 & 0.059 & 0.059 & 0.065 & 0.064 \\
\hline 44 & RG18 & 0.299 & 0.082 & 0.104 & 0.072 & 0.070 & 0.459 & 0.157 & 0.120 & 0.110 \\
\hline 45 & RSE43 & 0.205 & 0.207 & 0.174 & 0.173 & 0.166 & 0.367 & 0.372 & 0.362 & 0.360 \\
\hline 46 & S22 & 0.027 & 0.026 & 0.026 & 0.028 & 0.027 & 0.043 & 0.042 & 0.024 & 0.026 \\
\hline 47 & S66 & 0.058 & 0.056 & 0.070 & 0.070 & 0.066 & 0.143 & 0.152 & 0.105 & 0.086 \\
\hline 48 & SCONF & 0.020 & 0.021 & 0.033 & 0.030 & 0.024 & 0.044 & 0.043 & 0.008 & 0.005 \\
\hline 49 & SIE4X4 & 0.206 & 0.206 & 0.193 & 0.193 & 0.193 & 0.324 & 0.324 & 0.032 & 0.033 \\
\hline 50 & TAUT15 & 0.133 & 0.134 & 0.058 & 0.058 & 0.062 & 0.226 & 0.22 & 0.072 & 0.072 \\
\hline 51 & UPU23 & 0.081 & 0.097 & 0.067 & 0.084 & 0.083 & 0.092 & 0.097 & 0.059 & 0.060 \\
\hline 52 & W4-11 & 0.049 & 0.048 & 0.036 & 0.036 & 0.036 & 0.056 & 0.056 & 0.045 & 0.046 \\
\hline 53 & WATER27 & 0.057 & 0.012 & 0.056 & 0.005 & 0.007 & 0.049 & 0.048 & 0.013 & 0.011 \\
\hline 54 & WCPT18 & 0.034 & 0.034 & 0.028 & 0.028 & 0.028 & 0.045 & 0.046 & 0.014 & 0.014 \\
\hline 55 & YBDE18 & 0.028 & 0.028 & 0.040 & 0.040 & 0.040 & 0.062 & 0.062 & 0.028 & 0.029 \\
\hline
\end{tabular}

Table S13: Performance Comparison for the WTMAD2 components (kcal/mol) between DSD-PBEP86D3(BJ), DSD-PBEP86-D4, revDSD-PBEP86-D3(BJ), revDSD-PBE86-noFC (core + valence), and revDSD-PBEP86-D4

\begin{tabular}{|c|c|c|c|c|c|c|c|}
\hline \multirow{2}{*}{$\begin{array}{l}\text { Serial } \\
\text { No }\end{array}$} & \multirow[t]{2}{*}{ Subset Name } & \multicolumn{6}{|c|}{ AWTMAD2(kcal/mol) } \\
\hline & & $\begin{array}{l}\text { DSD- } \\
\text { PBEP86- } \\
\text { D3(BJ) }\end{array}$ & $\begin{array}{c}\text { DSD- } \\
\text { PBEP86-D4 }\end{array}$ & $\begin{array}{c}\text { revDSD- } \\
\text { PBEP86- } \\
\text { D3(BJ) }\end{array}$ & $\begin{array}{c}\text { revDSD- } \\
\text { PBEP86(noFC) } \\
\text {-D3(BJ) }\end{array}$ & $\begin{array}{c}\text { revDSD- } \\
\text { PBEP86- } \\
\text { NL }\end{array}$ & $\begin{array}{c}\text { revDSD- } \\
\text { PBEP86-D4 }\end{array}$ \\
\hline 1 & ACONF & 0.012 & 0.039 & 0.013 & 0.008 & 0.012 & 0.009 \\
\hline 2 & ADIM6 & 0.007 & 0.034 & 0.021 & 0.023 & 0.018 & 0.019 \\
\hline 3 & AHB21 & 0.016 & 0.011 & 0.010 & 0.011 & 0.010 & 0.008 \\
\hline 4 & AL2X6 & 0.004 & 0.012 & 0.003 & 0.002 & 0.003 & 0.011 \\
\hline 5 & ALK8 & 0.006 & 0.009 & 0.004 & 0.009 & 0.005 & 0.010 \\
\hline 6 & ALKBDE10 & 0.011 & 0.012 & 0.011 & 0.011 & 0.011 & 0.011 \\
\hline 7 & AMINO20X4 & 0.147 & 0.163 & 0.153 & 0.165 & 0.156 & 0.135 \\
\hline 8 & BH76RC & 0.049 & 0.048 & 0.053 & 0.051 & 0.055 & 0.055 \\
\hline 9 & $\mathrm{BH} 76$ & 0.197 & 0.181 & 0.181 & 0.177 & 0.182 & 0.190 \\
\hline 10 & BHDIV10 & 0.014 & 0.011 & 0.008 & 0.008 & 0.007 & 0.007 \\
\hline 11 & BHPERI & 0.115 & 0.065 & 0.038 & 0.035 & 0.032 & 0.040 \\
\hline 12 & BHROT27 & 0.032 & 0.029 & 0.020 & 0.024 & 0.020 & 0.016 \\
\hline 13 & BSR36 & 0.026 & 0.137 & 0.129 & 0.093 & 0.131 & 0.117 \\
\hline 14 & BUT14DIOL & 0.082 & 0.051 & 0.045 & 0.039 & 0.047 & 0.050 \\
\hline 15 & C60ISO & 0.023 & 0.024 & 0.019 & 0.019 & 0.018 & 0.022 \\
\hline 16 & CARBHB12 & 0.052 & 0.034 & 0.032 & 0.030 & 0.031 & 0.027 \\
\hline 17 & CDIE20 & 0.087 & 0.101 & 0.065 & 0.072 & 0.062 & 0.064 \\
\hline 18 & CHB6 & 0.011 & 0.006 & 0.010 & 0.009 & 0.010 & 0.006 \\
\hline 19 & DARC & 0.024 & 0.013 & 0.009 & 0.010 & 0.008 & 0.009 \\
\hline 20 & DC13 & 0.022 & 0.021 & 0.017 & 0.030 & 0.016 & 0.016 \\
\hline 21 & DIPCS10 & 0.003 & 0.003 & 0.003 & 0.003 & 0.003 & 0.003 \\
\hline 22 & FH51 & 0.052 & 0.046 & 0.043 & 0.043 & 0.044 & 0.045 \\
\hline 23 & G21EA & 0.068 & 0.067 & 0.074 & 0.080 & 0.077 & 0.072 \\
\hline 24 & G21IP & 0.011 & 0.011 & 0.012 & 0.012 & 0.012 & 0.012 \\
\hline 25 & G2RC & 0.036 & 0.030 & 0.030 & 0.030 & 0.030 & 0.028 \\
\hline 26 & HAL59 & 0.242 & 0.115 & 0.141 & 0.155 & 0.142 & 0.100 \\
\hline 27 & HEAVY28 & 0.230 & 0.090 & 0.116 & 0.150 & 0.122 & 0.097 \\
\hline 28 & HEAVYSB11 & 0.007 & 0.011 & 0.006 & 0.008 & 0.007 & 0.011 \\
\hline 29 & ICONF & 0.018 & 0.033 & 0.019 & 0.028 & 0.019 & 0.021 \\
\hline 30 & IDISP & 0.020 & 0.014 & 0.009 & 0.009 & 0.008 & 0.008 \\
\hline 31 & IL16 & 0.001 & 0.003 & 0.003 & 0.003 & 0.004 & 0.002 \\
\hline 32 & INV24 & 0.020 & 0.019 & 0.018 & 0.018 & 0.018 & 0.018 \\
\hline 33 & ISO34 & 0.035 & 0.043 & 0.032 & 0.031 & 0.031 & 0.033 \\
\hline 34 & ISOL24 & 0.048 & 0.057 & 0.045 & 0.042 & 0.044 & 0.045 \\
\hline 35 & MB16-43 & 0.018 & 0.035 & 0.027 & 0.036 & 0.029 & 0.051 \\
\hline 36 & MCONF & 0.156 & 0.044 & 0.061 & 0.063 & 0.064 & 0.050 \\
\hline 37 & NBPRC & 0.008 & 0.005 & 0.004 & 0.004 & 0.004 & 0.004 \\
\hline
\end{tabular}




\begin{tabular}{|l|l|l|l|l|l|l|l|}
\hline 38 & PA26 & 0.006 & 0.005 & 0.009 & 0.010 & 0.009 & 0.007 \\
\hline 39 & PAREL & 0.080 & 0.077 & 0.060 & 0.061 & 0.057 & 0.061 \\
\hline 40 & PCONF21 & 0.129 & 0.134 & 0.082 & 0.086 & 0.079 & 0.058 \\
\hline 41 & PNICO23 & 0.099 & 0.018 & 0.040 & 0.031 & 0.042 & 0.020 \\
\hline 42 & PX13 & 0.036 & 0.033 & 0.023 & 0.019 & 0.021 & 0.024 \\
\hline 43 & RC21 & 0.033 & 0.031 & 0.032 & 0.032 & 0.032 & 0.033 \\
\hline 44 & RG18 & 0.085 & 0.130 & 0.103 & 0.091 & 0.101 & 0.102 \\
\hline 45 & RSE43 & 0.146 & 0.144 & 0.169 & 0.160 & 0.172 & 0.176 \\
\hline 46 & S22 & 0.048 & 0.017 & 0.020 & 0.020 & 0.020 & 0.015 \\
\hline 47 & S66 & 0.149 & 0.095 & 0.067 & 0.073 & 0.064 & 0.073 \\
\hline 48 & SCONF & 0.020 & 0.010 & 0.013 & 0.013 & 0.015 & 0.008 \\
\hline 49 & SIE4X4 & 0.092 & 0.090 & 0.089 & 0.088 & 0.087 & 0.089 \\
\hline 50 & TAUT15 & 0.088 & 0.084 & 0.092 & 0.091 & 0.096 & 0.097 \\
\hline 51 & UPU23 & 0.065 & 0.067 & 0.069 & 0.072 & 0.070 & 0.069 \\
\hline 52 & W4-11 & 0.035 & 0.042 & 0.042 & 0.043 & 0.046 & 0.042 \\
\hline 53 & WATER27 & 0.027 & 0.009 & 0.007 & 0.007 & 0.007 & 0.008 \\
\hline 54 & WCPT18 & 0.034 & 0.029 & 0.019 & 0.016 & 0.017 & 0.019 \\
\hline 55 & YBDE18 & 0.020 & 0.012 & 0.011 & 0.011 & 0.011 & 0.010 \\
\hline Total WTMAD2 & $\mathbf{3 . 1 0 0}$ & $\mathbf{2 . 6 4 9}$ & $\mathbf{2 . 4 2 9}$ & $\mathbf{2 . 4 6 3}$ & $\mathbf{2 . 4 3 5}$ & $\mathbf{2 . 3 3 2}$ \\
\hline
\end{tabular}

Table S14: Benefit of correlating the inner-shell orbitals for DSD-BLYP: component-wise analysis

\begin{tabular}{|c|c|c|c|c|}
\hline \multirow{2}{*}{$\begin{array}{l}\text { Serial } \\
\text { No. }\end{array}$} & \multirow[t]{2}{*}{ Set Name } & \multirow{2}{*}{$\begin{array}{l}\text { No. of } \\
\text { Points }\end{array}$} & \multicolumn{2}{|c|}{$\Delta$ WTMAD2(kcal/mol) } \\
\hline & & & revDSD-BLYP with frozen cores & revDSD-BLYP without frozen cores \\
\hline 1 & ACONF & 15 & 0.013 & 0.010 \\
\hline 2 & ADIM6 & 6 & 0.022 & 0.026 \\
\hline 3 & AHB21 & 21 & 0.010 & 0.011 \\
\hline 4 & AL2X6 & 6 & 0.004 & 0.003 \\
\hline 5 & ALK8 & 8 & 0.011 & 0.009 \\
\hline 6 & ALKBDE10 & 10 & 0.009 & 0.009 \\
\hline 7 & AMINO20X4 & 80 & 0.143 & 0.164 \\
\hline 8 & BH76RC & 30 & 0.056 & 0.053 \\
\hline 9 & $\mathrm{BH} 76$ & 76 & 0.194 & 0.186 \\
\hline 10 & BHDIV10 & 10 & 0.008 & 0.008 \\
\hline 11 & BHPERI & 26 & 0.037 & 0.032 \\
\hline 12 & BHROT27 & 27 & 0.030 & 0.033 \\
\hline 13 & BSR36 & 36 & 0.088 & 0.056 \\
\hline 14 & BUT14DIOL & 64 & 0.036 & 0.035 \\
\hline 15 & C60ISO & 9 & 0.019 & 0.019 \\
\hline 16 & CARBHB12 & 12 & 0.027 & 0.026 \\
\hline 17 & CDIE20 & 20 & 0.065 & 0.077 \\
\hline 18 & CHB6 & 6 & 0.014 & 0.012 \\
\hline 19 & DARC & 14 & 0.029 & 0.028 \\
\hline 20 & DC13 & 13 & 0.031 & 0.028 \\
\hline 21 & DIPCS10 & 10 & 0.002 & 0.002 \\
\hline 22 & FH51 & 51 & 0.049 & 0.052 \\
\hline 23 & G21EA & 25 & 0.085 & 0.088 \\
\hline 24 & G21IP & 36 & 0.011 & 0.011 \\
\hline 25 & G2RC & 25 & 0.017 & 0.017 \\
\hline 26 & HAL59 & 59 & 0.138 & 0.147 \\
\hline 27 & HEAVY28 & 28 & 0.077 & 0.087 \\
\hline 28 & HEAVYSB11 & 11 & 0.009 & 0.012 \\
\hline 29 & ICONF & 17 & 0.025 & 0.027 \\
\hline 30 & IDISP & 6 & 0.011 & 0.009 \\
\hline 31 & IL16 & 16 & 0.003 & 0.003 \\
\hline 32 & INV24 & 24 & 0.023 & 0.023 \\
\hline 33 & ISO34 & 34 & 0.057 & 0.054 \\
\hline 34 & ISOL24 & 24 & 0.082 & 0.077 \\
\hline 35 & MB16-43 & 43 & 0.024 & 0.036 \\
\hline 36 & MCONF & 51 & 0.083 & 0.088 \\
\hline 37 & NBPRC & 6 & 0.003 & 0.003 \\
\hline 38 & PA26 & 26 & 0.009 & 0.009 \\
\hline
\end{tabular}




\begin{tabular}{|l|l|l|l|l|}
\hline 39 & PAREL & 20 & 0.076 & 0.076 \\
\hline 40 & PCONF21 & 18 & 0.085 & 0.086 \\
\hline 41 & PNICO23 & 23 & 0.028 & 0.019 \\
\hline 42 & PX13 & 13 & 0.011 & 0.008 \\
\hline 43 & RC21 & 21 & 0.038 & 0.039 \\
\hline 44 & RG18 & 18 & 0.131 & 0.110 \\
\hline 45 & RSE43 & 43 & 0.142 & 0.136 \\
\hline 46 & S22 & 22 & 0.017 & 0.017 \\
\hline 47 & S66 & 66 & 0.074 & 0.076 \\
\hline 48 & SCONF & 17 & 0.009 & 0.011 \\
\hline 49 & SIE4X4 & 16 & 0.094 & 0.093 \\
\hline 50 & TAUT15 & 15 & 0.080 & 0.081 \\
\hline 51 & UPU23 & 23 & 0.068 & 0.071 \\
\hline 52 & W4-11 & 140 & 0.043 & 0.045 \\
\hline 53 & WATER27 & 27 & 0.010 & 0.008 \\
\hline 54 & WCPT18 & 18 & 0.015 & 0.014 \\
\hline 55 & YBDE18 & 18 & 0.013 & 0.013 \\
\hline \multicolumn{7}{|l}{ WTMAD2 } & $\mathbf{2 . 4 8 4}$ & $\mathbf{2 . 4 7 0}$ \\
\hline
\end{tabular}

Table S15: Comparison of B3LYP-D3(BJ), PBE0-D3(BJ), and revPBE0-D3(BJ) for the WTMAD2 components

\begin{tabular}{|c|c|c|c|c|c|c|}
\hline \multirow{2}{*}{$\begin{array}{l}\text { Serial } \\
\text { No }\end{array}$} & \multirow[t]{2}{*}{ Type } & \multirow[t]{2}{*}{ Set Name } & \multirow{2}{*}{$\begin{array}{l}\text { No. of } \\
\text { Points }\end{array}$} & \multicolumn{3}{|c|}{$\Delta$ WTMAD2(kcal/mol) } \\
\hline & & & & B3LYP-D3(BJ) & PBE0-D3(BJ) & revPBE0-D3(BJ) \\
\hline 1 & Conformer & ACONF & 15 & 0.024 & 0.017 & 0.015 \\
\hline 2 & Intermol & ADIM6 & 6 & 0.012 & 0.004 & 0.005 \\
\hline 3 & Intermol & AHB21 & 21 & 0.017 & 0.048 & 0.020 \\
\hline 4 & Thermo & AL2X6 & 6 & 0.016 & 0.009 & 0.010 \\
\hline 5 & Thermo & ALK8 & 8 & 0.012 & 0.023 & 0.024 \\
\hline 6 & Thermo & ALKBDE10 & 10 & 0.016 & 0.021 & 0.030 \\
\hline 7 & Conformer & AMINO20X4 & 80 & 0.258 & 0.345 & 0.377 \\
\hline 8 & Thermo & BH76RC & 30 & 0.117 & 0.131 & 0.105 \\
\hline 9 & Barrier & BH76 & 76 & 0.849 & 0.704 & 0.547 \\
\hline 10 & Barrier & BHDIV10 & 10 & 0.027 & 0.040 & 0.035 \\
\hline 11 & Barrier & BHPERI & 26 & 0.057 & 0.153 & 0.151 \\
\hline 12 & Barrier & BHROT27 & 27 & 0.069 & 0.095 & 0.076 \\
\hline 13 & REACLarge & BSR36 & 36 & 0.301 & 0.294 & 0.187 \\
\hline 14 & Conformer & BUT14DIOL & 64 & 0.299 & 0.210 & 0.203 \\
\hline 15 & REACLarge & C60ISO & 9 & 0.008 & 0.009 & 0.009 \\
\hline 16 & Intermol & CARBHB12 & 12 & 0.072 & 0.110 & 0.070 \\
\hline 17 & REACLarge & CDIE20 & 20 & 0.187 & 0.231 & 0.202 \\
\hline 18 & Intermol & CHB6 & 6 & 0.012 & 0.012 & 0.010 \\
\hline 19 & REACLarge & DARC & 14 & 0.127 & 0.061 & 0.052 \\
\hline 20 & Thermo & DC13 & 13 & 0.094 & 0.072 & 0.057 \\
\hline 21 & Thermo & DIPCS10 & 10 & 0.003 & 0.002 & 0.002 \\
\hline 22 & Thermo & FH51 & 51 & 0.159 & 0.173 & 0.134 \\
\hline 23 & Thermo & G21EA & 25 & 0.063 & 0.083 & 0.105 \\
\hline 24 & Thermo & G21IP & 36 & 0.020 & 0.020 & 0.021 \\
\hline 25 & Thermo & G2RC & 25 & 0.051 & 0.125 & 0.101 \\
\hline 26 & Intermol & HAL59 & 59 & 0.301 & 0.299 & 0.238 \\
\hline 27 & Intermol & HEAVY28 & 28 & 0.312 & 0.261 & 0.210 \\
\hline 28 & Thermo & HEAVYSB11 & 11 & 0.022 & 0.010 & 0.020 \\
\hline 29 & Conformer & ICONF & 17 & 0.055 & 0.056 & 0.046 \\
\hline 30 & Conformer & IDISP & 6 & 0.057 & 0.025 & 0.023 \\
\hline 31 & Intermol & IL16 & 16 & 0.004 & 0.002 & 0.004 \\
\hline 32 & Barrier & INV24 & 24 & 0.029 & 0.033 & 0.038 \\
\hline 33 & REACLarge & ISO34 & 34 & 0.155 & 0.126 & 0.107 \\
\hline 34 & REACLarge & ISOL24 & 24 & 0.237 & 0.088 & 0.070 \\
\hline 35 & REACLarge & MB16-43 & 43 & 0.084 & 0.055 & 0.056 \\
\hline 36 & Conformer & MCONF & 51 & 0.087 & 0.106 & 0.110 \\
\hline 37 & Thermo & NBPRC & 6 & 0.007 & 0.023 & 0.023 \\
\hline 38 & Thermo & PA26 & 26 & 0.015 & 0.015 & 0.026 \\
\hline 39 & REACLarge & PAREL & 20 & 0.191 & 0.197 & 0.159 \\
\hline 40 & Conformer & PCONF21 & 18 & 0.228 & 0.382 & 0.235 \\
\hline
\end{tabular}




\begin{tabular}{|c|c|c|c|c|c|c|}
\hline 41 & Intermol & PNICO23 & 23 & 0.111 & 0.196 & 0.149 \\
\hline 42 & Barrier & PX13 & 13 & 0.065 & 0.097 & 0.060 \\
\hline 43 & Thermo & $\mathrm{RC} 21$ & 21 & 0.059 & 0.126 & 0.095 \\
\hline 44 & Intermol & RG18 & 18 & 0.157 & 0.106 & 0.054 \\
\hline 45 & REACLarge & RSE43 & 43 & 0.372 & 0.309 & 0.212 \\
\hline 46 & Intermol & S22 & 22 & 0.042 & 0.059 & 0.032 \\
\hline 47 & Intermol & S66 & 66 & 0.152 & 0.171 & 0.083 \\
\hline 48 & Conformer & SCONF & 17 & 0.043 & 0.038 & 0.040 \\
\hline 49 & Thermo & SIE4X4 & 16 & 0.324 & 0.259 & 0.254 \\
\hline 50 & Thermo & TAUT15 & 15 & 0.220 & 0.211 & 0.172 \\
\hline 51 & Conformer & UPU23 & 23 & 0.097 & 0.087 & 0.084 \\
\hline 52 & Thermo & W4-11 & 140 & 0.056 & 0.061 & 0.158 \\
\hline 53 & Intermol & WATER27 & 27 & 0.048 & 0.067 & 0.038 \\
\hline 54 & Barrier & WCPT18 & 18 & 0.046 & 0.085 & 0.055 \\
\hline 55 & Thermo & YBDE18 & 18 & 0.062 & 0.013 & 0.031 \\
\hline \multicolumn{4}{|c|}{ Total WTMAD2 } & 6.502 & 6.551 & 5.429 \\
\hline
\end{tabular}

Table S16: Sensitivity of WTMAD2 to the damping function scale parameter $\mathbf{a}_{2}$

\begin{tabular}{|c|c|c|c|c|c|c|c|}
\hline \multirow[t]{3}{*}{ Functionals } & \multirow{3}{*}{$\begin{array}{c}\text { Optimized } \mathbf{a}_{2} \\
\text { WTMAD2 }\end{array}$} & \multicolumn{6}{|c|}{ Fixed a2 } \\
\hline & & \multicolumn{2}{|c|}{$a_{2}=5.2$} & \multicolumn{2}{|c|}{$a_{2}=5.5$} & \multicolumn{2}{|c|}{$\mathrm{a}_{2}=\mathbf{5 . 7 5}$} \\
\hline & & WTMAD2 & $\begin{array}{l}\text { WTMAD2 } \\
\text { difference }^{*}\end{array}$ & WTMAD2 & $\begin{array}{l}\text { WTMAD2 } \\
\text { difference }^{*}\end{array}$ & WTMAD2 & $\begin{array}{l}\text { WTMAD2 } \\
\text { difference }^{*}\end{array}$ \\
\hline \multicolumn{8}{|c|}{ without inner-shell correlation } \\
\hline DSD-BLYP-D3(BJ) & 2.484 & 2.484 & 0.000 & 2.491 & 0.007 & 2.508 & 0.024 \\
\hline DSD-PBEB95-D3(BJ) & 2.845 & 2.840 & -0.005 & 2.846 & 0.001 & 2.851 & 0.006 \\
\hline DSD-PBEP86-D3(BJ) & 2.429 & 2.412 & -0.017 & 2.422 & -0.007 & 2.437 & 0.008 \\
\hline DSD-PBE-D3(BJ) & 2.738 & 2.716 & -0.022 & 2.722 & -0.016 & 2.737 & -0.001 \\
\hline DSD-SCAN $_{50}-\mathrm{D} 3(\mathrm{BJ})$ & 3.104 & 3.168 & 0.064 & 3.138 & 0.034 & 3.119 & 0.015 \\
\hline DSD-SCAN $_{55}-\mathrm{D} 3(\mathrm{BJ})$ & 2.892 & 2.944 & 0.052 & 2.919 & 0.027 & 2.904 & 0.012 \\
\hline DSD-SCAN $_{58}-\mathrm{D} 3(\mathrm{BJ})$ & 2.785 & 2.820 & 0.035 & 2.801 & 0.016 & 2.788 & 0.003 \\
\hline DSD-SCAN $_{63}-\mathrm{D} 3(\mathrm{BJ})$ & 2.691 & 2.717 & 0.026 & 2.701 & 0.010 & 2.693 & 0.002 \\
\hline DSD-SCAN $_{66}-\mathrm{D} 3(\mathrm{BJ})$ & 2.662 & 2.684 & 0.022 & 2.672 & 0.010 & 2.666 & 0.004 \\
\hline DSD-SCAN $_{69}-\mathrm{D} 3(\mathrm{BJ})$ & 2.659 & 2.672 & 0.013 & 2.663 & 0.004 & 2.658 & -0.001 \\
\hline DSD-SCAN $_{74-D 3(B J)}$ & 2.688 & 2.696 & 0.008 & 2.689 & 0.001 & 2.685 & -0.003 \\
\hline DSD-SCAN0-2-D3(BJ) & 2.800 & 2.806 & 0.006 & 2.803 & 0.003 & 2.800 & 0.000 \\
\hline DSD-TPSS-D3(BJ) & 2.954 & 2.962 & 0.008 & 2.954 & 0.000 & 2.957 & 0.003 \\
\hline \multicolumn{8}{|c|}{ with inner-shell correlation } \\
\hline DSD-BLYP-D3(BJ) & 2.470 & 2.450 & -0.020 & 2.475 & 0.005 & 2.504 & 0.034 \\
\hline DSD-PBEB95-D3(BJ) & 2.866 & 2.851 & -0.015 & 2.865 & -0.001 & 2.873 & 0.007 \\
\hline DSD-PBE-D3(BJ) & 2.718 & 2.719 & 0.001 & 2.735 & 0.017 & 2.755 & 0.037 \\
\hline DSD-PBEP86-D3(BJ) & 2.463 & 2.448 & -0.015 & 2.465 & 0.002 & 2.482 & 0.019 \\
\hline DSD-SCAN $_{50}-\mathrm{D} 3(\mathrm{BJ})$ & 3.071 & 3.112 & 0.041 & 3.091 & 0.020 & 3.080 & 0.009 \\
\hline DSD-SCAN $_{55}$-D3(BJ) & 2.860 & 2.890 & 0.030 & 2.877 & 0.017 & 2.868 & 0.008 \\
\hline DSD-SCAN $_{58}-\mathrm{D} 3(\mathrm{BJ})$ & 2.747 & 2.769 & 0.022 & 2.760 & 0.013 & 2.754 & 0.007 \\
\hline DSD-SCAN $_{63}-\mathrm{D} 3(\mathrm{BJ})$ & 2.666 & 2.675 & 0.009 & 2.669 & 0.003 & 2.665 & -0.001 \\
\hline DSD-SCAN $_{66}-\mathrm{D} 3(\mathrm{BJ})$ & 2.649 & 2.651 & 0.002 & 2.651 & 0.002 & 2.649 & 0.000 \\
\hline DSD-SCAN $_{69}-\mathrm{D} 3(\mathrm{BJ})$ & 2.660 & 2.665 & 0.005 & 2.662 & 0.002 & 2.661 & 0.001 \\
\hline DSD-SCAN $_{74-D 3(B J)}$ & 2.727 & 2.727 & 0.000 & 2.727 & 0.000 & 2.726 & -0.001 \\
\hline DSD-SCAN0-2-D3(BJ) & 2.880 & 2.879 & -0.001 & 2.880 & 0.000 & 2.880 & 0.000 \\
\hline
\end{tabular}

*WTMAD2 difference $=\left(\right.$ WTMAD2 for fixed $\mathrm{a}_{2}-\mathrm{WTMAD} 2$ for optimized $\left.\mathrm{a}_{2}\right)$. All WTMAD2 values are in kcal/mol. 
Table S17: Comparison of revDSD-PBEP86-D3(BJ), revDSD-PBEP86-D4, revDSD-PBEP86-D4(noFC), and xrevDSD-PBEP86-D4(noFC) for the WTMAD2 components over whole 55 subsets.

\begin{tabular}{|c|c|c|c|c|c|c|}
\hline \multirow{2}{*}{$\begin{array}{l}\text { Serial } \\
\text { No }\end{array}$} & \multirow[t]{2}{*}{ Type } & \multirow[t]{2}{*}{ Subset Name } & \multicolumn{4}{|c|}{$\Delta$ WTMAD2(kcal/mol) } \\
\hline & & & $\begin{array}{c}\text { revDSD- } \\
\text { PBEP86- } \\
\text { D3(BJ) }\end{array}$ & $\begin{array}{c}\text { revDSD- } \\
\text { PBEP86-D4 }\end{array}$ & $\begin{array}{c}\text { revDSD- } \\
\text { PBEP86(noFC)-D4 }\end{array}$ & $\begin{array}{c}\text { xrevDSD- } \\
\text { PBEP86(noFC)-D4 }\end{array}$ \\
\hline 1 & Conformer & ACONF & 0.013 & 0.009 & 0.005 & 0.008 \\
\hline 2 & Intermol & ADIM6 & 0.021 & 0.019 & 0.021 & 0.018 \\
\hline 3 & Intermol & AHB21 & 0.010 & 0.008 & 0.010 & 0.010 \\
\hline 4 & Thermo & AL2X6 & 0.003 & 0.011 & 0.008 & 0.006 \\
\hline 5 & Thermo & ALK8 & 0.004 & 0.010 & 0.007 & 0.006 \\
\hline 6 & Thermo & ALKBDE10 & 0.011 & 0.011 & 0.012 & 0.013 \\
\hline 7 & Conformer & AMINO20X4 & 0.153 & 0.135 & 0.153 & 0.145 \\
\hline 8 & Thermo & BH76RC & 0.053 & 0.055 & 0.051 & 0.053 \\
\hline 9 & Barrier & BH76 & 0.181 & 0.190 & 0.183 & 0.173 \\
\hline 10 & Barrier & BHDIV10 & 0.008 & 0.007 & 0.007 & 0.007 \\
\hline 11 & Barrier & BHPERI & 0.038 & 0.040 & 0.033 & 0.021 \\
\hline 12 & Barrier & BHROT27 & 0.020 & 0.016 & 0.020 & 0.020 \\
\hline 13 & REACLarge & BSR36 & 0.129 & 0.117 & 0.082 & 0.074 \\
\hline 14 & Conformer & BUT14DIOL & 0.045 & 0.050 & 0.037 & 0.071 \\
\hline 15 & REACLarge & C60ISO & 0.019 & 0.022 & 0.022 & 0.023 \\
\hline 16 & Intermol & CARBHB12 & 0.032 & 0.027 & 0.024 & 0.026 \\
\hline 17 & REACLarge & CDIE20 & 0.065 & 0.064 & 0.071 & 0.071 \\
\hline 18 & Intermol & CHB6 & 0.010 & 0.006 & 0.006 & 0.006 \\
\hline 19 & REACLarge & DARC & 0.009 & 0.009 & 0.007 & 0.008 \\
\hline 20 & Thermo & DC13 & 0.017 & 0.016 & 0.032 & 0.017 \\
\hline 21 & Thermo & DIPCS10 & 0.003 & 0.003 & 0.003 & 0.003 \\
\hline 22 & Thermo & FH51 & 0.043 & 0.045 & 0.045 & 0.045 \\
\hline 23 & Thermo & G21EA & 0.074 & 0.072 & 0.080 & 0.082 \\
\hline 24 & Thermo & G21IP & 0.012 & 0.012 & 0.013 & 0.013 \\
\hline 25 & Thermo & G2RC & 0.030 & 0.028 & 0.027 & 0.029 \\
\hline 26 & Intermol & HAL59 & 0.141 & 0.100 & 0.112 & 0.118 \\
\hline 27 & Intermol & HEAVY28 & 0.116 & 0.097 & 0.090 & 0.087 \\
\hline 28 & Thermo & HEAVYSB11 & 0.006 & 0.011 & 0.009 & 0.010 \\
\hline 29 & Conformer & ICONF & 0.019 & 0.021 & 0.033 & 0.025 \\
\hline 30 & Conformer & IDISP & 0.009 & 0.008 & 0.009 & 0.008 \\
\hline 31 & Intermol & IL16 & 0.003 & 0.002 & 0.002 & 0.001 \\
\hline 32 & Barrier & INV24 & 0.018 & 0.018 & 0.018 & 0.018 \\
\hline 33 & REACLarge & ISO34 & 0.032 & 0.033 & 0.032 & 0.034 \\
\hline 34 & REACLarge & ISOL24 & 0.045 & 0.045 & 0.044 & 0.047 \\
\hline 35 & REACLarge & MB16-43 & 0.027 & 0.051 & 0.062 & 0.062 \\
\hline 36 & Conformer & MCONF & 0.061 & 0.050 & 0.055 & 0.043 \\
\hline 37 & Thermo & NBPRC & 0.004 & 0.004 & 0.003 & 0.003 \\
\hline 38 & Thermo & PA26 & 0.009 & 0.007 & 0.008 & 0.008 \\
\hline 39 & REACLarge & PAREL & 0.060 & 0.061 & 0.061 & 0.062 \\
\hline 40 & Conformer & PCONF21 & 0.082 & 0.058 & 0.059 & 0.055 \\
\hline 41 & Intermol & PNICO23 & 0.040 & 0.020 & 0.017 & 0.022 \\
\hline 42 & Barrier & PX13 & 0.023 & 0.024 & 0.019 & 0.018 \\
\hline 43 & Thermo & RC21 & 0.032 & 0.033 & 0.034 & 0.029 \\
\hline 44 & Intermol & RG18 & 0.103 & 0.102 & 0.086 & 0.074 \\
\hline 45 & REACLarge & RSE43 & 0.169 & 0.176 & 0.167 & 0.108 \\
\hline 46 & Intermol & $\mathrm{S} 22$ & 0.020 & 0.015 & 0.016 & 0.012 \\
\hline 47 & Intermol & S66 & 0.067 & 0.073 & 0.071 & 0.082 \\
\hline 48 & Conformer & SCONF & 0.013 & 0.008 & 0.010 & 0.009 \\
\hline 49 & Thermo & SIE4X4 & 0.089 & 0.089 & 0.089 & 0.092 \\
\hline 50 & Thermo & TAUT15 & 0.092 & 0.097 & 0.098 & 0.105 \\
\hline 51 & Conformer & UPU23 & 0.069 & 0.069 & 0.072 & 0.067 \\
\hline 52 & Thermo & W4-11 & 0.042 & 0.042 & 0.044 & 0.042 \\
\hline 53 & Intermol & WATER27 & 0.007 & 0.008 & 0.007 & 0.014 \\
\hline 54 & Barrier & WCPT18 & 0.019 & 0.019 & 0.015 & 0.015 \\
\hline 55 & Thermo & YBDE18 & 0.011 & 0.010 & 0.011 & 0.011 \\
\hline \multicolumn{3}{|c|}{ Total WTMAD2 } & 2.429 & 2.332 & 2.307 & 2.229 \\
\hline
\end{tabular}


Table S18: Ranking of performance for WTMAD2 (kcal/mol) of the GMTKN55 dataset and its principal subsets. Heatmapping is from red (worst) via yellow to green (best). ${ }^{\text {(a) }}$

\begin{tabular}{|c|c|c|c|c|c|c|c|}
\hline Functional & $\begin{array}{l}\text { WTMAD2 } \\
\text { (kcal/mol) }\end{array}$ & Thermo & Barrier & REAClarge & Conformer & Intermol & $\mathbf{N C I}^{\mathrm{b}}$ \\
\hline LDA(SPW92) & 22.673 & 4.456 & 4.051 & 2.965 & 5.085 & 6.116 & 11.201 \\
\hline PBEsol-D3(BJ) & 14.277 & 2.912 & 3.108 & 2.253 & 3.120 & 2.884 & 6.004 \\
\hline PBE-D3(BJ) & 10.435 & 2.094 & 2.405 & 2.010 & 1.881 & 2.046 & 3.927 \\
\hline rPBE-D3(BJ) & 10.420 & 2.056 & 2.295 & 1.614 & 1.565 & 2.890 & 4.455 \\
\hline TPSS-D3(BJ) & 9.141 & 1.843 & 2.137 & 2.024 & 1.608 & 1.529 & 3.137 \\
\hline B97-D3(BJ) & 8.608 & 1.817 & 1.733 & 2.278 & 1.541 & 1.239 & 2.780 \\
\hline revTPSS-D3(BJ) & 8.424 & 1.941 & 2.036 & 1.771 & 1.328 & 1.348 & 2.676 \\
\hline revPBE-D3(BJ) & 8.340 & 1.774 & 2.044 & 1.708 & 1.545 & 1.270 & 2.815 \\
\hline SCAN-D3(BJ) & 7.947 & 1.671 & 1.950 & 1.303 & 1.297 & 1.726 & 3.023 \\
\hline M06-D3(0) & 7.748 & 1.146 & 0.640 & 1.456 & 2.978 & 1.528 & 4.506 \\
\hline SCANO & 7.685 & 1.640 & 1.033 & 1.317 & 1.707 & 1.989 & 3.696 \\
\hline CAM-QTP-02-D3(BJ) & 7.296 & 1.317 & 0.918 & 1.268 & 1.911 & 1.883 & 3.794 \\
\hline CAM-QTP-01-D3(BJ) & 6.809 & 1.256 & 0.936 & 1.207 & 1.808 & 1.602 & 3.410 \\
\hline CAM-QTP-00-D3(BJ) & 6.479 & 1.645 & 1.075 & 1.276 & 1.279 & 1.204 & 2.483 \\
\hline PBE0-D3(BJ) & 6.551 & 1.376 & 1.207 & 1.369 & 1.264 & 1.335 & 2.599 \\
\hline B3LYP-D3(BJ) & 6.502 & 1.314 & 1.141 & 1.662 & 1.147 & 1.238 & 2.385 \\
\hline M11 & 6.417 & 0.962 & 0.567 & 1.098 & 2.539 & 1.251 & 3.790 \\
\hline B97M-V & 6.365 & 1.196 & 1.003 & 1.559 & 1.759 & 0.848 & 2.607 \\
\hline SCAN0-D3(BJ) & 6.229 & 1.664 & 1.091 & 1.174 & 1.051 & 1.250 & 2.301 \\
\hline MN15-D3(BJ) & 5.772 & 0.999 & 0.519 & 1.017 & 2.126 & 1.111 & 3.237 \\
\hline revM11 & 5.732 & 1.123 & 0.761 & 1.284 & 1.610 & 0.954 & 2.564 \\
\hline PW6B95-D3(BJ) & 5.487 & 1.047 & 0.789 & 1.485 & 1.311 & 0.855 & 2.166 \\
\hline LC- $\omega$ PBEh-D3(BJ) & 5.489 & 1.324 & 0.949 & 1.237 & 1.134 & 0.844 & 1.978 \\
\hline revPBE0-D3(BJ) & 5.429 & 1.367 & 0.962 & 1.055 & 1.134 & 0.912 & 2.046 \\
\hline BH\&HLYP-D3(BJ) & 5.541 & 1.581 & 0.819 & 1.261 & 0.964 & 0.916 & 1.880 \\
\hline CAM-B3LYP-D3(BJ) & 5.323 & 1.129 & 0.883 & 1.261 & 1.236 & 0.814 & 2.050 \\
\hline revM06 & 5.301 & 1.013 & 0.523 & 1.151 & 1.673 & 0.940 & 2.613 \\
\hline M06-2X & 4.785 & 0.858 & 0.482 & 1.078 & 1.224 & 1.143 & 2.367 \\
\hline SCAN0-2 & 4.686 & 0.975 & 0.657 & 1.082 & 0.945 & 1.027 & 1.972 \\
\hline$\omega \mathrm{B} 97 \mathrm{X}-\mathrm{D} 3(\mathrm{BJ})$ & 4.686 & 0.975 & 0.657 & 1.082 & 0.945 & 1.027 & 1.972 \\
\hline$\omega \mathrm{B} 97 \mathrm{X}-\mathrm{V}$ & 3.959 & 1.020 & 0.561 & 1.070 & 0.730 & 0.578 & 1.308 \\
\hline$\omega \mathrm{B} 97 \mathrm{M}-\mathrm{D} 3(\mathrm{BJ})$ & 3.764 & 0.737 & 0.411 & 0.823 & 0.893 & 0.900 & 1.793 \\
\hline$\omega \mathrm{B} 97 \mathrm{M}-\mathrm{V}$ & 3.287 & 0.729 & 0.454 & 0.640 & 0.897 & 0.567 & 1.464 \\
\hline B2GP-PLYP-D3(BJ) & 3.187 & 0.626 & 0.415 & 0.662 & 0.635 & 0.850 & 1.485 \\
\hline DSD-PBE-D3(BJ) & 3.170 & 0.657 & 0.412 & 0.542 & 0.730 & 0.828 & 1.558 \\
\hline DSD-PBEP86-D3(BJ) & 3.099 & 0.551 & 0.448 & 0.486 & 0.649 & 0.966 & 1.615 \\
\hline noDispSD-SCAN69 & 2.983 & 0.582 & 0.524 & 0.671 & 0.475 & 0.731 & 1.206 \\
\hline rev $\omega \mathrm{B} 97 \mathrm{X}-2$ & 2.804 & 0.584 & 0.371 & 0.582 & 0.497 & 0.770 & 1.267 \\
\hline DSD-BLYP-D4 & 2.829 & 0.581 & 0.379 & 0.591 & 0.679 & 0.598 & 1.277 \\
\hline revDSD-PBEB95-D4 & 2.700 & 0.636 & 0.310 & 0.445 & 0.784 & 0.524 & 1.308 \\
\hline DSD-PBEP86-D4 & 2.649 & 0.538 & 0.365 & 0.631 & 0.554 & 0.562 & 1.116 \\
\hline DSD-PBEP86-NL & 2.639 & 0.583 & 0.395 & 0.569 & 0.535 & 0.558 & 1.093 \\
\hline DSD-PBE-D4 & 2.637 & 0.612 & 0.387 & 0.562 & 0.532 & 0.543 & 1.075 \\
\hline DSD-SCAN-D4 & 2.635 & 0.601 & 0.395 & 0.624 & 0.454 & 0.562 & 1.016 \\
\hline revDSD-BLYP-D4 & 2.592 & 0.568 & 0.341 & 0.576 & 0.483 & 0.624 & 1.107 \\
\hline revDSD-PBEP86-D3(BJ) & 2.422 & 0.535 & 0.310 & 0.551 & 0.458 & 0.568 & 1.026 \\
\hline revDSD-PBE-D4 & 2.462 & 0.646 & 0.354 & 0.531 & 0.433 & 0.497 & 0.930 \\
\hline revDSD-PBEP86-NL & 2.436 & 0.546 & 0.298 & 0.553 & 0.470 & 0.569 & 1.039 \\
\hline revDOD-PBEP86-D4 & 2.363 & 0.591 & 0.301 & 0.590 & $\underline{0.406}$ & $\underline{0.474}$ & $\underline{0.880}$ \\
\hline revDSD-PBEP86-D4 & 2.332 & 0.556 & 0.314 & 0.576 & $\overline{0.409}$ & 0.478 & $\overline{0.887}$ \\
\hline$\omega \mathrm{B} 97 \mathrm{M}(2)$ & 2.186 & 0.442 & 0.258 & 0.418 & 0.577 & 0.492 & 1.069 \\
\hline
\end{tabular}

(a) $\quad$ Bold underlined $=1^{\text {st }}$ place; bold $=2^{\text {nd }}$ place; italics $=3^{\text {rd }}$ place.

(b) $\mathrm{NCI}=$ Total Non-Covalent Interaction (Conformer/Intramolecular + Intermolecular) 


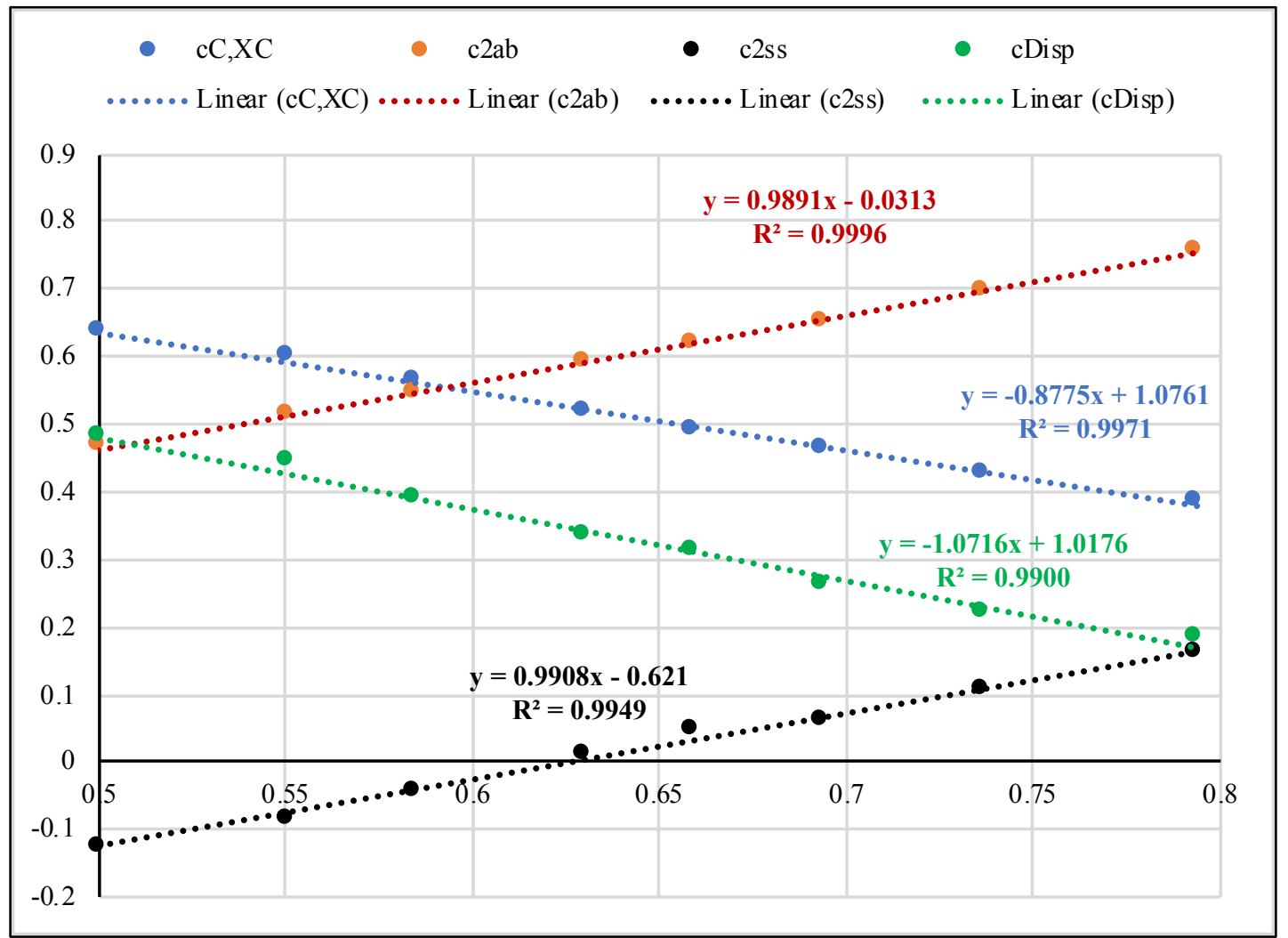

Figure S1: trends in the parameters $c_{2 a b}, c_{2 s s}, c_{D i s p}$, and $\mathrm{cC}, \mathrm{XC}$ for the DSD-SCANxD3(BJ) family as a function of the fraction $x$ of HF-like exchange. Note the remarkably linear behavior over the $x=0.5-0.8$ range, as well as the almost perfectly parallel $c_{2 a b}$ and c$_{2 s s}$ lines 


\section{Gaussian sample input files for CO}

\section{1. revDSD-BLYP-D3(BJ)}

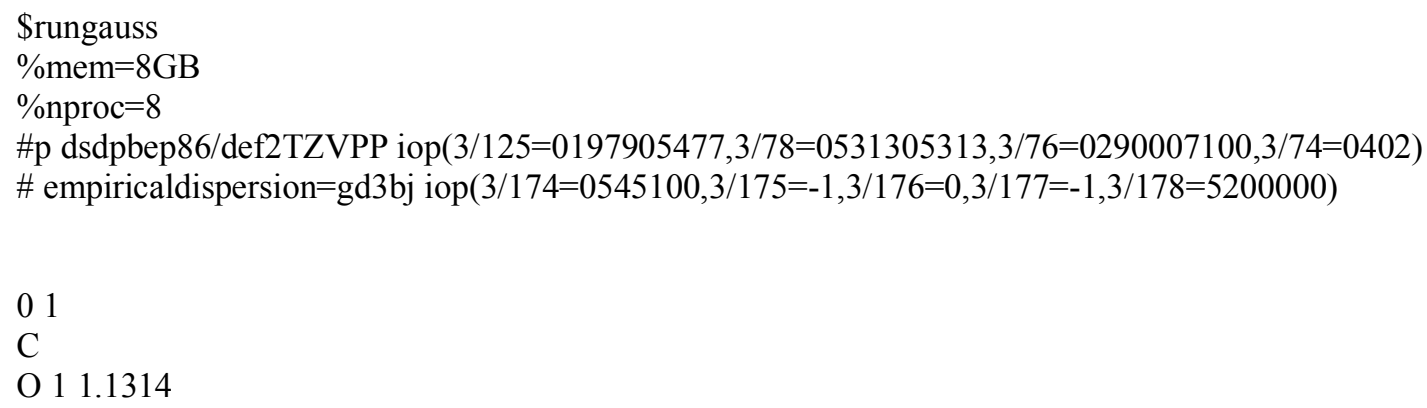

2. DSD-PBEP86-D3(BJ) (specified manually, for illustration)

\$rungauss

$\%$ mem $=8 \mathrm{~GB}$

$\%$ nproc $=8$

\#p dsdpbep86/def2TZVPP iop(3/125=0220005200,3/78 $=0440004400,3 / 76=0310006900,3 / 74=1004)$ \# empiricaldispersion $=\mathrm{gd} 3$ bj iop $(3 / 174=0480000,3 / 175=-1,3 / 176=0,3 / 177=-1,3 / 178=5600000)$

01

$\mathrm{C}$

O 11.1314

\section{3. revDSD-PBEP86-D3(BJ)}

\section{\$rungauss}

$\%$ mem $=8 \mathrm{~GB}$

$\%$ nproc $=8$

\#p dsdpbep86/def2TZVPP iop(3/125=0079905785,3/78=0429604296,3/76=0310006900,3/74=1004)

\# empiricaldispersion $=\mathrm{gd} 3$ bj iop $(3 / 174=0437700,3 / 175=-1,3 / 176=0,3 / 177=-1,3 / 178=5500000)$

01

$\mathrm{C}$

O 11.1314 


\section{ORCA sample input files for $\mathrm{CO}$}

\section{DOD-SCAN-D3(BJ)}

!PAL8

$\%$ MaxCore 2500

! RKS def2-TZVPP def2-tzvpp/c def2/j tightscf rijcosx GRID6 NoPOP d3bj

\%method

Exchange X_SCAN

Correlation C_SCAN

ScalHFX 0.66

ScalDFX 0.34

ScalGGAC 0.5048

ScalLDAC 0.5048

ScalMP2C 1.0

D3S6 0.3152

D3A2 5.75

D3S8 0.0

D3A1 0.0

end

\%mp2

DoSCS true

PS 0.6283

PT 0.0000

end

*xyz 01

$\begin{array}{llll}\mathrm{C} & 0.000000 & 0.000000 & 0.000000\end{array}$

O $\quad 0.000000 \quad 0.000000 \quad 1.131400$

2. noDispSD-SCAN $_{69}$

!PAL8

$\%$ MaxCore 2500

! RKS def2-TZVPP def2-tzvpp/c def2/j tightscf rijcosx GRID6 NoPOP

\%method

Exchange X_SCAN

Correlation $\bar{C}$ SCAN

ScalHFX 0.69

ScalDFX 0.31

ScalGGAC 0.4409

ScalLDAC 0.4409

ScalMP2C 1.0

end

\%mp2

DoSCS true

PS 0.6223

PT 0.2600

end 


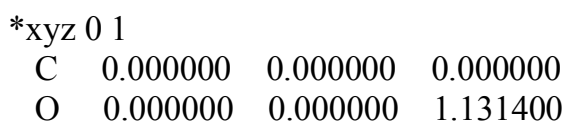

\section{3. revDSD-PBEP86-D3(BJ)}

!PAL8

\%MaxCore 2500

! RKS def2-TZVPP def2-tzvpp/c def2/j tightscf rijcosx GRID6 NoPOP d3bj

\%method

Exchange X_PBE

Correlation C_P86

ScalHFX 0.69

ScalDFX 0.31

ScalGGAC 0.4296

ScalLDAC 0.4296

ScalMP2C 1.0

D3S6 0.4377

D3A2 5.5

D3S8 0.0

D3A1 0.0

end

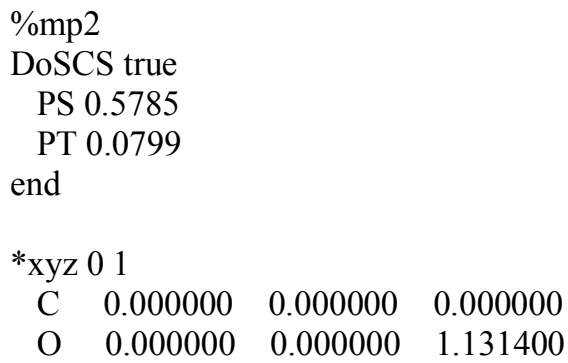

\section{4. revDSD-BLYP-D3(BJ)}

!PAL8

\%MaxCore 2500

! RKS def2-TZVPP def2-tzvpp/c def2/j tightscf rijcosx GRID6 NoPOP d3bj $\%$ method

Exchange X_B88

Correlation $\bar{C} \_$LYP

ScalHFX 0.71

ScalDFX 0.29

ScalGGAC 0.5313

ScalLDAC 0.5313

ScalMP2C 1.0

D3S6 0.5451

D3A2 5.20

D3S8 0.0

D3A1 0.0 


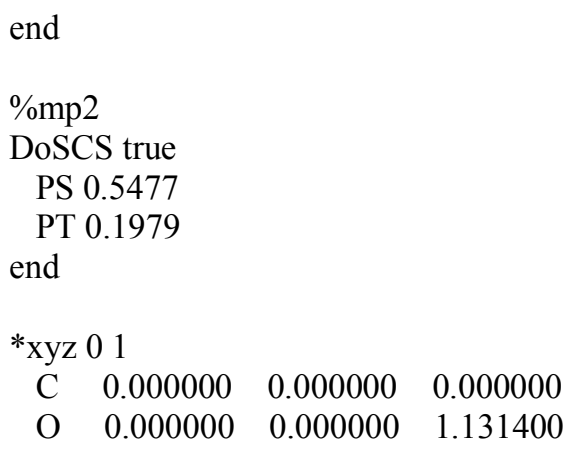

\section{5. revDOD-PBEP86-D3(BJ)}

!PAL8

$\%$ MaxCore 2500

! RKS def2-TZVPP def2-tzvpp/c def2/j tightscf rijcosx GRID6 NoPOP d3bj $\%$ method

Exchange X_PBE

Correlation $\overline{\mathrm{C}} \mathrm{P} 86$

ScalHFX 0.69

ScalDFX 0.31

ScalGGAC 0.4449

ScalLDAC 0.4449

ScalMP2C 1.0

D3S6 0.4770

D3A2 5.5

D3S8 0.0

D3A1 0.0

end

\%mp2

DoSCS true

PS 0.6055

PT 0.0000

end

*xyz 01

$\begin{array}{llll}\mathrm{C} & 0.000000 & 0.000000 & 0.000000\end{array}$

$\begin{array}{llll}\mathrm{O} & 0.000000 & 0.000000 & 1.131400\end{array}$ 


\section{References:}

(1) Gruzman, D.; Karton, A.; Martin, J. M. L. Performance of Ab Initio and Density Functional Methods for Conformational Equilibria of $\mathrm{C}(\mathrm{n}) \mathrm{H}(2 \mathrm{n}+2)$ Alkane Isomers $(\mathrm{n}=4-8)$. J. Phys. Chem. A 2009, 113 (43), 11974-11983. https://doi.org/10.1021/jp903640h.

(2) Grimme, S.; Antony, J.; Ehrlich, S.; Krieg, H. A Consistent and Accurate Ab Initio Parametrization of Density Functional Dispersion Correction (DFT-D) for the 94 Elements H-Pu. J. Chem. Phys. 2010, 132 (15), 154104. https://doi.org/10.1063/1.3382344.

(3) Lao, K. U.; Schäffer, R.; Jansen, G.; Herbert, J. M. Accurate Description of Intermolecular Interactions Involving Ions Using Symmetry-Adapted Perturbation Theory. J. Chem. Theory Comput. 2015, 11 (6), 2473-2486. https://doi.org/10.1021/ct5010593.

(4) Goerigk, L.; Hansen, A.; Bauer, C.; Ehrlich, S.; Najibi, A.; Grimme, S. A Look at the Density Functional Theory Zoo with the Advanced GMTKN55 Database for General Main Group Thermochemistry, Kinetics and Noncovalent Interactions. Phys. Chem. Chem. Phys. 2017, 19 (48), 32184-32215. https://doi.org/10.1039/C7CP04913G.

(5) Yu, H.; Truhlar, D. G. Components of the Bond Energy in Polar Diatomic Molecules, Radicals, and Ions Formed by Group-1 and Group-2 Metal Atoms. J. Chem. Theory Comput. 2015, 11 (7), 29682983. https://doi.org/10.1021/acs.jctc.5b00083.

(6) Kesharwani, M. K.; Karton, A.; Martin, J. M. L. Benchmark Ab Initio Conformational Energies for the Proteinogenic Amino Acids through Explicitly Correlated Methods. Assessment of Density Functional Methods. J. Chem. Theory Comput. 2016, 12 (1), 444-454. https://doi.org/10.1021/acs.jctc.5b01066.

(7) Goerigk, L.; Grimme, S. A General Database for Main Group Thermochemistry, Kinetics, and Noncovalent Interactions - Assessment of Common and Reparameterized ( Meta -)GGA Density Functionals. J. Chem. Theory Comput. 2010, 6 (1), 107-126. https://doi.org/10.1021/ct900489g.

(8) Zhao, Y.; Lynch, B. J.; Truhlar, D. G. Multi-Coefficient Extrapolated Density Functional Theory for Thermochemistry and Thermochemical Kinetics. Phys. Chem. Chem. Phys. 2005, 7 (1), 43. https://doi.org/10.1039/b416937a.

(9) Zhao, Y.; González-García, N.; Truhlar, D. G. Benchmark Database of Barrier Heights for Heavy Atom Transfer, Nucleophilic Substitution, Association, and Unimolecular Reactions and Its Use to Test Theoretical Methods. J. Phys. Chem. A 2005, 109 (9), 2012-2018. https://doi.org/10.1021/jp045141s.

(10) Guner, V.; Khuong, K. S.; Leach, A. G.; Lee, P. S.; Bartberger, M. D.; Houk, K. N. A Standard Set of Pericyclic Reactions of Hydrocarbons for the Benchmarking of Computational Methods: The Performance of Ab Initio, Density Functional, CASSCF, CASPT2, and CBS-QB3 Methods for the Prediction of Activation Barriers, Reaction Energetics, And. J. Phys. Chem. A 2003, 107 (51), 1144511459. https://doi.org/10.1021/jp035501w.

(11) Ess, D. H.; Houk, K. N. Activation Energies of Pericyclic Reactions: Performance of DFT, MP2, and CBS-QB3 Methods for the Prediction of Activation Barriers and Reaction Energetics of 1,3-Dipolar Cycloadditions, and Revised Activation Enthalpies for a Standard Set of Hydrocarbon . J. Phys. Chem. A 2005, 109 (42), 9542-9553. https://doi.org/10.1021/jp052504v.

(12) Dinadayalane, T. C.; Vijaya, R.; Smitha, A.; Sastry, G. N. Diels-Alder Reactivity of Butadiene and Cyclic Five-Membered Dienes ((CH) 4 X, X $=\mathrm{CH} 2$, SiH $2, \mathrm{O}, \mathrm{NH}, \mathrm{PH}$, and S) with Ethylene: A Benchmark Study. J. Phys. Chem. A 2002, 106 (8), 1627-1633. https://doi.org/10.1021/jp013910r.

(13) Steinmann, S. N.; Csonka, G.; Corminboeuf, C. Unified Inter- and Intramolecular Dispersion Correction Formula for Generalized Gradient Approximation Density Functional Theory. J. Chem. Theory Comput. 2009, 5 (11), 2950-2958. https://doi.org/10.1021/ct9002509.

(14) Krieg, H.; Grimme, S. Thermochemical Benchmarking of Hydrocarbon Bond Separation Reaction Energies: Jacob's Ladder Is Not Reversed! Mol. Phys. 2010, 108 (19-20), 2655-2666. https://doi.org/10.1080/00268976.2010.519729.

(15) Kozuch, S.; Bachrach, S. M.; Martin, J. M. L. Conformational Equilibria in Butane-1,4-Diol: A Benchmark of a Prototypical System with Strong Intramolecular H-Bonds. J. Phys. Chem. A 2014, 118 (1), 293-303. https://doi.org/10.1021/jp410723v.

(16) Sure, R.; Hansen, A.; Schwerdtfeger, P.; Grimme, S. Comprehensive Theoretical Study of All 1812 C60isomers. Phys. Chem. Chem. Phys. 2017, 19 (22), 14296-14305. https://doi.org/10.1039/c7cp00735c.

(17) Yu, L.-J.; Karton, A. Assessment of Theoretical Procedures for a Diverse Set of Isomerization Reactions Involving Double-Bond Migration in Conjugated Dienes. Chem. Phys. 2014, 441, 166-177. https://doi.org/10.1016/j.chemphys.2014.07.015.

(18) Johnson, E. R.; Mori-Sánchez, P.; Cohen, A. J.; Yang, W. Delocalization Errors in Density Functionals and Implications for Main-Group Thermochemistry. J. Chem. Phys. 2008, 129 (20), 204112. https://doi.org/10.1063/1.3021474.

(19) Zhao, Y.; Truhlar, D. G. The M06 Suite of Density Functionals for Main Group Thermochemistry, 
Thermochemical Kinetics, Noncovalent Interactions, Excited States, and Transition Elements: Two New Functionals and Systematic Testing of Four M06-Class Functionals and 12 Other Function. Theor. Chem. Acc. 2008, 120 (1-3), 215-241. https://doi.org/10.1007/s00214-007-0310-x.

(20) Grimme, S. Semiempirical Hybrid Density Functional with Perturbative Second-Order Correlation. $J$. Chem. Phys. 2006, 124 (3), 034108. https://doi.org/10.1063/1.2148954.

(21) Grimme, S.; Mück-Lichtenfeld, C.; Würthwein, E.-U.; Ehlers, A. W.; Goumans, T. P. M.; Lammertsma, K. Consistent Theoretical Description of 1,3-Dipolar Cycloaddition Reactions. J. Phys. Chem. A 2006, 110 (8), 2583-2586. https://doi.org/10.1021/jp057329x.

(22) Piacenza, M.; Grimme, S. Systematic Quantum Chemical Study of DNA-Base Tautomers. J. Comput. Chem. 2004, 25 (1), 83-99. https://doi.org/10.1002/jcc.10365.

(23) Woodcock, H. L.; Schaefer, H. F.; Schreiner, P. R. Problematic Energy Differences between Cumulenes and Poly-Ynes: Does This Point to a Systematic Improvement of Density Functional Theory? J. Phys. Chem. A 2002, 106 (49), 11923-11931. https://doi.org/10.1021/jp0212895.

(24) Schreiner, P. R.; Fokin, A. a; Pascal, R. a; de Meijere, A. Many Density Functional Theory Approaches Fail to Give Reliable Large Hydrocarbon Isomer Energy Differences. Org. Lett. 2006, 8 (17), 36353638. https://doi.org/10.1021/o10610486.

(25) Lepetit, C.; Chermette, H.; Gicquel, M.; Heully, J.; Chauvin, R. Description of Carbo -Oxocarbons and Assessment of Exchange-Correlation Functionals for the DFT Description of Carbo -Mers. J. Phys. Chem. A 2007, 111 (1), 136-149. https://doi.org/10.1021/jp064066d.

(26) Lee, J. S. Accurate Ab Initio Binding Energies of Alkaline Earth Metal Clusters. J. Phys. Chem. A 2005, 109 (51), 11927-11932. https://doi.org/10.1021/jp040705d.

(27) Karton, A.; Martin, J. M. L. Explicitly Correlated Benchmark Calculations on C 8 H 8 Isomer Energy Separations: How Accurate Are DFT, Double-Hybrid, and Composite Ab Initio Procedures? Mol. Phys. 2012, 110 (19-20), 2477-2491. https://doi.org/10.1080/00268976.2012.698316.

(28) Zhao, Y.; Tishchenko, O.; Gour, J. R.; Li, W.; Lutz, J. J.; Piecuch, P.; Truhlar, D. G. Thermochemical Kinetics for Multireference Systems: Addition Reactions of Ozone. J. Phys. Chem. A 2009, 113 (19), 5786-5799. https://doi.org/10.1021/jp811054n.

(29) Manna, D.; Martin, J. M. L. What Are the Ground State Structures of C 20 and C 24 ? An Explicitly Correlated Ab Initio Approach. J. Phys. Chem. A 2016, 120 (1), 153-160.

https://doi.org/10.1021/acs.jpca.5b10266.

(30) Friedrich, J.; Hänchen, J. Incremental CCSD(T)(F12*)|MP2 - A Black Box Method to Obtain Highly Accurate Reaction Energies. J. Chem. Theory Comput. 2013, 131118164711009. https://doi.org/10.1021/ct4008074.

(31) Friedrich, J. Efficient Calculation of Accurate Reaction Energies - Assessment of Different Models in Electronic Structure Theory. J. Chem. Theory Comput. 2015, 11 (8), 3596-3609. https://doi.org/10.1021/acs.jctc.5b00087.

(32) Curtiss, L. A.; Raghavachari, K.; Trucks, G. W.; Pople, J. A. Gaussian-2 Theory for Molecular Energies of First- and Second-row Compounds. J. Chem. Phys. 1991, 94 (11), 7221-7230. https://doi.org/10.1063/1.460205.

(33) Curtiss, L. A.; Raghavachari, K.; Redfern, P. C.; Pople, J. A. Assessment of Gaussian-2 and Density Functional Theories for the Computation of Enthalpies of Formation. J. Chem. Phys. 1997, 106 (3), 1063-1079. https://doi.org/10.1063/1.473182.

(34) Kozuch, S.; Martin, J. M. L. Halogen Bonds: Benchmarks and Theoretical Analysis. J. Chem. Theory Comput. 2013, 9, 1918-1931. https://doi.org/10.1021/ct301064t.

(35) Rezáč, J.; Riley, K. E.; Hobza, P. Benchmark Calculations of Noncovalent Interactions of Halogenated Molecules. J. Chem. Theory Comput. 2012, 8 (11), 4285-4292. https://doi.org/10.1021/ct300647k.

(36) Schwabe, T.; Grimme, S. Double-Hybrid Density Functionals with Long-Range Dispersion Corrections: Higher Accuracy and Extended Applicability. Phys. Chem. Chem. Phys. 2007, 9 (26), 3397-3406. https://doi.org/10.1039/b704725h.

(37) Grimme, S. Seemingly Simple Stereoelectronic Effects in Alkane Isomers and the Implications for Kohn-Sham Density Functional Theory. Angew. Chemie Int. Ed. 2006, 45 (27), 4460-4464. https://doi.org/10.1002/anie.200600448.

(38) Goerigk, L.; Grimme, S. Efficient and Accurate Double-Hybrid-Meta-GGA Density FunctionalsEvaluation with the Extended GMTKN30 Database for General Main Group Thermochemistry, Kinetics, and Noncovalent Interactions. J. Chem. Theory Comput. 2011, 7 (2), 291-309. https://doi.org/10.1021/ct100466k.

(39) Grimme, S.; Steinmetz, M.; Korth, M. How to Compute Isomerization Energies of Organic Molecules with Quantum Chemical Methods. J. Org. Chem. 2007, 72 (6), 2118-2126.

https://doi.org/10.1021/jo062446p.

(40) Goerigk, L.; Sharma, R. The INV24 Test Set: How Well Do Quantum-Chemical Methods Describe 
Inversion and Racemization Barriers? Can. J. Chem. 2016, 11 (August), cjc-2016-0290. https://doi.org/10.1139/cjc-2016-0290.

(41) Huenerbein, R.; Schirmer, B.; Moellmann, J.; Grimme, S. Effects of London Dispersion on the Isomerization Reactions of Large Organic Molecules: A Density Functional Benchmark Study. Phys. Chem. Chem. Phys. 2010, 12 (26), 6940. https://doi.org/10.1039/c003951a.

(42) Fogueri, U. R.; Kozuch, S.; Karton, A.; Martin, J. M. L. The Melatonin Conformer Space: Benchmark and Assessment of Wave Function and DFT Methods for a Paradigmatic Biological and Pharmacological Molecule. J. Phys. Chem. A 2013, 117 (10), 2269-2277.

https://doi.org/10.1021/jp312644t.

(43) Grimme, S.; Kruse, H.; Goerigk, L.; Erker, G. The Mechanism of Dihydrogen Activation by Frustrated Lewis Pairs Revisited. Angew. Chemie Int. Ed. 2010, 49 (8), 1402-1405. https://doi.org/10.1002/anie.200905484.

(44) Setiawan, D.; Kraka, E.; Cremer, D. Strength of the Pnicogen Bond in Complexes Involving Group Va Elements N, P, and As. J. Phys. Chem. A 2015, 119 (9), 1642-1656. https://doi.org/10.1021/jp508270g.

(45) Karton, A.; O’Reilly, R. J.; Chan, B.; Radom, L. Determination of Barrier Heights for Proton Exchange in Small Water, Ammonia, and Hydrogen Fluoride Clusters with G4(MP2)-Type, MP n , and SCS-MP n Procedures-A Caveat. J. Chem. Theory Comput. 2012, 8 (9), 3128-3136. https://doi.org/10.1021/ct3004723.

(46) Neese, F.; Schwabe, T.; Kossmann, S.; Schirmer, B.; Grimme, S. Assessment of Orbital-Optimized, Spin-Component Scaled Second-Order Many-Body Perturbation Theory for Thermochemistry and Kinetics. J. Chem. Theory Comput. 2009, 5, 3060-3073.

(47) Jurečka, P.; Šponer, J.; Černý, J.; Hobza, P. Benchmark Database of Accurate (MP2 and CCSD(T) Complete Basis Set Limit) Interaction Energies of Small Model Complexes, DNA Base Pairs, and Amino Acid Pairs. Phys. Chem. Chem. Phys. 2006, 8 (17), 1985-1993.

https://doi.org/10.1039/B600027D.

(48) Rezáč, J.; Riley, K. E.; Hobza, P. S66: A Well-Balanced Database of Benchmark Interaction Energies Relevant to Biomolecular Structures. J. Chem. Theory Comput. 2011, 7 (8), 2427-2438. https://doi.org/10.1021/ct2002946.

(49) Csonka, G. I.; French, A. D.; Johnson, G. P.; Stortz, C. A. Evaluation of Density Functionals and Basis Sets for Carbohydrates. J. Chem. Theory Comput. 2009, 5 (4), 679-692. https://doi.org/10.1021/ct8004479.

(50) Karton, A.; Rabinovich, E.; Martin, J. M. L.; Ruscic, B. W4 Theory for Computational Thermochemistry: In Pursuit of Confident Sub-KJ/Mol Predictions. J. Chem. Phys. 2006, 125 (14), 144108. https://doi.org/10.1063/1.2348881.

(51) Kruse, H.; Mladek, A.; Gkionis, K.; Hansen, A.; Grimme, S.; Sponer, J. Quantum Chemical Benchmark Study on 46 RNA Backbone Families Using a Dinucleotide Unit. J. Chem. Theory Comput. 2015, 11 (10), 4972-4991. https://doi.org/10.1021/acs.jctc.5b00515.

(52) Karton, A.; Daon, S.; Martin, J. M. L. W4-11: A High-Confidence Benchmark Dataset for Computational Thermochemistry Derived from First-Principles W4 Data. Chem. Phys. Lett. 2011, 510, 165-178. https://doi.org/10.1016/j.cplett.2011.05.007.

(53) Bryantsev, V.; Diallo, M.; van Duin, A.; Goddard, W. Evaluation of B3LYP, X3LYP, and M06-Class Density Functionals for Predicting the Binding Energies of Neutral, Protonated, and Deprotonated Water Clusters. J. Chem. Theory Comput. 2009, 5 (4), 1016-1026.

(54) Karton, A.; O’Reilly, R. J.; Radom, L. Assessment of Theoretical Procedures for Calculating Barrier Heights for a Diverse Set of Water-Catalyzed Proton-Transfer Reactions. J. Phys. Chem. A 2012, 116 (16). https://doi.org/10.1021/jp301499y.

(55) Zhao, Y.; Ng, H. T.; Peverati, R.; Truhlar, D. G. Benchmark Database for Ylidic Bond Dissociation Energies and Its Use for Assessments of Electronic Structure Methods. J. Chem. Theory Comput. 2012 , 8 (8), 2824-2834. https://doi.org/10.1021/ct300457c.

(56) Geary, R. C. The Ratio of the Mean Deviation to the Standard Deviation as a Test of Normality. Biometrika 1935, 27 (3-4), 310-332. https://doi.org/10.1093/biomet/27.3-4.310.

(57) Geary, R. C. Moments of the Ratio of the Mean Deviation to the Standard Deviation for Normal Samples. Biometrika 1936, 28 (3-4), 295-307. https://doi.org/10.1093/biomet/28.3-4.295. 
